GEOLOGICAL SURVEY CIRCULAR 262

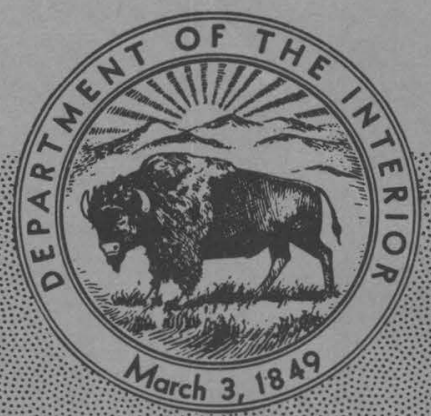

STRATIGRAPHIC SECTIONS OF THE PHOSPHORIA FORMATION IN

IDAHO, 1947-48, PART 3 

UNITED STATES DEPARTMENT OF THE INTERIOR

Douglas McKay, Secretary

GEOLOGICAL SURVEY

W. E. Wrather, Director

GEOLOGICAL SURVEY CIRCULAR 262

\section{STRATIGRAPHIC SECTIONS OF THE PHOSPHORIA FORMATION IN IDAHO, 1947-48, PART 3}

By F. W. O'Malley, D. F. Davidson, R. A. Hoppin, and R. P. Sheldon

This report concerns work done partly on behalf of the

U. S. Atomic Energy Commission

and is published with the permission of the Commission.

Washington, D. C... 1953

Free on application to the Geological Survey, Washington 25, D. C. 


\title{
STRATIGRAPHIC SECTIONS OF THE PHOSPHORIA FORMATION IN IDAHO, 1947-48, PART 3
}

\author{
By F. W. O'Malley, D. F. Davidson, R. A. Hoppin, and R. P. Sheldon
}

\section{CONTENTS}

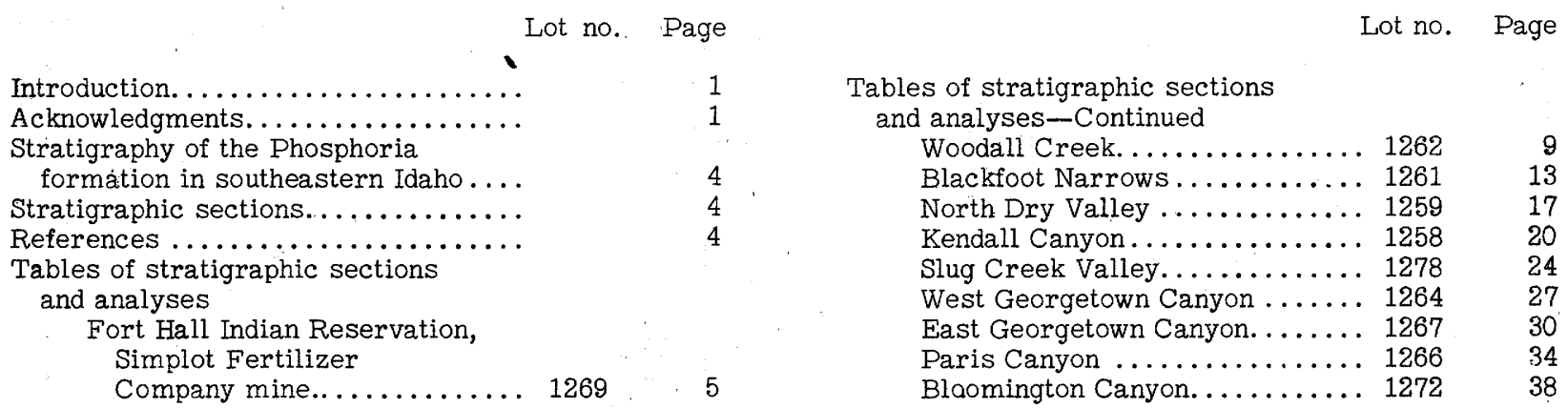

ILLUSTRATIONS

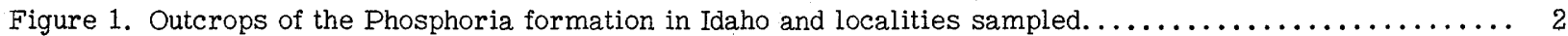

2. Generalized section of the Phosphoria formation at Trail Canyon (lot no. 1206) .............. 3

\section{INTRODUCTION}

The U. S. Geological Survey has measured and sampled the Phosphoria formation at many localities in Idaho and other western states. These data will not be fully synthesized and analyzed for several years, but segments of the data, accompanied by little or no interpretation, are published as preliminary reports as they are assembled. This report, which contains abstracts of many of the sections in southeastern Idahu (fig. 1), is one of this series and is the third report of data gathered in Idaho during 1947 and 1948. The field and laboratory procedures adopted in these investigations are described rather fully in a companion report (McKelvey and others, 1953).

Many people have taken part in this investigation, which was organized and supervised by V. E. McKelvey. D. A. Bostwick, R. M. Campbell, R. A. Gulbrandsen, R. A. Harris, R. L. Parker, R. A. Smart, J. E. Smedley, R. H. Thurston, and R. G. Waring participated in the description of strata and the collection of samples referred to in this report. D. B. Dimick, Jack George, W. S. Hunziker, J. E. Jones, H. A. Larsen, and T. K. Rigby assisted in the preparation of trenches and the collection, crushing, and splitting of samples in the field. The laboratory preparation of samples for chemical analysis was done in Denver, Colo., under the direction of W. P. Huleatt.

All the $\mathrm{P}_{2} \mathrm{O}_{5}, \mathrm{~F}$, and acid-insoluble analyses were made for the Survey by the U. S. Burea of Mines at the Northwest Electrodevelopment Laboratory, Albany, Oreg., under the direction of S. M. Shelton and M. L. Wright. The $\mathrm{Al}_{2} \mathrm{O}_{3}, \mathrm{Fe}_{2} \mathrm{O}_{3}$, and loss-on-ignition analyses were made in the Trace Elements Section laboratory of the Survey in Washington, D. C., under the direction of J. C. Rabbitt by chemists I. Barlow, A. B. Caemmerer, F. Cuttitta, J. L. Greene, N. Guttag, C. Hoy, E. H. Pendergrass, J. J. Rowe, anci $A$. L. White.

Compilation of the data has been largely by R. P. Sheldon and F. D. Frieske under the supervision of R. W. Swanson. Organization of the tabular data has been largely by Anita Cozzetto.

\section{ACKNOWLEDGMENTS}

Special thanks are due W. W. Rubey, J. Steele Williams, and A. E. Weissenborn who have given much advice in planning; and organizing the field program.

The cost of both the field and laboratory investigations has been partly borne by the Division of Raw Materials of the Atomic Energy Commission.

It is a pleasure to acknowledge the fine cooperation extended to the field party by the local residents, property owners, and operating phosphate companies, who furnished information and services and gave access to property. A. J. Winters, Superintendent of the Montpelier schools; E. M. Norris, C. T. Russell, and L. E. Traeger of the Anaconda Copper Miring Co. ; 


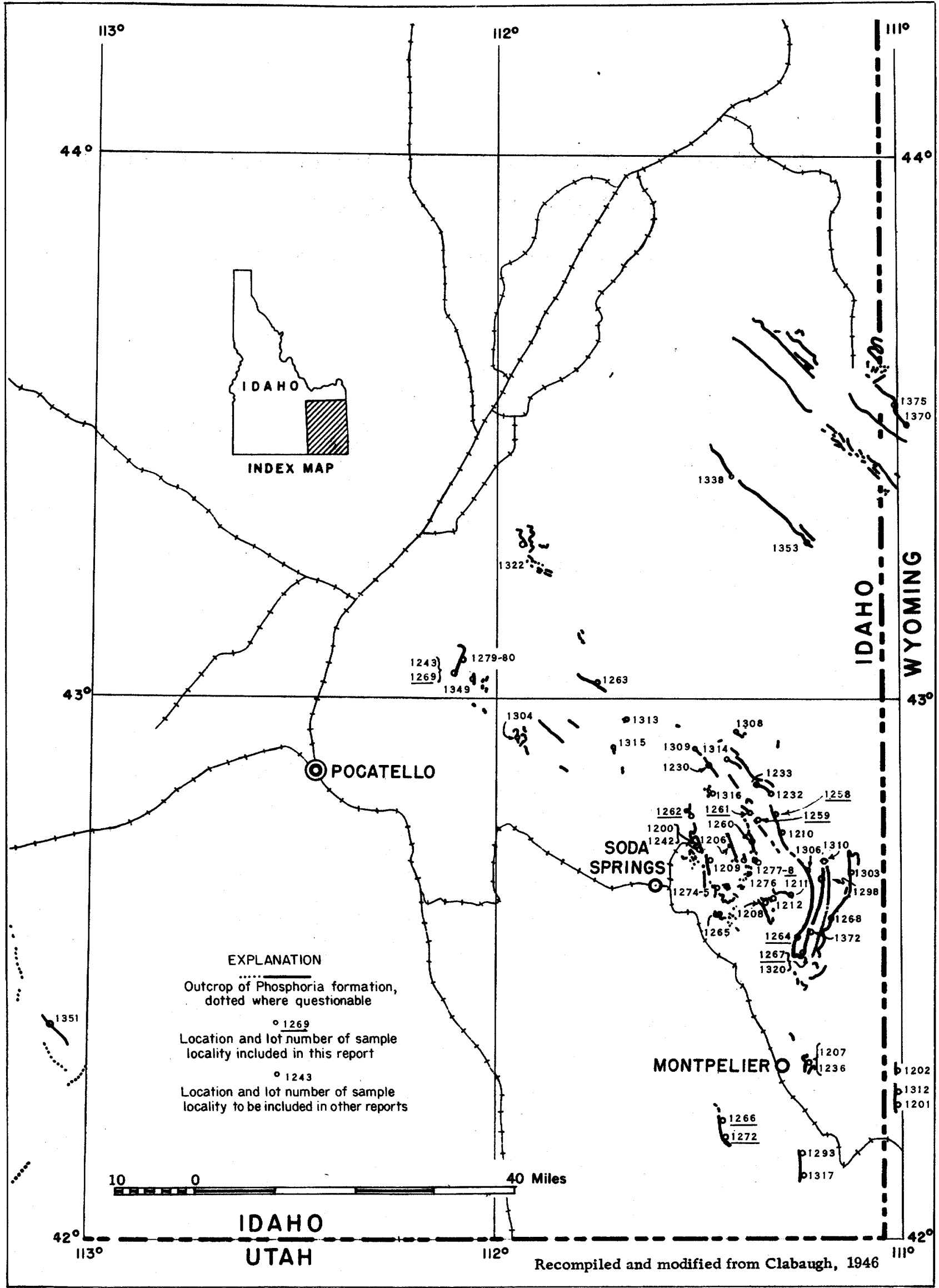

Figure 1.-Outcrops of the Phosphoria formation in Idaho and localities sampled. 


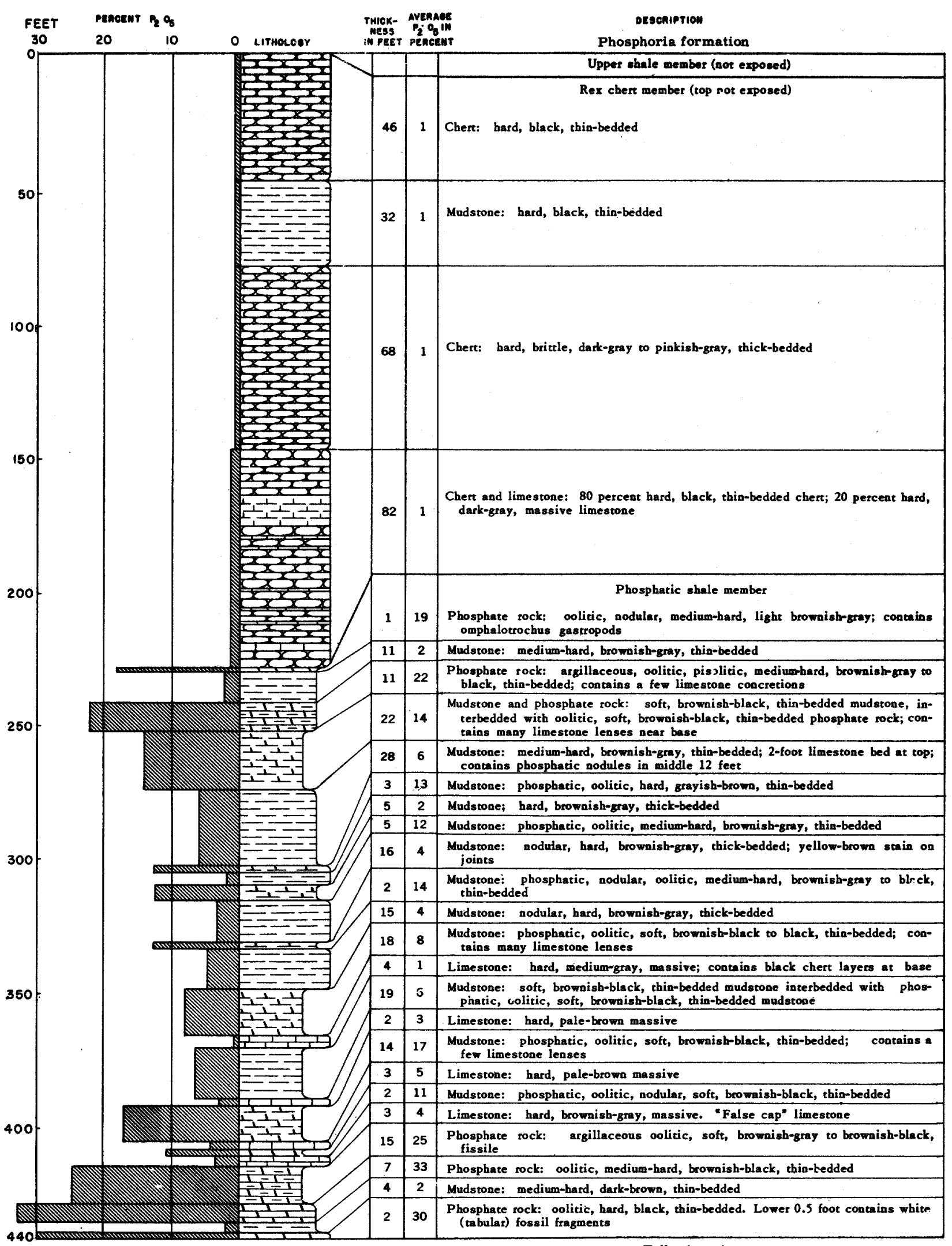

Wells formation

Figure 2.-Generalized section of the Phosphoria formation at Trail Canyon (lot no. 1206). 
D. L. King of the San Francisco Chemical Co.; and G. A. McHugh and H. B. Fowler of the Simplot Fertilizer Co. have been especially helpful in this connection.

\section{STRATIGRAPHY OF THE PHOSPHORIA FORMATION IN SOUTHEASTERN IDAHO}

At its type locality in southeastern Idaho (Richards and Mansfield, 1912), the Phosphoria formation consists of a lower member, the phosphatic shale, about 180 feet thick and an upper member, the Rex chert, about 240 feet thick; in most of southeastern Idaho and western Wyoming, however, another member, a thin-bedded cherty mudstone 15 to 75 feet thick, overlies the Rex chert member, though it is not well defined at the type locality.

The Phosphoria formation overlies the Wells formation of Pennsylvanian age and underlies the Dinwoody formation of Triassic age. The upper 50 to 75 feet of the Wells formation consists of gray fossiliferous cherty limestone that contains some thin phosphatic layers. It may be the correlative of the lowermost member (A member) of the Phosphoria formation in Montana and the lower limestone member of the Park City formation in Utah (McKelvey, 1949).

In southeastern Idaho most of the phosphatic beds are in the phosphatic shale member, and it is on this member that most of our studies have been focused. It consists of many thin layers, some of which persist over the whole area. They may be grouped into several broad units, as yet unnamed, as shown on figure 2 .

\section{STRATIGRAPHIC SECTIONS}

Abstracts of stratigraphic sections measured at 10 localities, and the available analytical data, are presented in the following pages. Their locations, as well as the locations of the sections reported (McKelvey, and others, 1953a, b) previously and of others to be reported later, are shown on figure 1.

\section{REFERENCES}

Clabaugh, P. S., 1946, Permian phosphate deposits of Montana, Idaho, Wyoming, and Utah: U. S. Geol. Survey, Strategic Minerals Investigations Preliminary Map 3-198.

McKelvey, V. E., 1949, Geological studies of the western phosphate field: Am. Inst. Min. Met. Eng. Mining Trans. , v. 184, p. 270-279.

McKelvey, V. E., Davidson, D. F., O'Malley, F. W., and Smith, L. E., 1953a, Stratigraphic sections of the Phosphoria formation in Idaho, 1947-48, part I: U. S. Geol. Survey Circ. 208.

, Armstrong, F. C., Gulbrandsen, R. A., and Campbell, R. M., 1953b, Stratigraphic sections of Phosphoria formation in Idaho, 1947-48, part II: U. S. Geol. Survey Circ. 261.

Richards, R. W., and Mansfield, G. R., 1912, The Bannock overthrust: a major fault in southeastern Idaho and northeastern Utah: Jour. Geology, v. 20, p. 684 . 
FORT HALL INDIAN RESERVATION, SIMPLOT FERTILIZER COMPANY MINE, IDAHO. LOT NO. 1269.

Lower part of phosphatic shale member of Phosphoria formation, weathered and unweathered sections, sampled in pits at Simplot mine, Fort

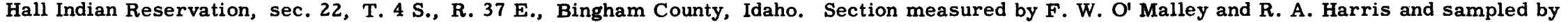

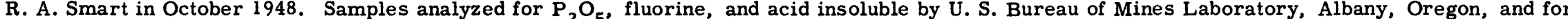
other constituents by Trace Elements Section Laboratory, U. S. Geological Survey, Washington, D. C.

\begin{tabular}{|c|c|c|c|c|c|c|c|c|c|c|c|}
\hline \multirow{2}{*}{$\begin{array}{c}\text { Bed } \\
\text { no. }\end{array}$} & \multirow[b]{2}{*}{ Rock description } & \multirow{2}{*}{$\begin{array}{c}\text { Sample } \\
\text { no. }\end{array}$} & \multirow{2}{*}{$\begin{array}{c}\text { Thichness } \\
\text { (feet) }\end{array}$} & \multicolumn{6}{|c|}{ Chemical analyses (percent) } & \multirow{2}{*}{$\begin{array}{c}\text { Cumulative } \\
\text { thickness } \\
\text { (feet) }\end{array}$} & \multirow{2}{*}{$\begin{array}{l}\text { Thickness } \mathrm{x} \\
\text { percent } \mathrm{P}_{2} \mathrm{O}_{5} \\
\text { (cumulative) }\end{array}$} \\
\hline & & & & $\mathrm{P}_{2} \mathrm{O}_{5}$ & $\mathrm{Al}_{2} \mathrm{O}_{3}$ & $\mathrm{Fe}_{2} \mathrm{O}_{3}$ & $F$ & $\begin{array}{l}\text { Loss on } \\
\text { ignition }\end{array}$ & $\begin{array}{c}\text { Acid } \\
\text { insoluble }\end{array}$ & & \\
\hline
\end{tabular}

Phosphatic shale member of Phosphoria formation

Hard, unaltered section

or P-44 $\quad$ Mudstone, phosphatic

P-43 Phosphate rock, argillaceous

P-42 Phosphate rock, argillaceous

P-41 Phosphate rock, argillaceous

P-40 Mudstone, phosphatic

P-39 Phosphate rock, argillaceous

P-38 Mudstone, phosphatic

P-37 Limestone, argillaceous

P-36 Mudstone, phosphatic

-- Limestone concretion

P-35 Phosphate rock, argillaceous, calcareous

\section{Pit C}

\begin{tabular}{|l|}
$2721-$ WOM \\
$2720-$ WOM \\
$2718-$ WOM \\
$2717-$-WOM \\
$2716-$ WOM \\
$2715-$ WOM \\
$2714-$ WOM \\
$2719-$ WOM \\
$2713-$ WOM \\
$2712-$ WOM \\
$2711-$ WOM \\
$2710-$ WOM \\
$2709-$ WOM \\
$2708-$ WOM \\
$2706-$ WOM \\
$2707-$ WOM \\
$2705-$ WOM
\end{tabular}

1.5
0.8
10.0
0.6
1.8
0.9
1.6
0.9
1.1
0.8
1.1
1.0
1.2
1.6
3.0
$(1.2)$
4.2

--
--
--
--
--
--
--
--
--
--
--
--
--
--
--
--
--

--
--
--
--
--
--
--
--
--
--
--
--
--
--
--
--
--

83.2
13.5
85.5
71.6
75.1
44.4
61.3
33.0
21.8
43.7
46.8
38.8
44.9
23.4
3.4
44.8
31.2

2. 25

26.89

32.89

35.47

44.65

59.68

72.32

90.05

90.05
117.55

131.55

149.37

166.37

185.21

187.77

191.97

$--$

267. 15

About 10.0 feet of section not logged and sampled between bottom of pit C section and top of pit B section; rocks in pit B are stratigraphically below those measured in pit $C$, but exact correlation is not possible.

P-34 Phosphate rock, argillaceous

P-33 Limestone

P-32 Limestone, phosphatic. argillaceous

P-31 Mudstone, calcareous, phosphatic

$2275-W O M$
$2274-W O M$
$2273-W O M$
$2272-W O M$

Pit B

\begin{tabular}{l|r|r|r|r|}
1.5 & 24.7 & -- & -- & - \\
1.5 & 3.5 & -- & -- & - \\
1.7 & 13.3 & -- & -- & -- \\
0.4 & 9.4 & -- & -- & --
\end{tabular}

--
--
-

\begin{tabular}{r|}
22.8 \\
4.5 \\
25.1 \\
39.7
\end{tabular}

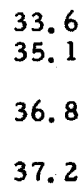




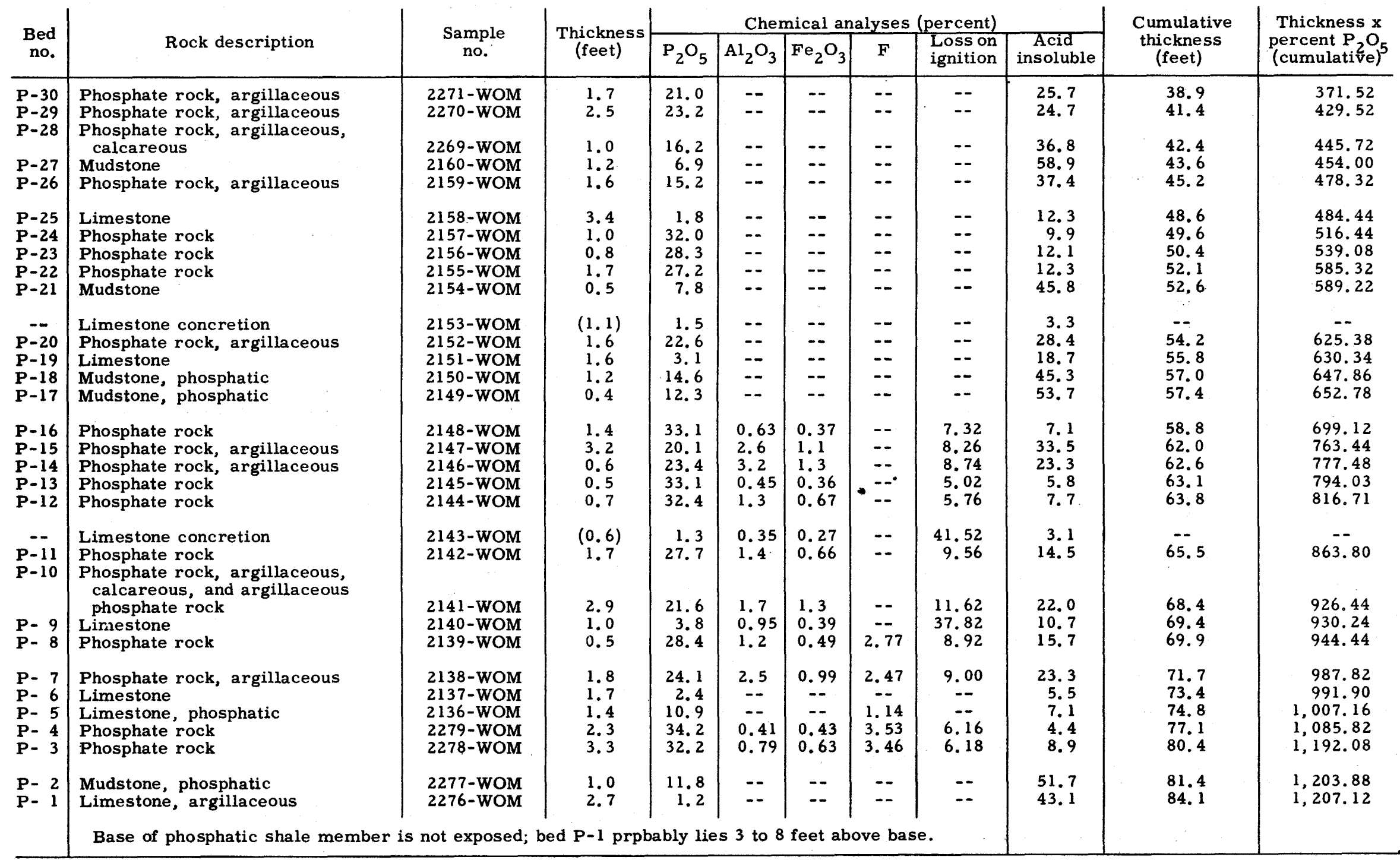




\begin{tabular}{|c|c|c|c|c|c|c|c|c|c|c|c|}
\hline \multicolumn{12}{|c|}{ Pit E } \\
\hline $\begin{array}{l}P-51 \\
P-50 \\
P-49 \\
P-48 \\
P-47\end{array}$ & $\begin{array}{l}\text { Phosphate rock } \\
\text { Phosphate rock, argillaceous } \\
\text { Phosphate rock, argillaceous } \\
\text { Phosphate rock, argillaceous } \\
\text { Mudstone, phosphatic }\end{array}$ & $\begin{array}{l}\text { 2704-WOM } \\
2703-\text { WOM } \\
2702-\text { WOM } \\
2701-\text { WOM } \\
2700-\text { WOM }\end{array}$ & $\begin{array}{l}1.2 \\
0.6 \\
0.7 \\
0.5 \\
0.3\end{array}$ & $\begin{array}{l}31.3 \\
26.4 \\
23.2 \\
27.1 \\
7.9\end{array}$ & $\begin{array}{l}-- \\
-- \\
-- \\
-\end{array}$ & $\begin{array}{l}-- \\
-- \\
-- \\
--\end{array}$ & $\begin{array}{l}-- \\
-- \\
-- \\
--\end{array}$ & $\begin{array}{l}-- \\
- \\
-- \\
--\end{array}$ & $\begin{array}{l}12.7 \\
22.0 \\
31.0 \\
22.2 \\
53.7\end{array}$ & $\begin{array}{l}1.2 \\
1.8 \\
2.5 \\
3.0 \\
3.3\end{array}$ & $\begin{array}{l}37.56 \\
53.40 \\
69.64 \\
83.19 \\
85.56\end{array}$ \\
\hline $\begin{array}{l}\text { P-46 } \\
P-45 \\
P-44 \\
P-43 \\
P-42\end{array}$ & $\begin{array}{l}\text { Phosphate rock, argillaceous } \\
\text { Phosphate rock, argillaceous } \\
\text { Phosphate rock, argillaceous } \\
\text { Mudstone, phosphatic } \\
\text { Phosphate rock, argillaceous }\end{array}$ & $\begin{array}{l}\text { 2699-WOM } \\
2698-\text { WOM } \\
2697-\text { WOM } \\
2696-\text { WOM } \\
2695-\text { WOM }\end{array}$ & $\begin{array}{l}0.6 \\
1.0 \\
0.5 \\
0.7 \\
1.1\end{array}$ & $\begin{array}{l}23.9 \\
23.7 \\
19.6 \\
13.1 \\
21.3\end{array}$ & $\begin{array}{l}-- \\
-- \\
-- \\
--\end{array}$ & $\begin{array}{l}-- \\
-- \\
-- \\
--\end{array}$ & $\begin{array}{l}-- \\
-- \\
-- \\
--\end{array}$ & $\begin{array}{l}-- \\
-- \\
-- \\
--\end{array}$ & $\begin{array}{l}26.4 \\
27.9 \\
58.7 \\
55.8 \\
36.1\end{array}$ & $\begin{array}{l}3.9 \\
4.9 \\
5.4 \\
6.1 \\
7.2\end{array}$ & $\begin{array}{r}99.90 \\
123.60 \\
133.40 \\
142.57 \\
166.00\end{array}$ \\
\hline $\begin{array}{l}P-41 \\
P-40 \\
P-39 \\
P-38 \\
P-37\end{array}$ & $\begin{array}{l}\text { Mudstone, phosphatic } \\
\text { Phosphate rock, argillaceous } \\
\text { Mudstone, phosphatic } \\
\text { Phosphate rock, argillaceous } \\
\text { Limestone }\end{array}$ & $\begin{array}{l}\text { 2694-WOM } \\
2693-\text { WOM } \\
2692-\text { WOM } \\
2691-\text { WOM } \\
2690-\text { WOM }\end{array}$ & $\begin{array}{l}0.6 \\
1.4 \\
1.1 \\
0.5 \\
2.8\end{array}$ & $\begin{array}{r}17.6 \\
17.9 \\
8.6 \\
25.6 \\
5.6\end{array}$ & $\begin{array}{l}-- \\
-- \\
-- \\
--\end{array}$ & $\begin{array}{l}-- \\
-- \\
-- \\
--\end{array}$ & $\begin{array}{l}-- \\
-- \\
-- \\
--\end{array}$ & $\begin{array}{l}-- \\
-- \\
-- \\
--\end{array}$ & $\begin{array}{r}46.2 \\
40.6 \\
58.8 \\
27.2 \\
8.9\end{array}$ & $\begin{aligned} 7.8 \\
9.2 \\
10.3 \\
10.8 \\
13.6\end{aligned}$ & $\begin{array}{l}176.56 \\
201.62 \\
211.08 \\
223.88 \\
239.56\end{array}$ \\
\hline $\begin{array}{l}P-36 \\
P-35 \\
P-34 \\
P-33 \\
P-32\end{array}$ & $\begin{array}{l}\text { Phosphate rock } \\
\text { Phosphate rock, argillaceous } \\
\text { Limestone } \\
\text { Phosphate rock } \\
\text { Phosphate rock }\end{array}$ & $\begin{array}{l}\text { 2689-WOM } \\
2688-\text { WOM } \\
2687-\text { WOM } \\
2686-\text { WOM } \\
2685-\text { WOM }\end{array}$ & $\begin{array}{l}0.5 \\
0.6 \\
1.6 \\
0.6 \\
0.8\end{array}$ & $\begin{array}{l}26.8 \\
21.7 \\
1.7 \\
34.3 \\
30.0\end{array}$ & $\begin{array}{l}-- \\
-- \\
-- \\
--\end{array}$ & $\begin{array}{l}-- \\
-- \\
-- \\
--\end{array}$ & $\begin{array}{l}-- \\
-- \\
-- \\
--\end{array}$ & $\begin{array}{l}-- \\
-- \\
-- \\
--\end{array}$ & $\begin{array}{r}18.7 \\
33.4 \\
12.8 \\
6.3 \\
17.9\end{array}$ & $\begin{array}{l}14.1 \\
14.7 \\
16.3 \\
16.9 \\
17.7\end{array}$ & $\begin{array}{l}252.96 \\
265.98 \\
268.70 \\
289.28 \\
313.28\end{array}$ \\
\hline $\begin{array}{l}P-31 \\
P-30 \\
P-29\end{array}$ & $\begin{array}{l}\text { Phosphate rock } \\
\text { Phosphate rock } \\
\text { Phosphate rock, argillaceous }\end{array}$ & $\begin{array}{l}\text { 2684-WOM } \\
2683-\text { WOM } \\
2667-\text { WOM }\end{array}$ & $\begin{array}{l}0.2 \\
0.3 \\
0.7\end{array}$ & $\begin{array}{l}29.1 \\
33.5 \\
25.8\end{array}$ & $\begin{array}{l}-- \\
\because-\end{array}$ & $\begin{array}{l}-- \\
--\end{array}$ & $\begin{array}{l}-- \\
--\end{array}$ & $\begin{array}{l}-- \\
--\end{array}$ & $\begin{array}{r}16.5 \\
6.9 \\
22.8\end{array}$ & $\begin{array}{l}17.9 \\
18.2 \\
18.9\end{array}$ & $\begin{array}{l}319.10 \\
329.15 \\
347.21\end{array}$ \\
\hline \multicolumn{12}{|c|}{ Pit $C$ north } \\
\hline \multicolumn{12}{|c|}{ Beds P-32 through P-29 were resampled in Pit C north; they are equivalent to beds of the same number in Pit E. } \\
\hline $\begin{array}{l}P-32 \\
P-31 \\
P-30 \\
P-29 \\
P-28\end{array}$ & $\begin{array}{l}\text { Phosphate rock } \\
\text { Phosphate rock } \\
\text { Phosphate rock } \\
\text { Phosphate rock } \\
\text { Phosphate rock }\end{array}$ & $\begin{array}{l}2682-\text { WOM } \\
2681-\text { WOM } \\
2680-W O M \\
2679-W O M \\
2678-\text { WOM }\end{array}$ & $\begin{array}{l}(0.7) \\
(0.5) \\
(0.5) \\
(0.7) \\
1.2\end{array}$ & $\begin{array}{l}34.4 \\
29.0 \\
33.9 \\
28.1 \\
32.9\end{array}$ & $\begin{array}{l}0.87 \\
1.9 \\
1.2 \\
3.3 \\
1.6\end{array}$ & $\begin{array}{l}0.59 \\
0.85 \\
0.48 \\
1.2 \\
0.71\end{array}$ & $\begin{array}{l}-- \\
-- \\
-- \\
--\end{array}$ & $\begin{array}{l}3.36 \\
4.60 \\
4.32 \\
5.78 \\
4.26\end{array}$ & $\begin{array}{r}7.4 \\
18.2 \\
8.2 \\
18.6 \\
10.0\end{array}$ & $\begin{array}{c}-- \\
-- \\
-- \\
20.1\end{array}$ & $\begin{array}{l}=- \\
=- \\
\overline{--} \\
386.69\end{array}$ \\
\hline $\begin{array}{l}P-27 \\
P-26 \\
P-25 \\
P-24 \\
P-23\end{array}$ & $\begin{array}{l}\text { Phosphate rock } \\
\text { Phosphate rock, argillaceous } \\
\text { Mudstone, phosphatic } \\
\text { Phosphate rock, argillaceous } \\
\text { Phosphate rock, argillaceous }\end{array}$ & $\begin{array}{l}2677-\text { WOM } \\
2676-\text { WOM } \\
2675-\text { WOM } \\
2674-\text { WOM } \\
2673-\text { WOM }\end{array}$ & $\begin{array}{l}0.5 \\
0.6 \\
0.4 \\
0.6 \\
0.7\end{array}$ & $\begin{array}{r}28.5 \\
25.2 \\
8.3 \\
23.7 \\
11.9\end{array}$ & $\begin{array}{l}-- \\
-- \\
-- \\
--\end{array}$ & $\begin{array}{l}-- \\
-- \\
-- \\
--\end{array}$ & $\begin{array}{l}-- \\
-- \\
--\end{array}$ & $\begin{array}{l}-- \\
-- \\
-- \\
--\end{array}$ & $\begin{array}{l}19.8 \\
28.9 \\
68.9 \\
31.7 \\
41.0\end{array}$ & $\begin{array}{l}20.6 \\
21.2 \\
21.6 \\
22.2 \\
22.9\end{array}$ & $\begin{array}{l}400.94 \\
416.06 \\
419.38 \\
433.60 \\
446.83\end{array}$ \\
\hline $\begin{array}{l}P-22 \\
P-21 \\
P-20\end{array}$ & $\begin{array}{l}\text { Phosphate rock, argillaceous } \\
\text { Phosphate rock, argillaceous } \\
\text { Mudstone, phosphatic }\end{array}$ & $\begin{array}{l}2672-\text { WOM } \\
2671-\text { WOM } \\
2670-\text { WOM }\end{array}$ & $\begin{array}{l}1.2 \\
1.1 \\
1.2\end{array}$ & $\begin{array}{r}27.4 \\
24.3 \\
8.8\end{array}$ & $\begin{array}{l}-- \\
--\end{array}$ & $\begin{array}{l}-- \\
-- \\
-\end{array}$ & $\begin{array}{l}-- \\
--\end{array}$ & $\begin{array}{l}-- \\
--\end{array}$ & $\begin{array}{l}23.2 \\
30.4 \\
60.2\end{array}$ & $\begin{array}{l}24.1 \\
25.2 \\
26.4\end{array}$ & $\begin{array}{l}479.71 \\
506.44 \\
517.00\end{array}$ \\
\hline
\end{tabular}




\begin{tabular}{|c|c|c|c|c|c|c|c|c|c|c|c|}
\hline \multirow[b]{2}{*}{$\begin{array}{l}\text { Bed } \\
\text { no. }\end{array}$} & \multirow[b]{2}{*}{ Rock description } & \multirow[b]{2}{*}{$\begin{array}{c}\text { Sample } \\
\text { no. }\end{array}$} & \multirow[b]{2}{*}{$\begin{array}{c}\text { Thickness } \\
\text { (feet) }\end{array}$} & \multicolumn{6}{|c|}{ Chemical analyses (percent) } & \multirow{2}{*}{$\begin{array}{l}\text { Cumulative } \\
\text { thickness } \\
\text { (feet) }\end{array}$} & \multirow{2}{*}{$\begin{array}{l}\text { Thickness } x \\
\text { percent } \mathrm{P}_{2} \mathrm{O}_{5} \\
\text { (cumulative) }\end{array}$} \\
\hline & & & & $\mathrm{P}_{2} \mathrm{O}_{5}$ & $\mathrm{Al}_{2} \mathrm{O}_{3}$ & $\mathrm{Fe}_{2} \mathrm{O}_{3}$ & $F$ & $\begin{array}{l}\text { Loss on } \\
\text { ignition }\end{array}$ & $\begin{array}{c}\text { Acid } \\
\text { insoluble }\end{array}$ & & \\
\hline $\begin{array}{l}P-19 \\
P-18 \\
P-17 \\
P-16 \\
--\end{array}$ & $\begin{array}{l}\text { Mudstone, phosphatic } \\
\text { Phosphate rock, argillaceous } \\
\text { Phosphate rock } \\
\text { Phosphate rock, argillaceous } \\
\text { Chert concretion }\end{array}$ & $\begin{array}{l}\text { 2669-WOM } \\
2668-\text { WOM } \\
2666-\text { WOM } \\
2665-\text { WOM } \\
2664-\text { WOM }\end{array}$ & $\begin{array}{l}1.9 \\
0.4 \\
1.4 \\
1.4 \\
(0.6)\end{array}$ & $\begin{array}{r}14.5 \\
23.8 \\
34.0 \\
21.7 \\
7.2\end{array}$ & $\begin{array}{l}-- \\
3.3 \\
0.74 \\
2.8 \\
0.92\end{array}$ & $\begin{array}{l}1.3 \\
0.43 \\
1.1 \\
1.9\end{array}$ & $\begin{array}{l}-- \\
-- \\
-- \\
--\end{array}$ & $\begin{array}{l}-\overline{-} \\
5.48 \\
5.28 \\
5.72 \\
2.70\end{array}$ & $\begin{array}{r}52.5 \\
29.5 \\
7.0 \\
36.0 \\
75.2\end{array}$ & $\begin{array}{l}28.3 \\
28.7 \\
30.1 \\
31.5 \\
75.2\end{array}$ & $\begin{array}{l}544.55 \\
554.07 \\
601.67 \\
632.05 \\
--\end{array}$ \\
\hline $\begin{array}{l}P-15 \\
P-14 \\
P-13 \\
P-12 \\
P-11\end{array}$ & $\begin{array}{l}\text { Phosphate rock, argillaceous } \\
\text { Phosphate rock } \\
\text { Phosphate rock } \\
\text { Phosphate rock, argillaceous } \\
\text { Phosphate rock }\end{array}$ & $\begin{array}{l}\text { 2663-WOM } \\
2662-\text { WOM } \\
2661-\text { WOM } \\
2660-\text { WOM } \\
2659-\text { WOM }\end{array}$ & $\begin{array}{l}1.3 \\
0.8 \\
1.5 \\
0.8 \\
0.7\end{array}$ & $\begin{array}{l}26.3 \\
31.7 \\
29.4 \\
23.2 \\
31.6\end{array}$ & $\begin{array}{l}3.0 \\
1.3 \\
2.3 \\
6.0 \\
2.2\end{array}$ & $\begin{array}{l}1.1 \\
0.59 \\
0.84 \\
1.7 \\
0.77\end{array}$ & $\begin{array}{l}-- \\
-- \\
--\end{array}$ & $\begin{array}{l}5.46 \\
4.56 \\
5.84 \\
5.30 \\
4.36\end{array}$ & $\begin{array}{l}24.9 \\
12.1 \\
16.3 \\
31.6 \\
13.4\end{array}$ & $\begin{array}{l}32.8 \\
33.6 \\
35.1 \\
35.9 \\
36.6\end{array}$ & $\begin{array}{l}666.24 \\
691.60 \\
735.70 \\
754.26 \\
776.38\end{array}$ \\
\hline $\begin{array}{l}P-10 \\
P-9 \\
P-8 \\
P-7 \\
P-6\end{array}$ & $\begin{array}{l}\text { Phosphate rock, argillaceous } \\
\text { Phosphate rock, argillaceous } \\
\text { Phosphate rock, argillaceous } \\
\text { Phosphate rock } \\
\text { Phosphate rock }\end{array}$ & $\begin{array}{l}2658-\text { WOM } \\
2657-\text { WOM } \\
2656-\text { WOM } \\
2655-\text { WOM } \\
2654-\text { WOM }\end{array}$ & $\begin{array}{l}2.5 \\
0.4 \\
0.7 \\
2.4 \\
3.7\end{array}$ & $\begin{array}{l}24.4 \\
19.2 \\
25.3 \\
35.1 \\
31.9\end{array}$ & $\begin{array}{l}3.6 \\
6.1 \\
4.5 \\
0.61 \\
0.89\end{array}$ & $\begin{array}{l}1.1 \\
2.1 \\
2.0 \\
0.53 \\
0.55\end{array}$ & $\begin{array}{l}-- \\
-- \\
-- \\
--\end{array}$ & $\begin{array}{l}4.52 \\
4.50 \\
3.88 \\
4.40 \\
4.84\end{array}$ & $\begin{array}{r}29.2 \\
38.8 \\
26.0 \\
3.5 \\
10.2\end{array}$ & $\begin{array}{l}39.1 \\
39.5 \\
40.2 \\
42.6 \\
46.3\end{array}$ & $\begin{array}{r}837.38 \\
845.06 \\
862.77 \\
947.01 \\
1,065.04\end{array}$ \\
\hline $\begin{array}{l}P=5 \\
P=4 \\
P=3 \\
P=2 \\
P=1\end{array}$ & $\begin{array}{l}\text { Phosphate rock, argillaceous } \\
\text { Mudstone } \\
\text { Phosphate rock } \\
\text { Mudstone and chert } \\
\text { Mudstone, phosphatic }\end{array}$ & $\begin{array}{l}2653-\text { WOM } \\
2652-\text { WOM } \\
2651-\text { WOM } \\
2650-\text { WOM } \\
2280-\text { WOM }\end{array}$ & $\begin{array}{l}0.8 \\
2.5 \\
0.4 \\
1.7 \\
0.9\end{array}$ & $\begin{array}{r}18.3 \\
2.9 \\
30.4 \\
2.6 \\
43.9\end{array}$ & $\begin{array}{l}-- \\
-- \\
-- \\
-- \\
--\end{array}$ & $\begin{array}{l}-- \\
-- \\
-- \\
--\end{array}$ & $\begin{array}{l}-- \\
=- \\
-- \\
-\end{array}$ & $\begin{array}{l}-- \\
-- \\
-- \\
--\end{array}$ & $\begin{array}{l}38.2 \\
78.2 \\
15.9 \\
83.4 \\
50.5\end{array}$ & $\begin{array}{l}47.1 \\
49.6 \\
50.0 \\
51.7 \\
52.6\end{array}$ & $\begin{array}{l}1,079.68 \\
1,086.93 \\
1,099.09 \\
1,103.51 \\
1,116.02\end{array}$ \\
\hline
\end{tabular}


WOODALL CREEK, IDAHO. LOT NO. 1262.

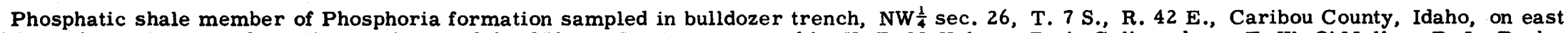

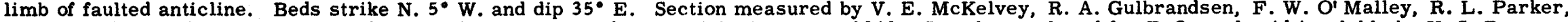

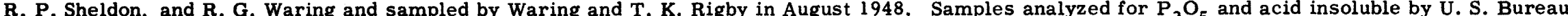

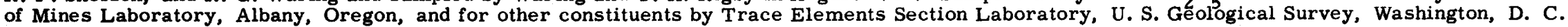

\begin{tabular}{|c|c|c|c|c|c|c|c|c|c|c|}
\hline \multirow{2}{*}{$\begin{array}{l}\text { Bed } \\
\text { no. }\end{array}$} & \multirow[b]{2}{*}{ Rock description } & \multirow{2}{*}{$\begin{array}{c}\text { Sample } \\
\text { no. }\end{array}$} & \multirow{2}{*}{$\begin{array}{c}\text { Thickness } \\
\text { (feet) }\end{array}$} & \multicolumn{5}{|c|}{ Chemical analyses (percent) } & \multirow{2}{*}{$\begin{array}{c}\text { Cumulative } \\
\text { thickness } \\
\text { (feet) }\end{array}$} & \multirow{2}{*}{$\begin{array}{l}\text { Thickness } \mathbf{x} \\
\text { percent } \mathrm{P}_{2} \mathrm{O}_{5} \\
\text { (cumulative) }\end{array}$} \\
\hline & & & & $\mathrm{P}_{2} \mathrm{O}_{5}$ & $\mathrm{Al}_{2} \mathrm{O}_{3}$ & $\mathrm{Fe}_{2} \mathrm{O}_{3}$ & $\begin{array}{l}\text { Loss on } \\
\text { ignition }\end{array}$ & $\begin{array}{c}\text { Acid } \\
\text { insoluble }\end{array}$ & & \\
\hline
\end{tabular}

Rex member of Phosphoria formation-basal beds only

\begin{tabular}{|c|c|c|c|c|c|c|c|c|c|c|}
\hline $\begin{array}{l}R-2 \\
R-1\end{array}$ & $\begin{array}{l}\text { Mudstone } \\
\text { Chert }\end{array}$ & $\begin{array}{l}1177-\text { WOM } \\
1176-\text { WOM }\end{array}$ & $\begin{array}{l}6.0 \\
2.0\end{array}$ & $\begin{array}{l}0.7 \\
0.7\end{array}$ & -- & $\begin{array}{l}-- \\
--\end{array}$ & -- & $\begin{array}{l}85.9 \\
91.7\end{array}$ & $\begin{array}{l}6.0 \\
8.0\end{array}$ & $\begin{array}{l}4.20 \\
5.60\end{array}$ \\
\hline \multicolumn{11}{|c|}{ Phosphatic shale member of Phosphoria formation } \\
\hline $\begin{array}{l}P-92 \\
P-91 \\
P-90 \\
P-89 \\
P-88\end{array}$ & $\begin{array}{l}\text { Mudstone } \\
\text { Mudstone; fos. col. no. } 48-J E S-205^{1} \\
\text { Mudstone } \\
\text { Mudstone; fos. col. no. } 48-J E S-204 \\
\text { Mudstone, phosphatic; fos. col. no. } \\
\quad 48-J E S-203\end{array}$ & $\begin{array}{l}1175-\text { WOM } \\
1174-\text { WOM } \\
1170-\text { WOM } \\
1169-\text { WOM } \\
1168-\text { WOM }\end{array}$ & $\begin{array}{l}1.1 \\
1.5 \\
0.6 \\
0.9 \\
1.5\end{array}$ & $\begin{array}{l}2.3 \\
0.6 \\
0.7 \\
3.1 \\
8.8\end{array}$ & $\begin{array}{l}-- \\
-- \\
-- \\
--\end{array}$ & $\begin{array}{l}-- \\
-- \\
-- \\
--\end{array}$ & $\begin{array}{l}-- \\
-- \\
-- \\
--\end{array}$ & $\begin{array}{l}79.5 \\
81.5 \\
79.8 \\
72.9 \\
63.2\end{array}$ & $\begin{array}{l}1.1 \\
2.6 \\
3.2 \\
4.1 \\
5.6\end{array}$ & $\begin{array}{r}2.53 \\
3.43 \\
3.85 \\
6.64 \\
19.84\end{array}$ \\
\hline $\begin{array}{l}P-87 \\
P-86 \\
P-85 \\
P-84\end{array}$ & $\begin{array}{l}\text { Phosphate rock, argillaceous; fos. } \\
\text { col. no. 48-JES-202 } \\
\text { Mudstone } \\
\text { Phosphate rock } \\
\text { Phosphate rock, argillaceous }\end{array}$ & $\begin{array}{l}1167-W O M \\
1166-W O M \\
1165-W O M \\
1164-W O M\end{array}$ & $\begin{array}{l}0.9 \\
0.8 \\
2.7 \\
0.5\end{array}$ & $\begin{array}{r}19.5 \\
5.0 \\
31.2 \\
24.2\end{array}$ & $\begin{array}{l}-- \\
-- \\
1.8 \\
5.3\end{array}$ & $\begin{array}{l}-- \\
-- \\
1.1 \\
1.9\end{array}$ & $\begin{array}{l}-- \\
-- \\
3.16 \\
4.08\end{array}$ & $\begin{array}{l}41.0 \\
68.5 \\
13.6 \\
25.9\end{array}$ & $\begin{array}{r}6.5 \\
7.3 \\
10.0 \\
10.5\end{array}$ & $\begin{array}{r}37.39 \\
41.39 \\
125.63 \\
137.73\end{array}$ \\
\hline
\end{tabular}

From 1164-WOM to top of section units are weathered, slumped, and poorly exposed making thicknesses measured of questionable value.

P-83 Phosphate rock, argillaceous

P-82 Phosphate rock and phosphatic mudstone

P-81 Phosphate rock

P-80 Phosphate rock, argillaceous

-79 Phosphate rock, argillaceous

P-78 Phosphate rock, argillaceous

P-77 Phosphate rock, argillaceous

P-76 Mudstone

P-75 Phosphate rock, argillaceous

P-74 Phosphate rock, argillaceous

P-73 Mudstone, phosphatic; fos. col. no. 48-JES-201

P-72 Mudstone

2000-WOM
$1999-$ WOM
$1998-$ WOM
$1997-$ WOM
$1996-$ WOM
$1995-$ WOM
$1994-$ WOM
$1993-$ WOM
$1992-$ WOM
$1991-$ WOM
$1990-$ WOM
$1989-$ WOM

0.4
0.5
0.6
1.2
3.7
1.9
1.8
1.0
1.2
0.4
3.3
1.9

\begin{tabular}{r|r|}
21.1 & 5.3 \\
19.1 & 5.7 \\
31.7 & 2.8 \\
23.1 & 4.4 \\
21.7 & 5.3 \\
22.8 & 5.3 \\
21.9 & 5.5 \\
7.1 & 9.5 \\
21.0 & 5.6 \\
27.1 & 1.8 \\
8.4 & -- \\
4.4 &.-
\end{tabular}

\begin{tabular}{r|}
5.34 \\
5.88 \\
6.10 \\
11.08 \\
13.38 \\
8.66 \\
6.30 \\
5.64 \\
6.02 \\
3.44 \\
--
\end{tabular}

34.1
39.0
10.9
24.9
25.8
27.9
33.2
73.0
35.3
24.3
68.2
79.0

10.9

11.4

12.0

13.2

16.9

18.8

20.6

21.6

22. 8

23. 2

26.5

28.4
146.17

155. 72

1 74. 74

202. 46

282.75

326.07

365.49

372.59

397.79

408.63

436.35

444.71 


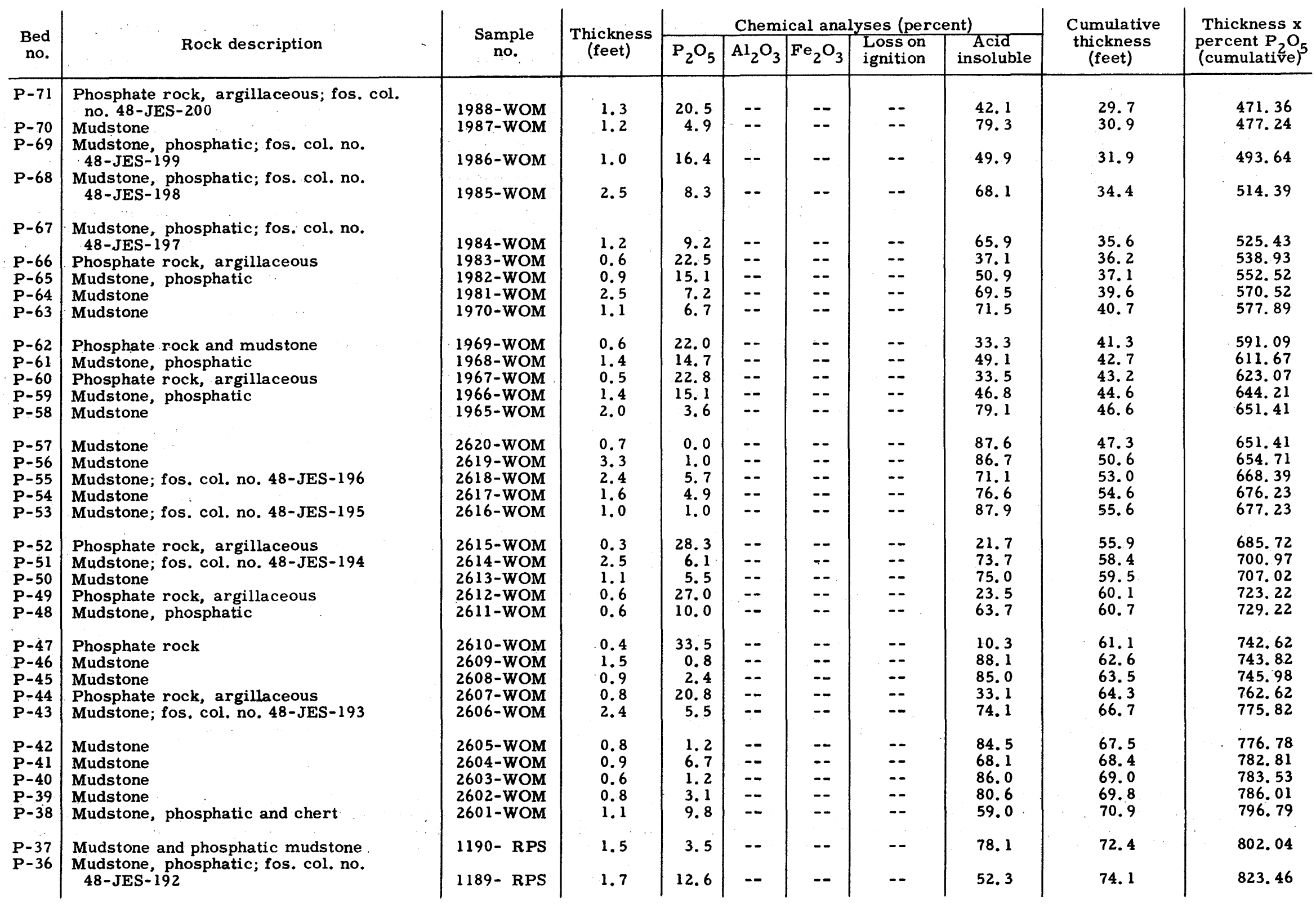




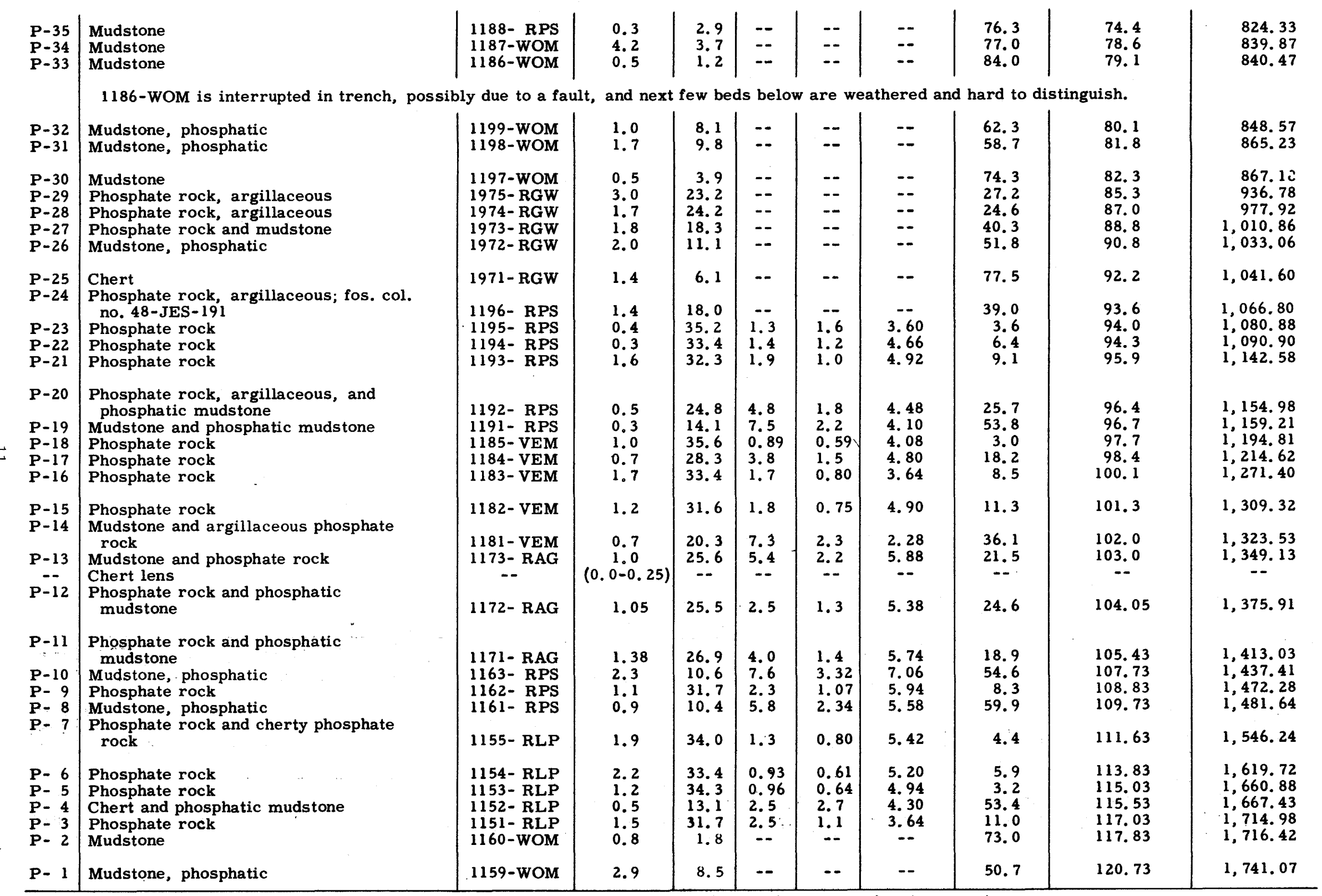

* Cumulative data incomplete due to missing information. Computations start from zero after interruption.

** Note incompleteness of cumulative data. 


\begin{tabular}{|c|c|c|c|c|c|c|c|c|c|c|}
\hline \multirow[b]{2}{*}{$\begin{array}{c}\text { Bed } \\
\text { no. }\end{array}$} & \multirow[b]{2}{*}{ Rock description } & \multirow[b]{2}{*}{$\begin{array}{c}\text { Sample } \\
\text { no. }\end{array}$} & \multirow{2}{*}{$\begin{array}{c}\text { Thickness } \\
\text { (feet) }\end{array}$} & \multicolumn{5}{|c|}{ Chemical analyses (percent) } & \multirow{2}{*}{$\begin{array}{l}\text { Cumulative } \\
\text { thickness } \\
\text { (feet) }\end{array}$} & \multirow{2}{*}{$\begin{array}{l}\text { Thickness } \mathrm{x} \\
\text { percent } \mathrm{P}_{2} \mathrm{O}_{5} \\
\text { (cumulative) }\end{array}$} \\
\hline & & & & $\mathrm{P}_{2} \mathrm{O}_{5}$ & $\mathrm{Al}_{2} \mathrm{O}_{3}$ & $\mathrm{Fe}_{2} \mathrm{O}_{3}$ & $\begin{array}{l}\text { Loss on } \\
\text { ignition }\end{array}$ & $\begin{array}{c}\text { Acid } \\
\text { insoluble }\end{array}$ & & \\
\hline \multicolumn{11}{|c|}{ Wells formation - top beds only } \\
\hline \multirow[t]{2}{*}{$\begin{array}{l}\mathrm{Cw}-1 \\
\mathrm{Cw}-2\end{array}$} & $\begin{array}{l}\text { Chert } \\
\text { Mudstone }\end{array}$ & $\begin{array}{l}\text { 1158-WOM } \\
1157-W O M\end{array}$ & $\begin{array}{l}3.5 \\
3.6\end{array}$ & $\begin{array}{l}4.6 \\
3.9\end{array}$ & $=-$ & -- & -- & $\begin{array}{l}77.3 \\
57.9\end{array}$ & $\begin{array}{l}3.5 \\
7.1\end{array}$ & $\begin{array}{l}16.10 \\
30.14\end{array}$ \\
\hline & Unit $1157-$ WOM contains gouge and & possibly fo & med by faul & ing. & & & & & & \\
\hline $\mathrm{CW}-3$ & Limestone; fos. col. no. 48-JES-190 & 1156-WOM & 0.7 & 0.39 & -. & -- & -- & 4.6 & 7.8 & 30.41 \\
\hline
\end{tabular}


BLACKFOOT NARROWS, IDAHO. LOT NO. 1261.

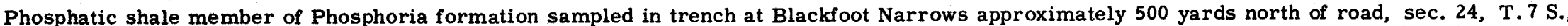

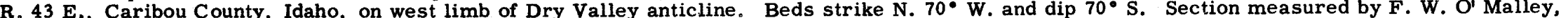

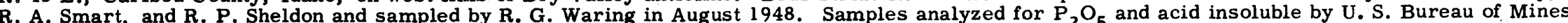
Laboratory, Albany, Oregon, and for other constituents by Trace Elements Section Laboratory, U. S. Geological Survey, Washington, D. C.

\begin{tabular}{|c|c|c|c|c|c|c|c|c|c|c|}
\hline \multirow{2}{*}{$\begin{array}{c}\text { Bed } \\
\text { no. }\end{array}$} & \multirow[b]{2}{*}{ Rock description } & \multirow{2}{*}{$\begin{array}{c}\text { Sample } \\
\text { no. }\end{array}$} & \multirow{2}{*}{$\begin{array}{c}\text { Thickness } \\
\text { (feet) }\end{array}$} & \multicolumn{5}{|c|}{ Chemical analyses (percent) } & \multirow{2}{*}{$\begin{array}{c}\text { Cumulative } \\
\text { thickness } \\
\text { (feet) }\end{array}$} & \multirow{2}{*}{$\begin{array}{l}\text { Thickness } \mathrm{x} \\
\text { percent } \mathrm{P}_{2} \mathrm{O}_{5} \\
\text { (cumulative) }\end{array}$} \\
\hline & & & & $\mathrm{P}_{2} \mathrm{O}_{5}$ & $\mathrm{Al}_{2} \mathrm{O}_{3}$ & $\mathrm{Fe}_{2} \mathrm{O}_{3}$ & $\begin{array}{l}\text { Loss on } \\
\text { ignition }\end{array}$ & $\begin{array}{c}\text { Acid } \\
\text { insoluble }\end{array}$ & & \\
\hline
\end{tabular}

\begin{tabular}{ll|l}
\hline$R-8$ & Chert, argillaceous \\
$\mathbf{R}-$ & 7 & Mudstone, cherty \\
$\mathbf{R}-$ & 6 & Mudstone \\
$\mathbf{R}-$ & 5 & Chert, argillaceous \\
$\mathbf{R}-$ & 4 & Mudstone \\
$\mathbf{R}-$ & 3 & Chert \\
$\mathbf{R}-$ & 2 & Mudstone \\
$\mathbf{R}-$ & $\mathbf{1}$ & Chert \\
\hline
\end{tabular}

Rex member of Phosphoria formation_basal beds only

\begin{tabular}{|c|l|l|l|l|l|l|l|l}
\hline $1940-$ WOM & 3.0 & 1.3 & -- & -- & -- & 89.2 & 3.0 & 3.90 \\
$1939-$ WOM & 1.0 & 0.5 & -- & -- & -- & 88.5 & 4.0 & 4.40 \\
$1938-$ WOM & 0.9 & 0.5 & -- & -- & -- & 77.8 & 4.9 & 4.85 \\
$1937-$ WOM & 0.5 & 0.6 & -- & -- & -- & 89.7 & 5.4 & 5.15 \\
$1936-$ WOM & 0.8 & 0.4 & -- & -- & -- & 82.5 & 6.2 & 5.47 \\
& & & & & & & \\
$1935-$ WOM & 2.3 & 2.8 & -- & -- & -- & 81.9 & 8.5 & 11.91 \\
$1934-$ WOM & 0.7 & 0.3 & -- & -- & -- & 82.7 & 9.2 & 12.12 \\
$1933-$ WOM & 2.4 & 6.5 & -- & -- & -- & 72.7 & 11.6 & 27.72 \\
\hline
\end{tabular}

Phosphatic shale member of Phosphoria formation

\begin{tabular}{l|l}
\hline P-114 & Mudstone, calcareous, phosphatic \\
P-113 & Mudstone \\
P-112 & Mudstone \\
P-111 & Mudstone; fos. col. no. 48-JES-147 \\
P-110 & Phosphate rock, argillaceous \\
& \\
P-109 & Mudstone; fos. col. no. 48-JES-146 \\
P-108 & Mudstone; fos. col. no. 48-JES-145 \\
P-107 & Phosphate rock \\
P-106 & Phosphate rock, argillaceous \\
P-105 & Mudstone, phosphatic \\
P-104 & Phosphate rock \\
P-103 & Mudstone \\
P-102 & Phosphate rock \\
P-101 & Phosphate rock \\
P-100 & Phosphate rock \\
P- 99 & Phosphate rock, argillaceous; fos. \\
P- 98 & col. no. 48-JES-144 \\
P-97 & Phosphate rock \\
P- 96 & Phosphate rock \\
&
\end{tabular}

\begin{tabular}{|l}
$1932-$ WOM \\
$1931-$ WOM \\
$1950-$ WOM \\
$1949-$ WOM \\
$1948-$ WOM \\
$1947-$ WOM \\
$1946-$ WOM \\
$1945-$ WOM \\
$1944-$ WOM \\
$1943-$ WOM \\
$1942-$ WOM \\
$1941-$ WOM \\
$1930-$ WOM \\
$1929-$ WOM \\
$1960-$ WOM \\
$1959-$ WOM \\
$1958-$ WOM \\
$1957-$ WOM \\
$1956-$ WOM
\end{tabular}

\begin{tabular}{l|r|l|l|}
0.3 & 9.8 & -- & -- \\
1.0 & 2.5 & -- & -- \\
1.5 & 1.3 & -- & -- \\
3.0 & 2.6 & -- & -- \\
0.8 & 28.1 & -- & -- \\
2.5 & 2.7 & -- & -- \\
3.0 & 0.7 & -- & -- \\
0.6 & 34.5 & 1.2 & 0.44 \\
0.3 & 18.2 & 6.4 & 2.4 \\
0.6 & 12.8 & 6.9 & 3.0 \\
0.7 & 32.1 & 1.4 & 1.0 \\
0.3 & 6.4 & 9.4 & 2.9 \\
0.9 & 30.2 & 2.5 & 1.1 \\
0.6 & 31.7 & 1.4 & 0.85 \\
0.5 & 28.5 & 3.1 & 1.6 \\
& & & \\
2.9 & 26.8 & 3.7 & 0.56 \\
0.4 & 33.4 & 1.6 & 0.67 \\
2.4 & 32.5 & 2.0 & 0.71 \\
4.8 & 27.9 & 3.0 & 2.3 \\
& & &
\end{tabular}

--
--
--
--
--
--
2.16
5.68
5.30
2.88
6.44
4.42
3.18
4.38

6.28
4.60
6.06
9.42

48.3
74.2
74.5
70.9
21.8
71.1
77.5
10.1
41.3
54.3
13.3
65.3
16.1
14.7
19.9

22.7
7.8
8.3
14.1

0.3
1.3
2.8
5.8
6.6
9.1
12.1
12.7
13.0
13.6
14.3
14.6
15.5
16.1
16.6

19.5
19.9
22.3
27.1

2.94 5.44 7.39 15.19

${ }^{1}$ Fossil collection made by J. E. Smedley, Paleontology and Stratigraphy Branch, U. S. Geological Survey.

44.42

46.52

67.22

72.68

80.36

102.83 104.75

131.93

150.95

165.20

242.92

256.28

334.28 468.20 


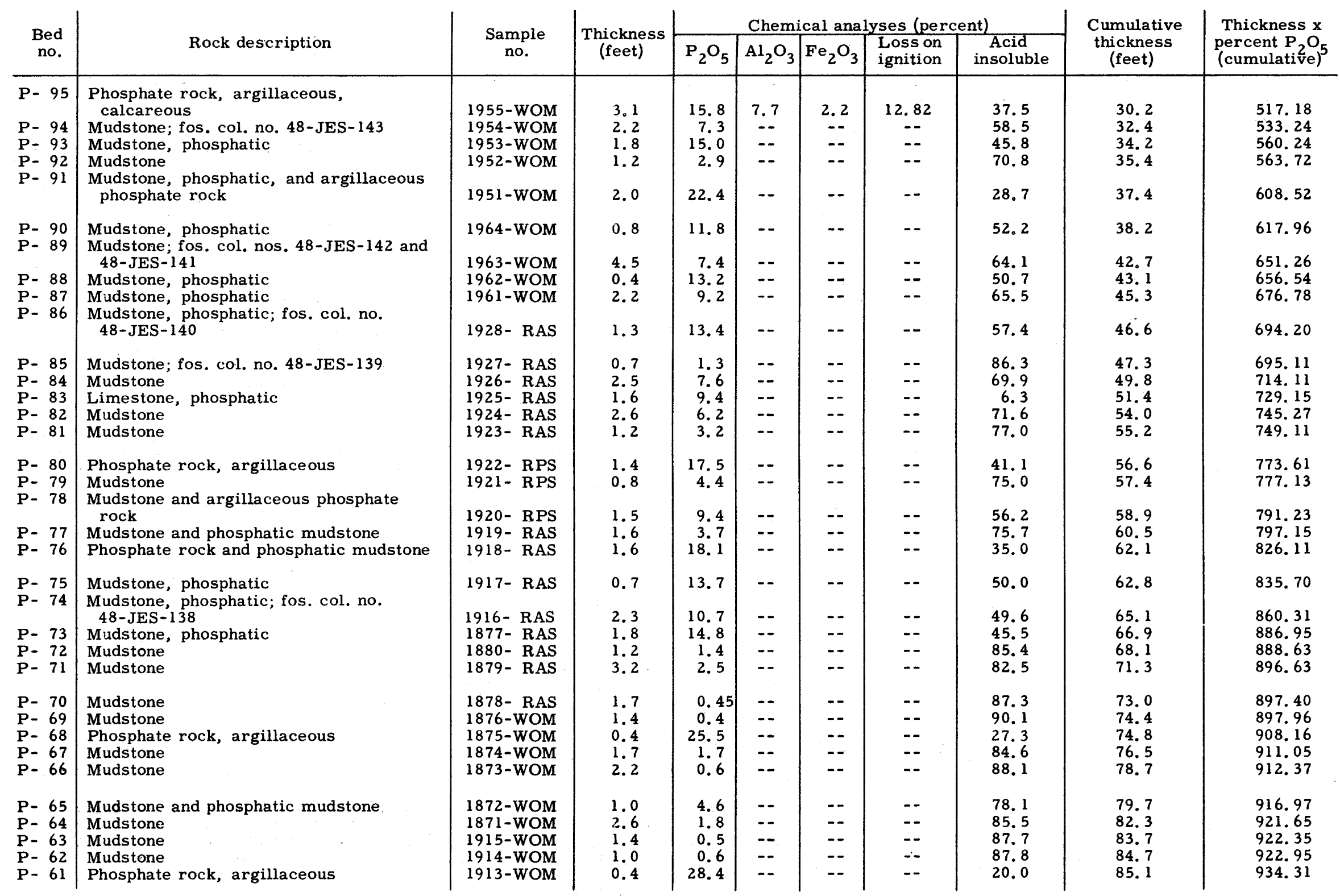




\begin{tabular}{|c|c|}
\hline$P-60$ & Mudstone \\
\hline$P-59$ & Phosphate rock, argillaceous \\
\hline $\begin{array}{l}P-58 \\
P-57\end{array}$ & $\begin{array}{l}\text { Mudstone } \\
\text { Phosnhate rock a roillaceous }\end{array}$ \\
\hline P- 56 & Mudstone \\
\hline$P-55$ & Phosphate rock \\
\hline P- 54 & Mudstone \\
\hline$P-53$ & Phosphate rock \\
\hline $\begin{array}{l}P-52 \\
P-51\end{array}$ & $\begin{array}{l}\text { Muds tone } \\
\text { Phosphate rock, argillaceous }\end{array}$ \\
\hline & Mudatono \\
\hline $\begin{array}{l}P-50 \\
P-49\end{array}$ & $\begin{array}{l}\text { Muastone } \\
\text { Mudstone }\end{array}$ \\
\hline$P-48$ & Mudstone, phosphatic \\
\hline P. 47 & Mudstone piluspialic \\
\hline$P-46$ & Mudstone \\
\hline P- 45 & Mudstone, phosphatic \\
\hline P- 44 & Mudstone, phosphatic \\
\hline P- 43 & Mudstone \\
\hline$P-42$ & Mudstone, phosphatic \\
\hline$P-41$ & Mudstone, phosphatic \\
\hline$P-40$ & Mudstone and chert \\
\hline P- 39 & Mudstone, phosphatic \\
\hline P- 38 & $\begin{array}{l}\text { Mudstone; fos. col nos. } 438 \text {-JES- } 137 \text { and } \\
\text { 48-JES-131 }\end{array}$ \\
\hline $\begin{array}{l}P-37 \\
P-36\end{array}$ & $\begin{array}{l}\text { Mudstone; fos. col. no. } 48-J E S-130 \\
\text { Mudstone }\end{array}$ \\
\hline P- 35 & Mudstone; fos. col. no. 48-JES-132 \\
\hline P- 34 & Mudstone \\
\hline P- 33 & Mudstone; fos. col. no. 48-JES-129 \\
\hline P- & Mudstone \\
\hline P- 31 & Mudstone; fos. col. no. 48-JES-133 \\
\hline & $\begin{array}{l}\text { Limestone, argillaceous, phosphatic; } \\
\text { fos. col. no. } 48-\mathrm{JES}-134\end{array}$ \\
\hline$P-29$ & Mudstone, phosphatic \\
\hline $\begin{array}{l}P-28 \\
P=27\end{array}$ & $\begin{array}{l}\text { Mudstone, phosphatic } \\
\text { Mudstone }\end{array}$ \\
\hline$P-26$ & Mudstone, phosphatic \\
\hline & $\begin{array}{l}\text { Mudstone, phosphatic; fos. col. no. } \\
\text { 48-JES-135 }\end{array}$ \\
\hline P- 24 & Phosphate rock, argillaceous \\
\hline$P-23$ & Phosphate rock \\
\hline & Mudstone, phosphatic \\
\hline P- 21 & Phosphate rock \\
\hline P- & $\begin{array}{l}\text { Mudstone, phosphatic, and argillaceous } \\
\text { phosphate rock }\end{array}$ \\
\hline
\end{tabular}

\begin{tabular}{|c|c|c|c|c|c|c|c|c|}
\hline $\begin{array}{l}\text { 1912-WOM } \\
1911-W O M \\
1910-W O M \\
1909-W O M \\
1908-W O M\end{array}$ & $\begin{array}{l}0.5 \\
0.4 \\
2.5 \\
0.4 \\
1.6\end{array}$ & $\begin{array}{r}5.5 \\
22.1 \\
1.2 \\
27.3 \\
1.6\end{array}$ & $\begin{array}{l}-- \\
-- \\
-- \\
--\end{array}$ & $\begin{array}{l}-- \\
-- \\
-- \\
--\end{array}$ & $\begin{array}{l}-- \\
-- \\
-- \\
--\end{array}$ & $\begin{array}{l}70.6 \\
36.5 \\
85.7 \\
21.4 \\
84.3\end{array}$ & $\begin{array}{l}85.6 \\
86.0 \\
88.5 \\
88.9 \\
90.5\end{array}$ & $\begin{array}{l}937.06 \\
945.90 \\
948.90 \\
959.82 \\
962.38\end{array}$ \\
\hline $\begin{array}{l}1907-W O M \\
1906-W O M \\
1905-W O M \\
1904-W O M \\
1903-W O M\end{array}$ & $\begin{array}{l}0.3 \\
0.8 \\
0.4 \\
2.0 \\
0.3\end{array}$ & $\begin{array}{r}30.3 \\
1.6 \\
30.6 \\
5.9 \\
21.4\end{array}$ & $\begin{array}{l}-- \\
-- \\
-- \\
-- \\
--\end{array}$ & $\begin{array}{l}-- \\
-- \\
-- \\
--\end{array}$ & $\begin{array}{l}-- \\
-- \\
-- \\
--\end{array}$ & $\begin{array}{l}15.5 \\
82.4 \\
15.3 \\
74.3 \\
34.3\end{array}$ & $\begin{array}{l}90.8 \\
91.6 \\
92.0 \\
94.0 \\
94.3\end{array}$ & $\begin{array}{r}971.47 \\
972.75 \\
984.99 \\
996.79 \\
1,003.21\end{array}$ \\
\hline $\begin{array}{l}1902-W O M \\
1901-W O M \\
1900-W O M \\
1899-W O M \\
1898-W O M\end{array}$ & $\begin{array}{l}0.8 \\
0.8 \\
1.2 \\
0.6 \\
0.8\end{array}$ & $\begin{array}{r}7.7 \\
0.6 \\
10.7 \\
0.6 \\
4.4\end{array}$ & $\begin{array}{l}-- \\
-- \\
-- \\
-- \\
--\end{array}$ & $\begin{array}{l}-- \\
-- \\
-- \\
--\end{array}$ & $\begin{array}{l}-- \\
=- \\
=- \\
--\end{array}$ & $\begin{array}{l}63.1 \\
87.4 \\
55.2 \\
88.5 \\
73.8\end{array}$ & $\begin{array}{l}95.1 \\
95.9 \\
97.1 \\
97.7 \\
98.5\end{array}$ & $\begin{array}{l}1,009.37 \\
1,009.85 \\
1,022.69 \\
1,023.05 \\
1,026.57\end{array}$ \\
\hline $\begin{array}{l}1897-W O M \\
1896-W O M \\
1895-W O M \\
1894-W O M \\
1893-W O M\end{array}$ & $\begin{array}{l}3.3 \\
2.5 \\
0.4 \\
1.2 \\
3.0\end{array}$ & $\begin{array}{r}13.1 \\
10.3 \\
2.6 \\
10.1 \\
8.3\end{array}$ & $\begin{array}{l}-- \\
-- \\
-- \\
-- \\
--\end{array}$ & $\begin{array}{l}-- \\
-- \\
--\end{array}$ & $\begin{array}{l}-- \\
-- \\
-- \\
-- \\
--\end{array}$ & $\begin{array}{l}35.1 \\
53.6 \\
78.6 \\
45.9 \\
52.6\end{array}$ & $\begin{array}{l}101.8 \\
104.3 \\
104.7 \\
105.9 \\
108.9\end{array}$ & $\begin{array}{l}1,069.80 \\
1,095.55 \\
1,096.59 \\
1,108.71 \\
1,133.61\end{array}$ \\
\hline $\begin{array}{l}\text { 1892-WOM } \\
1891-W O M\end{array}$ & $\begin{array}{l}0.9 \\
2.0\end{array}$ & $\begin{array}{r}6.6 \\
11.9\end{array}$ & $\begin{array}{l}-- \\
--\end{array}$ & -- & -- & $\begin{array}{l}59.3 \\
49.4\end{array}$ & $\begin{array}{l}109.8 \\
111.8\end{array}$ & $\begin{array}{l}1,139.55 \\
1,163.35\end{array}$ \\
\hline $\begin{array}{l}\text { 1890-WOM } \\
1889-\text { WOM } \\
1888-\text { WOM }\end{array}$ & $\begin{array}{l}2.0 \\
1.1 \\
2.0\end{array}$ & $\begin{array}{l}6.8 \\
1.7 \\
7.1\end{array}$ & $\begin{array}{l}-- \\
--\end{array}$ & $\begin{array}{l}-- \\
--\end{array}$ & $\begin{array}{l}-- \\
-- \\
--\end{array}$ & $\begin{array}{l}64.5 \\
81.6 \\
65.0\end{array}$ & $\begin{array}{l}113.8 \\
114.9 \\
116.9\end{array}$ & $\begin{array}{l}1,176.95 \\
1,178.82 \\
1,193.02\end{array}$ \\
\hline $\begin{array}{l}1887-\text { WOM } \\
1886-\text { WOM } \\
1885-\text { WOM } \\
1884-W O M\end{array}$ & $\begin{array}{l}0.6 \\
4.2 \\
3.7 \\
0.8\end{array}$ & $\begin{array}{l}3.6 \\
1.9 \\
5.5 \\
0.5\end{array}$ & $\begin{array}{l}-- \\
-- \\
-- \\
--\end{array}$ & $\begin{array}{l}-- \\
-- \\
-- \\
--\end{array}$ & $\begin{array}{l}-- \\
-- \\
-- \\
--\end{array}$ & $\begin{array}{l}79.1 \\
79.0 \\
70.7 \\
87.9\end{array}$ & $\begin{array}{l}117.5 \\
121.7 \\
125.4 \\
126.2\end{array}$ & $\begin{array}{l}1,195.18 \\
1,203.16 \\
1,223.51 \\
1,223.91\end{array}$ \\
\hline 1883-WOM & 0.8 & 1.2 & -- & - & -- & 84.8 & 127.0 & $1,224.87$ \\
\hline $\begin{array}{l}1882-W O M \\
1881-W O M \\
1870-W O M \\
1869-W O M\end{array}$ & $\begin{array}{l}3.7 \\
0.5 \\
3.0 \\
0.9\end{array}$ & $\begin{array}{r}9.6 \\
12.3 \\
16.1 \\
4.0\end{array}$ & $\begin{array}{l}-- \\
-- \\
-- \\
--\end{array}$ & $\begin{array}{l}-- \\
-- \\
--\end{array}$ & $\begin{array}{l}-- \\
-- \\
-- \\
--\end{array}$ & $\begin{array}{l}25.8 \\
54.4 \\
41.0 \\
78.0\end{array}$ & $\begin{array}{l}130.7 \\
131.2 \\
134.2 \\
135.1\end{array}$ & $\begin{array}{l}1,260.39 \\
1,266.54 \\
1,314.84 \\
1,318.44\end{array}$ \\
\hline 1868-WOM & 1.7 & 8.0 & -- & - & -- & 64.2 & 136.8 & $1,332.04$ \\
\hline $\begin{array}{l}1867-\text { WOM } \\
1866-\text { WOM } \\
1865-\text { WOM } \\
1864-\text { WOM }\end{array}$ & $\begin{array}{l}2.3 \\
2.0 \\
1.2 \\
2.6\end{array}$ & $\begin{array}{l}15.3 \\
22.8 \\
30.4 \\
12.3\end{array}$ & $\begin{array}{l}-- \\
-- \\
--\end{array}$ & $\begin{array}{l}-- \\
-- \\
--\end{array}$ & $\begin{array}{l}-- \\
-- \\
--\end{array}$ & $\begin{array}{l}42.9 \\
28.7 \\
10.6 \\
40.6\end{array}$ & $\begin{array}{l}139.1 \\
141.1 \\
142.3 \\
144.9\end{array}$ & $\begin{array}{l}1,367.23 \\
1,412.83 \\
1,449.31 \\
1,481.29\end{array}$ \\
\hline 1863-WOM & 0.9 & 26.8 & -- & -- & -- & 16.7 & 145.8 & $1,505.41$ \\
\hline 1862-WOM & 1.0 & 19.0 & -- & -- & -- & 32.0 & 146.8 & $1,524.41$ \\
\hline
\end{tabular}




\begin{tabular}{|c|c|c|c|c|c|c|c|c|c|c|}
\hline \multirow{2}{*}{$\begin{array}{c}\text { Bed } \\
\text { no. }\end{array}$} & \multirow[b]{2}{*}{ Rock description } & \multirow{2}{*}{$\begin{array}{c}\text { Sample } \\
\text { no. }\end{array}$} & \multirow{2}{*}{$\begin{array}{c}\text { Thickness } \\
\text { (feet) }\end{array}$} & \multicolumn{5}{|c|}{ Chemical analyses (percent) } & \multirow{2}{*}{$\begin{array}{c}\text { Cumulative } \\
\text { thickness } \\
\text { (feet) }\end{array}$} & \multirow{2}{*}{$\begin{array}{l}\text { Thickness } \mathrm{x} \\
\text { percent } \mathrm{P}_{2} \mathrm{O}_{5} \\
\text { (cumulative) }\end{array}$} \\
\hline & & & & $\mathrm{P}_{2} \mathrm{O}_{5}$ & $\mathrm{Al}_{2} \mathrm{O}_{3}$ & $\mathrm{Fe}_{2} \mathrm{O}_{3}$ & $\begin{array}{l}\text { Loss on } \\
\text { ignition }\end{array}$ & $\begin{array}{l}\text { Acid } \\
\text { insoluble }\end{array}$ & & \\
\hline $\begin{array}{l}\text { P- } 19 \\
\text { P- } 18 \\
\text { P- } 17 \\
P-16 \\
P-15\end{array}$ & $\begin{array}{l}\text { Mudstone, phosphatic, and argillaceous } \\
\text { phosphate rock } \\
\text { Mudstone, phosphatic } \\
\text { Phosphate rock } \\
\text { Phosphate rock } \\
\text { Phosphate rock, argillaceous }\end{array}$ & $\begin{array}{l}1861-\text { WOM } \\
1860-\text { WOM } \\
1859-\text { WOM } \\
1858-\text { WOM } \\
1857-\text { WOM }\end{array}$ & $\begin{array}{l}1.6 \\
0.9 \\
0.9 \\
2.7 \\
0.4\end{array}$ & $\begin{array}{l}15.8 \\
15.2 \\
28.5 \\
32.0 \\
18.0\end{array}$ & $\begin{array}{l}-- \\
-- \\
3.1 \\
1.6 \\
5.4\end{array}$ & $\begin{array}{l}-- \\
1.1 \\
0.89 \\
2.4\end{array}$ & $\begin{array}{l}-- \\
-- \\
4.70 \\
5.60 \\
5.48\end{array}$ & $\begin{array}{l}49.0 \\
46.0 \\
19.7 \\
11.1 \\
42.7\end{array}$ & $\begin{array}{l}148.4 \\
149.3 \\
150.2 \\
152.9 \\
153.3\end{array}$ & $\begin{array}{l}1,549.69 \\
1,563.37 \\
1,589.02 \\
1,675.42 \\
1,682.62\end{array}$ \\
\hline $\begin{array}{l}P-14 \\
P-13 \\
P-12 \\
P=11 \\
P=10\end{array}$ & $\begin{array}{l}\text { Phosphate rock } \\
\text { Phosphate rock } \\
\text { Phosphate rock, argillaceous } \\
\text { Phosphate rock } \\
\text { Phosphate rock, calcareous }\end{array}$ & $\begin{array}{l}1856-\text { WOM } \\
1855-\text { WOM } \\
1854-\text { WOM } \\
1853-\text { WOM } \\
1852-\text { WOM }\end{array}$ & $\begin{array}{l}2.4 \\
3.0 \\
0.3 \\
1.1 \\
0.5\end{array}$ & $\begin{array}{l}34.5 \\
34.0 \\
17.3 \\
29.6 \\
19.3\end{array}$ & $\begin{array}{l}0.85 \\
3.2 \\
7.8 \\
2.0 \\
6.1\end{array}$ & $\begin{array}{l}0.61 \\
0.60 \\
2.0 \\
0.96 \\
2.8\end{array}$ & $\begin{array}{l}4.38 \\
3.12 \\
6.46 \\
5.36 \\
9.78\end{array}$ & $\begin{array}{r}5.8 \\
6.9 \\
43.7 \\
16.0 \\
3.3\end{array}$ & $\begin{array}{l}155.7 \\
158.7 \\
159.0 \\
160.1 \\
160.6\end{array}$ & $\begin{array}{l}1,765.42 \\
1,867.42 \\
1,872.61 \\
1,905.17 \\
1,914.82\end{array}$ \\
\hline $\begin{array}{l}\text { P- } \\
\text { P- }\end{array}$ & $\begin{array}{l}\text { Phosphate rock } \\
\text { Phosphate rock, argillaceous; fos. }\end{array}$ & $1851-W O M$ & 2.0 & 28.3 & 3.1 & 1.0 & 7.64 & 15.4 & 162.6 & $1,971.42$ \\
\hline $\begin{array}{ll}\text { P- } & 7 \\
\text { P- } & 6 \\
\text { P- } & 5\end{array}$ & $\begin{array}{l}\text { col. no. } 48-J E S-136 \\
\text { Phosphate rock } \\
\text { Phosphate rock } \\
\text { Phosphate rock }\end{array}$ & $\begin{array}{l}1840-\text { WOM } \\
1839-\text { WOM } \\
1838-\text { WOM } \\
1837-\text { WOM }\end{array}$ & $\begin{array}{l}0.5 \\
2.6 \\
0.9 \\
2.0\end{array}$ & $\begin{array}{l}20.4 \\
28.6 \\
26.7 \\
34.0\end{array}$ & $\begin{array}{l}5.8 \\
2.5 \\
2.5 \\
0.71\end{array}$ & $\begin{array}{l}2.1 \\
0.89 \\
1.3 \\
0.48\end{array}$ & $\begin{array}{r}11.06 \\
7.28 \\
6.18 \\
5.92\end{array}$ & $\begin{array}{r}28.1 \\
14.7 \\
19.4 \\
3.1\end{array}$ & $\begin{array}{l}163.1 \\
165.7 \\
166.6 \\
168.6\end{array}$ & $\begin{array}{l}1,981.62 \\
2,055.98 \\
2,080.01 \\
2,148.01\end{array}$ \\
\hline $\begin{array}{ll}\text { P- } & 4 \\
\text { P- } & 3 \\
\text { P- } & 2 \\
\text { P- } & 1\end{array}$ & $\begin{array}{l}\text { Phosphate rock } \\
\text { Mudstone } \\
\text { Mudstone } \\
\text { Phosphate rock }\end{array}$ & $\begin{array}{l}1836-\text { WOM } \\
1835-\text { WOM } \\
1834-\text { WOM } \\
1833-\text { WOM }\end{array}$ & $\begin{array}{l}3.5 \\
0.7 \\
2.0 \\
0.3\end{array}$ & $\begin{array}{r}30.6 \\
1.8 \\
0.8 \\
33.7\end{array}$ & $\begin{array}{l}1.9 \\
7.9 \\
8.7 \\
0.85\end{array}$ & $\begin{array}{l}0.62 \\
3.5 \\
3.2 \\
0.59\end{array}$ & $\begin{array}{r}5.94 \\
11.92 \\
14.50 \\
3.82\end{array}$ & $\begin{array}{r}9.7 \\
66.7 \\
61.0 \\
6.0\end{array}$ & $\begin{array}{l}172.1 \\
172.8 \\
174.8 \\
175.1\end{array}$ & $\begin{array}{l}2,255.11 \\
2,256.37 \\
2,257.97 \\
2,268.08\end{array}$ \\
\hline \multicolumn{11}{|c|}{ Wells formation - top beds only } \\
\hline $\begin{array}{l}\mathrm{Cw}-1 \\
\mathrm{Cw}-2\end{array}$ & $\begin{array}{l}\text { Limestone } \\
\text { Limestone }\end{array}$ & $\begin{array}{l}1832-\text { WOM } \\
1831-\text { WOM }\end{array}$ & $\begin{array}{l}2.4 \\
1.6\end{array}$ & $\begin{array}{l}0.7 \\
0.3\end{array}$ & -- & -- & -- & $\begin{array}{l}4.3 \\
3.4\end{array}$ & $\begin{array}{l}2.4 \\
4.0\end{array}$ & $\begin{array}{l}1.68 \\
2.16\end{array}$ \\
\hline
\end{tabular}


NORTH DRY VALLEY, IDAHO. LOT NO. 1259.

Part of phosphatic shale member of Phosphoria formation sampled in bulldozer trench on west slope of North Dry Valley about 100 feet above

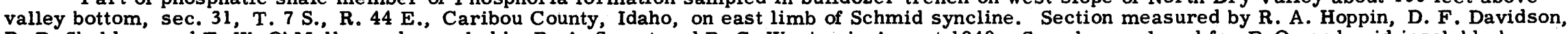

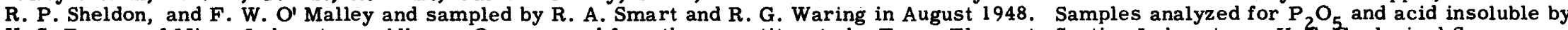
U. S. Bureau of Mines Laboratory, Albany, Oregon, and for other constituents by Trace Elements Section Laboratory, U. S. Geological Survey, Washington, D. C.

\begin{tabular}{|c|c|c|c|c|c|c|c|c|c|c|}
\hline \multirow{2}{*}{$\begin{array}{c}\text { Bed } \\
\text { no. }\end{array}$} & \multirow[b]{2}{*}{ Rock description } & \multirow{2}{*}{$\begin{array}{c}\text { Sample } \\
\text { no. }\end{array}$} & \multirow{2}{*}{$\begin{array}{c}\text { Thickness } \\
\text { (feet) }\end{array}$} & \multicolumn{5}{|c|}{ Chemical analyses (percent) } & \multirow{2}{*}{$\begin{array}{c}\text { Cumulative } \\
\text { thickness } \\
\text { (feet) }\end{array}$} & \multirow{2}{*}{$\begin{array}{l}\text { Thickness } \mathrm{x} \\
\text { percent } \mathrm{P}_{2} \mathrm{O}_{5} \\
\text { (cumulative) }\end{array}$} \\
\hline & & & & $\mathrm{P}_{2} \mathrm{O}_{5}$ & $\mathrm{Al}_{2} \mathrm{O}_{3}$ & $\mathrm{Fe}_{2} \mathrm{O}_{3}$ & $\begin{array}{l}\text { Loss on } \\
\text { ignition }\end{array}$ & $\begin{array}{c}\text { Acid } \\
\text { insoluble }\end{array}$ & & \\
\hline
\end{tabular}

Rex member of Phosphoria formation-basal beds only

\begin{tabular}{|c|c|c|c|c|c|c|c|c|c|c|c|}
\hline $\begin{array}{ll}R- & 4 \\
R- & 3 \\
R- & 2 \\
R- & 1\end{array}$ & $\begin{array}{l}\text { Chert } \\
\text { Chert, argillaceous } \\
\text { Chert, argillaceous } \\
\text { Chert }\end{array}$ & - & $\begin{array}{l}1697-W O M \\
1696-W O M \\
1695-W O M \\
1694-W O M\end{array}$ & $\begin{array}{l}7.0 \\
1.2 \\
2.5 \\
1.9\end{array}$ & $\begin{array}{l}0.3 \\
0.2 \\
0.1 \\
1.3\end{array}$ & $\begin{array}{l}-- \\
-- \\
--\end{array}$ & $\begin{array}{l}-- \\
-- \\
--\end{array}$ & $\begin{array}{l}-- \\
-- \\
-- \\
--\end{array}$ & $\begin{array}{l}94.7 \\
92.3 \\
93.7 \\
89.6\end{array}$ & $\begin{array}{r}7.0 \\
8.2 \\
10.7 \\
12.6\end{array}$ & $\begin{array}{l}2.10 \\
2.34 \\
2.59 \\
5.06\end{array}$ \\
\hline
\end{tabular}

Phosphatic shale member of Phosphoria formation-base not exposed

\begin{tabular}{|c|c|c|c|c|c|c|c|c|c|c|}
\hline $\begin{array}{l}P-101 \\
P-100 \\
P-99 \\
P-98 \\
P-97\end{array}$ & $\begin{array}{l}\text { Mudstone; fos. col. no. } 48 \text {-JES- } 164 \\
\text { Mudstone; fos, col. no. } 48 \text {-JES- } 163 \\
\text { Phosphate rock, argillaceous } \\
\text { Mudstone; fos, col. no. } 48 \text {-JES- } 162 \\
\text { Phosphate rock }\end{array}$ & $\begin{array}{l}1693-\text { WOM } \\
1692-\text { WOM } \\
1691-\text { WOM } \\
1690-\text { WOM } \\
1689-\text { WOM }\end{array}$ & $\begin{array}{l}4.8 \\
5.0 \\
0.8 \\
4.5 \\
0.5\end{array}$ & $\begin{array}{r}1.6 \\
2.6 \\
18.4 \\
1.6 \\
35.3\end{array}$ & $\begin{array}{l}-- \\
-- \\
-- \\
-- \\
1.6\end{array}$ & $\begin{array}{l}-- \\
-- \\
-- \\
-- \\
0.56\end{array}$ & $\begin{array}{l}-- \\
-- \\
-- \\
-- \\
2.74\end{array}$ & $\begin{array}{r}73.6 \\
71.8 \\
41.4 \\
75.3 \\
-6.6\end{array}$ & $\begin{array}{r}4.8 \\
9.8 \\
10.6 \\
15.1 \\
15.6\end{array}$ & $\begin{array}{r}7.68 \\
20.68 \\
35.40 \\
42.60 \\
60.25\end{array}$ \\
\hline $\begin{array}{l}P-96 \\
P-95 \\
P=94 \\
P=93 \\
P-92\end{array}$ & $\begin{array}{l}\text { Phosphate rock, argillaceous } \\
\text { Phosphate rock } \\
\text { Mudstone } \\
\text { Phosphate rock } \\
\text { Mudstone, phosphatic }\end{array}$ & $\begin{array}{l}1688-\text { WOM } \\
1687-\text { WOM } \\
1686-\text { WOM } \\
1685-\text { WOM } \\
1684-\text { WOM }\end{array}$ & $\begin{array}{l}0.5 \\
0.6 \\
0.4 \\
0.7 \\
0.5\end{array}$ & $\begin{array}{r}18.6 \\
35.1 \\
7.5 \\
33.2 \\
10.7\end{array}$ & $\begin{array}{l}5.8 \\
1.5 \\
9.3 \\
1.5 \\
8.2\end{array}$ & $\begin{array}{l}2.33 \\
0.68 \\
2.84 \\
0.69 \\
2.78\end{array}$ & $\begin{array}{l}4.08 \\
2.74 \\
4.68 \\
8.70 \\
5.70\end{array}$ & $\begin{array}{r}41.6 \\
6.6 \\
65.6 \\
9.4 \\
57.1\end{array}$ & $\begin{array}{l}16.1 \\
16.7 \\
17.1 \\
17.8 \\
18.3\end{array}$ & $\begin{array}{r}69.55 \\
90.61 \\
93.61 \\
116.85 \\
122.20\end{array}$ \\
\hline $\begin{array}{l}P-91 \\
P-90 \\
P-89\end{array}$ & $\begin{array}{l}\text { Phosphate rock } \\
\text { Phosphate rock } \\
\text { Mudstone, phosphatic; fos. col. no. }\end{array}$ & $\begin{array}{l}1683-\text { WOM } \\
1682-\text { WOM }\end{array}$ & $\begin{array}{l}0.7 \\
1.2\end{array}$ & $\begin{array}{l}34.2 \\
29.5\end{array}$ & $\begin{array}{l}1.4 \\
3.3\end{array}$ & $\begin{array}{l}0.58 \\
1.12\end{array}$ & $\begin{array}{l}3.18 \\
3.52\end{array}$ & $\begin{array}{r}7.9 \\
17.3\end{array}$ & $\begin{array}{l}19.0 \\
20.2\end{array}$ & $\begin{array}{l}146.14 \\
181.54\end{array}$ \\
\hline $\begin{array}{l}P=88 \\
P=87\end{array}$ & $\begin{array}{l}\text { 48-JES- } 161 \\
\text { Phosphate rock } \\
\text { Mudstone, phosphatic }\end{array}$ & $\begin{array}{l}1681-\text { WOM } \\
1661-\text { WOM } \\
1660-\text { WOM }\end{array}$ & $\begin{array}{l}0.6 \\
0.4 \\
0.9\end{array}$ & $\begin{array}{r}9.1 \\
33.3 \\
9.8\end{array}$ & $\begin{array}{l}9.8 \\
1.6 \\
8.2\end{array}$ & $\begin{array}{l}3.58 \\
0.83 \\
3.17\end{array}$ & $\begin{array}{l}6.14 \\
3.46 \\
6.50\end{array}$ & $\begin{array}{r}57.6 \\
9.2 \\
58.0\end{array}$ & $\begin{array}{l}20.8 \\
21.2 \\
22.1\end{array}$ & $\begin{array}{l}187.00 \\
200.32 \\
209.14\end{array}$ \\
\hline $\begin{array}{l}P-86 \\
P-85 \\
P-84 \\
P-83\end{array}$ & $\begin{array}{l}\text { Phosphate rock } \\
\text { Phosphate rock } \\
\text { Phosphate rock; fos. col. no. } 48 \text {-JES- } 160 \text {. } \\
\text { Phosphate rock, argillaceous; fos. col. }\end{array}$ & $\begin{array}{l}1659-\text { WOM } \\
1658-\text { WOM } \\
1657-\text { WOM }\end{array}$ & $\begin{array}{l}0.6 \\
2.4 \\
4.4\end{array}$ & $\begin{array}{l}33.5 \\
34.1 \\
29.4\end{array}$ & $\begin{array}{l}2.2 \\
2.0 \\
3.3\end{array}$ & $\begin{array}{l}0.98 \\
0.76 \\
1.36\end{array}$ & $\begin{array}{l}3.90 \\
3.78 \\
5.98\end{array}$ & $\begin{array}{r}8.3 \\
7.4 \\
15.0\end{array}$ & $\begin{array}{l}22.7 \\
25.1 \\
29.5\end{array}$ & $\begin{array}{l}229.24 \\
311.08 \\
440.44\end{array}$ \\
\hline P- 82 & $\begin{array}{l}\text { no. 48-JES-159 } \\
\text { Phosphate rock, argillaceous; fos. col. } \\
\text { no. 48-JES-158 }\end{array}$ & $\begin{array}{l}1656-\text { WOM } \\
1655-\text { RPS }\end{array}$ & $\begin{array}{l}3.4 \\
2.6\end{array}$ & $\begin{array}{l}23.4 \\
17.9\end{array}$ & $\begin{array}{l}4.6 \\
--\end{array}$ & $\begin{array}{c}2.23 \\
--\end{array}$ & $\begin{array}{c}7.98 \\
--\end{array}$ & $\begin{array}{l}25.6 \\
34.5\end{array}$ & $\begin{array}{l}32.9 \\
35.5\end{array}$ & $\begin{array}{l}520.00 \\
566.54\end{array}$ \\
\hline P- 81 & Mudstone, phosphatic & 1654- RPS & 0.5 & 12.3 & -- & -- & -- & 47.8 & 36.0 & 572.69 \\
\hline
\end{tabular}

1 Fossil collection made by J. E. Smedley, Paleontology and Stratigraphy Branch, U. S. Geological Survey. 


\begin{tabular}{|c|c|c|c|c|c|c|c|c|c|c|}
\hline \multirow[b]{2}{*}{$\begin{array}{c}\text { Bed } \\
\text { no. }\end{array}$} & \multirow[b]{2}{*}{ Rock description } & \multirow[b]{2}{*}{$\begin{array}{l}\text { Sample } \\
\text { no. }\end{array}$} & \multirow{2}{*}{$\begin{array}{c}\text { Thickness } \\
\text { (feet) }\end{array}$} & \multicolumn{5}{|c|}{ Chemical analyses (percent) } & \multirow{2}{*}{$\begin{array}{l}\text { Cumulative } \\
\text { thickness } \\
\text { (feet) }\end{array}$} & \multirow{2}{*}{$\begin{array}{l}\text { Thickness } x \\
\text { percent } \mathrm{P}_{2} \mathrm{O}_{5} \\
\text { (cumulative) }\end{array}$} \\
\hline & & & & $\mathrm{P}_{2} \mathrm{O}_{5}$ & $\mathrm{Al}_{2} \mathrm{O}_{3}$ & $\mathrm{Fe}_{2} \mathrm{O}_{3}$ & $\begin{array}{l}\text { Loss on } \\
\text { ignition }\end{array}$ & $\begin{array}{l}\text { Acid } \\
\text { insoluble }\end{array}$ & & \\
\hline $\begin{array}{l}P-80 \\
P-79 \\
P-78 \\
P-77 \\
P-76\end{array}$ & $\begin{array}{l}\text { Mudstone, phosphatic } \\
\text { Phosphate rock, argillaceous } \\
\text { Mudstone and phosphate rock } \\
\text { Mudstone } \\
\text { Mudstone }\end{array}$ & $\begin{array}{l}1653-\text { RPS } \\
1652-\text { RPS } \\
1651-\text { RPS } \\
1650-\text { RPS } \\
1700-\text { WOM }\end{array}$ & $\begin{array}{l}0.8 \\
0.7 \\
0.9 \\
1.0 \\
1.5\end{array}$ & $\begin{array}{r}8.3 \\
19.1 \\
12.4 \\
1.6 \\
18.8\end{array}$ & $\begin{array}{l}-- \\
-- \\
-- \\
--\end{array}$ & $\begin{array}{l}-- \\
-- \\
-- \\
--\end{array}$ & $\begin{array}{l}-- \\
-- \\
-- \\
--\end{array}$ & $\begin{array}{l}55.6 \\
37.3 \\
55.7 \\
79.7 \\
38.8\end{array}$ & $\begin{array}{l}36.8 \\
37.5 \\
38.4 \\
39.4 \\
40.9\end{array}$ & $\begin{array}{l}579.33 \\
592.70 \\
603.86 \\
605.46 \\
633.66\end{array}$ \\
\hline $\begin{array}{l}P-75 \\
P-74\end{array}$ & $\begin{array}{l}\text { Phosphate rock, argillaceous } \\
\text { Mudstone, phosphatic; fos. col. no. }\end{array}$ & 1699-WOM & .0 .4 & 24.0 & -- & -- & -- & 29.5 & 41.3 & 643.26 \\
\hline $\begin{array}{l}P-73 \\
P-72 \\
P-71\end{array}$ & $\begin{array}{l}\text { 48-JES-157 } \\
\text { Mudstone, phosphatic } \\
\text { Phosphate rock, argillaceous } \\
\text { Mudstone; fos. col. no. } 48-J E S-156\end{array}$ & $\begin{array}{l}1698-\text { WOM } \\
1720-\text { WOM } \\
1719-\text { WOM } \\
1718-\text { WOM }\end{array}$ & $\begin{array}{l}5.0 \\
1.5 \\
0.7 \\
3.3\end{array}$ & $\begin{array}{r}8.1 \\
13.0 \\
27.2 \\
5.1\end{array}$ & $\begin{array}{l}-- \\
-- \\
--\end{array}$ & $\begin{array}{l}-- \\
-- \\
--\end{array}$ & $\begin{array}{l}-- \\
-- \\
--\end{array}$ & $\begin{array}{l}67.4 \\
58.0 \\
23.4 \\
76.8\end{array}$ & $\begin{array}{l}46.3 \\
47.8 \\
48.5 \\
51.8\end{array}$ & $\begin{array}{l}683.76 \\
703.26 \\
722.30 \\
739.13\end{array}$ \\
\hline $\begin{array}{l}P-70 \\
P-69 \\
P-68 \\
P-67 \\
P-66\end{array}$ & $\begin{array}{l}\text { Mudstone, phosphatic } \\
\text { Mudstone; fos. col. no. } 48-J E S-155 \\
\text { Mudstone } \\
\text { Mudstone, phosphatic } \\
\text { Phosphate rock, argillaceous }\end{array}$ & $\begin{array}{l}1717-\text { WOM } \\
1716-\text { WOM } \\
1715-\text { WOM } \\
1714-\text { WOM } \\
1713-\text { WOM }\end{array}$ & $\begin{array}{l}2.8 \\
2.2 \\
1.0 \\
1.3 \\
0.7\end{array}$ & $\begin{array}{r}10.3 \\
5.7 \\
4.8 \\
8.6 \\
20.5\end{array}$ & $\begin{array}{l}-- \\
-- \\
-- \\
--\end{array}$ & $\begin{array}{l}-- \\
-- \\
-- \\
--\end{array}$ & $\begin{array}{l}-- \\
-- \\
-- \\
--\end{array}$ & $\begin{array}{l}62.6 \\
74.4 \\
72.7 \\
65.9 \\
39.0\end{array}$ & $\begin{array}{l}54.6 \\
56.8 \\
57.8 \\
59.1 \\
59.8\end{array}$ & $\begin{array}{l}767.97 \\
780.51 \\
785.31 \\
796.49 \\
810.84\end{array}$ \\
\hline $\begin{array}{l}P-65 \\
P-64 \\
P-63 \\
P-62 \\
P-61\end{array}$ & $\begin{array}{l}\text { Mudstone, phosphatic; fos. col. no. } \\
48-J E S-165 \\
\text { Mudstone } \\
\text { Mudstone } \\
\text { Phosphate rock, argillaceous } \\
\text { Mudstone; fos. col. no. } 48 \text {-JES- } 154\end{array}$ & $\begin{array}{l}1712-\text { WOM } \\
1711-\text { WOM } \\
1727-\text { DFD } \\
1726-\text { DFD } \\
1725-\text { DFD }\end{array}$ & $\begin{array}{l}3.2 \\
0.4 \\
1.3 \\
0.6 \\
0.7\end{array}$ & $\begin{array}{r}8.7 \\
4.5 \\
6.2 \\
24.1 \\
5.8\end{array}$ & $\begin{array}{l}-- \\
-- \\
-- \\
--\end{array}$ & $\begin{array}{l}-- \\
-- \\
-- \\
--\end{array}$ & $\begin{array}{l}-- \\
-- \\
-- \\
--\end{array}$ & $\begin{array}{l}63.0 \\
66.4 \\
70.7 \\
28.0 \\
71.2\end{array}$ & $\begin{array}{l}63.0 \\
63.4 \\
64.7 \\
65.3 \\
66.0\end{array}$ & $\begin{array}{l}838.68 \\
840.48 \\
848.54 \\
863.00 \\
867.06\end{array}$ \\
\hline $\begin{array}{l}P-60 \\
P-59 \\
P-58 \\
P-57 \\
P-56\end{array}$ & $\begin{array}{l}\text { Mudstone, phosphatic } \\
\text { Phosphate rock, argillaceous } \\
\text { Mudstone, phosphatic } \\
\text { Mudstone, phosphatic } \\
\text { Mudstone }\end{array}$ & $\begin{array}{l}1724-\text { DFD } \\
1723-\text { DFD } \\
1722-\text { DFD } \\
1721-\text { DFD } \\
1710-\text { DFD }\end{array}$ & $\begin{array}{l}1.6 \\
1.1 \\
0.6 \\
0.5 \\
3.7\end{array}$ & $\begin{array}{r}12.3 \\
18.5 \\
9.8 \\
11.3 \\
4.5\end{array}$ & $\begin{array}{l}-- \\
-- \\
-- \\
-- \\
--\end{array}$ & $\begin{array}{l}-- \\
-- \\
-- \\
--\end{array}$ & $\begin{array}{l}-- \\
-- \\
-- \\
-- \\
--\end{array}$ & $\begin{array}{l}51.0 \\
37.7 \\
58.8 \\
56.3 \\
76.8\end{array}$ & $\begin{array}{l}67.6 \\
68.7 \\
69.3 \\
69.8 \\
73.5\end{array}$ & $\begin{array}{l}886.74 \\
907.09 \\
912.97 \\
918.62 \\
935.27\end{array}$ \\
\hline $\begin{array}{l}P-55 \\
P-54 \\
P-53 \\
P-52 \\
P-51\end{array}$ & $\begin{array}{l}\text { Mudstone } \\
\text { Mudstone } \\
\text { Mudstone } \\
\text { Mudstone } \\
\text { Mudstone, phosphatic }\end{array}$ & $\begin{array}{l}1709-\text { DFD } \\
1708-\text { DFD } \\
1707-\text { DFD } \\
1706-\text { DFD } \\
1705-\text { DFD }\end{array}$ & $\begin{array}{l}4.2 \\
1.7 \\
2.0 \\
3.1 \\
0.8\end{array}$ & $\begin{array}{r}2.0 \\
1.7 \\
3.3 \\
1.5 \\
10.9\end{array}$ & $\begin{array}{l}-- \\
-- \\
-- \\
--\end{array}$ & $\begin{array}{l}-- \\
-- \\
-- \\
--\end{array}$ & $\begin{array}{l}-- \\
-- \\
-- \\
--\end{array}$ & $\begin{array}{l}78.5 \\
81.5 \\
81.3 \\
82.0 \\
60.5\end{array}$ & $\begin{array}{l}77.7 \\
79.4 \\
81.4 \\
84.5 \\
85.3\end{array}$ & $\begin{array}{l}943.67 \\
946.56 \\
953.16 \\
957.81 \\
966.53\end{array}$ \\
\hline $\begin{array}{l}P-50 \\
P-49 \\
P-48 \\
P-47 \\
P-46\end{array}$ & $\begin{array}{l}\text { Mudstone } \\
\text { Limestone, argillaceous } \\
\text { Mudstone; fos. col. no. } 48 \text {-JES-153 } \\
\text { Mudstone } \\
\text { Phosphate rock }\end{array}$ & $\begin{array}{l}1704-\text { DFD } \\
1703-\text { DFD } \\
1702-\text { DFD } \\
1701-\text { DFD } \\
1680-\text { DFD }\end{array}$ & $\begin{array}{l}1.0 \\
1.4 \\
1.4 \\
1.4 \\
1.1\end{array}$ & $\begin{array}{r}4.9 \\
3.9 \\
4.7 \\
3.6 \\
32.0\end{array}$ & $\begin{array}{l}-- \\
-- \\
-- \\
--\end{array}$ & $\begin{array}{l}-- \\
-- \\
-- \\
--\end{array}$ & $\begin{array}{l}-- \\
-- \\
-- \\
--\end{array}$ & $\begin{array}{l}78.3 \\
29.7 \\
76.3 \\
77.9 \\
13.2\end{array}$ & $\begin{array}{l}86.3 \\
87.7 \\
89.1 \\
90.5 \\
91.6\end{array}$ & $\begin{array}{r}971.43 \\
976.89 \\
983.47 \\
988.51 \\
1,023.71\end{array}$ \\
\hline $\begin{array}{l}P-45 \\
P-44 \\
P-43 \\
P-42\end{array}$ & $\begin{array}{l}\text { Mudstone, phosphatic } \\
\text { Mudstone } \\
\text { Mudstone } \\
\text { Mudstone, phosphatic }\end{array}$ & $\begin{array}{l}1679-\text { DFD } \\
1678-\text { DFD } \\
1677-\text { DFD } \\
1676-\text { DFD }\end{array}$ & $\begin{array}{l}1.1 \\
1.7 \\
1.2 \\
0.9\end{array}$ & $\begin{array}{r}15.8 \\
4.2 \\
4.4 \\
10.1\end{array}$ & $\begin{array}{l}-- \\
-- \\
--\end{array}$ & $\begin{array}{l}-- \\
-- \\
--\end{array}$ & $\begin{array}{l}-- \\
-- \\
-- \\
--\end{array}$ & $\begin{array}{l}45.8 \\
76.5 \\
71.8 \\
57.8\end{array}$ & $\begin{array}{l}92.7 \\
94.4 \\
95.6 \\
96.5\end{array}$ & $\begin{array}{l}1,041.09 \\
1,048.23 \\
1,053.51 \\
1,062.60\end{array}$ \\
\hline
\end{tabular}




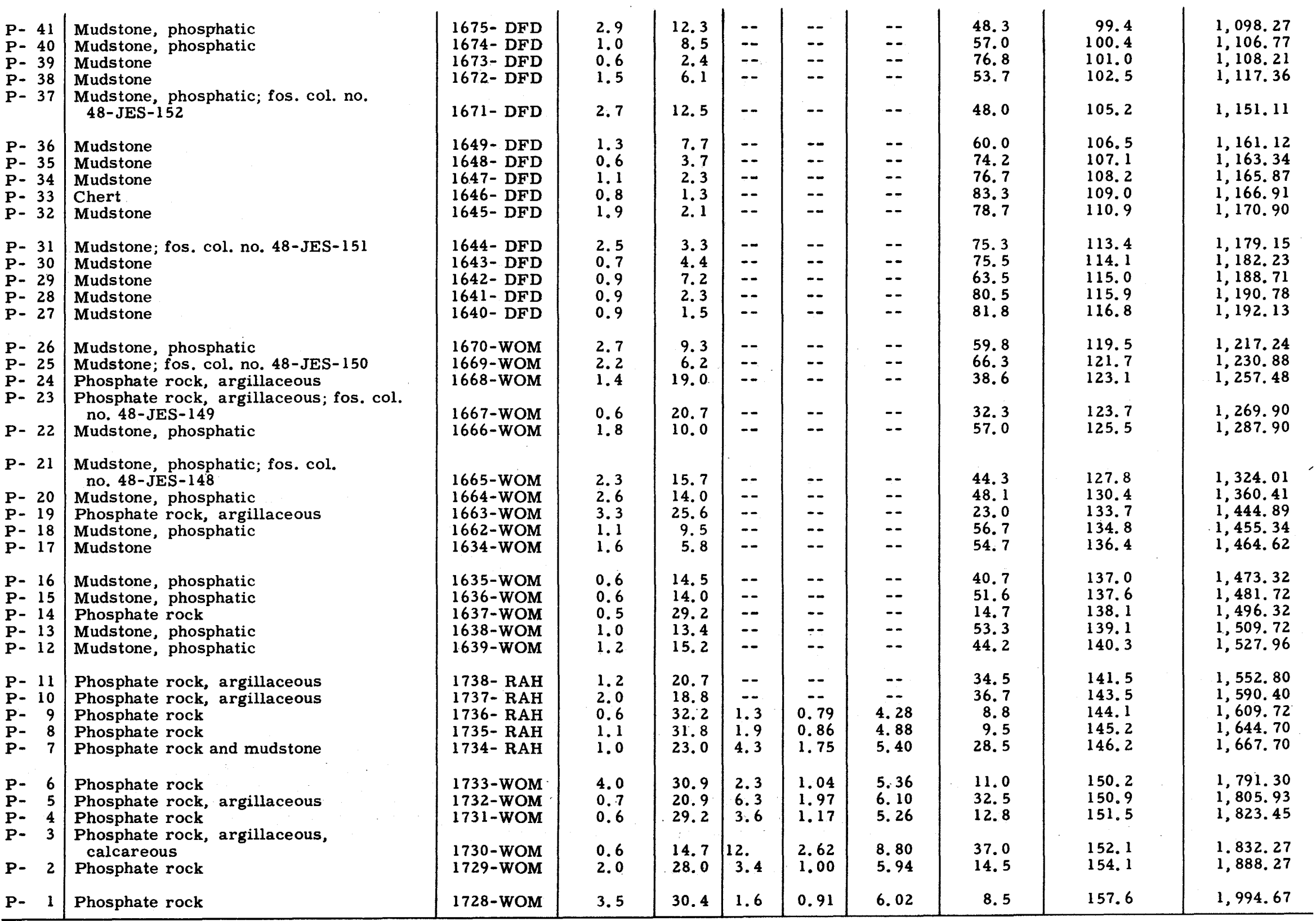


KENDALL CANYON, IDAHO. LOT NO. 1258.

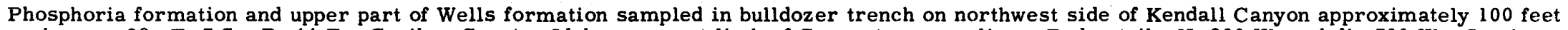

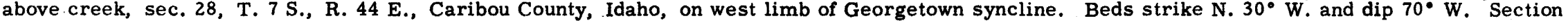

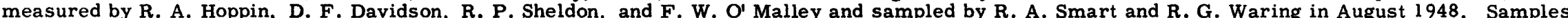

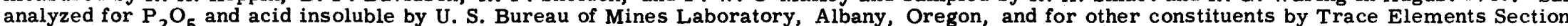
Laboratory, U. S. Geological Survey, Washington, D. C.

\begin{tabular}{|c|c|c|c|c|c|c|c|c|c|c|}
\hline \multirow{2}{*}{$\begin{array}{l}\text { Bed } \\
\text { no. }\end{array}$} & \multirow[b]{2}{*}{ Rock description } & \multirow{2}{*}{$\begin{array}{c}\text { Sample } \\
\text { no. }\end{array}$} & \multirow{2}{*}{$\begin{array}{c}\text { Thickness } \\
\text { (feet) }\end{array}$} & \multicolumn{5}{|c|}{ Chemical analyses (percent) } & \multirow{2}{*}{$\begin{array}{c}\text { Cumulative } \\
\text { thickness } \\
\text { (feet) }\end{array}$} & \multirow{2}{*}{$\begin{array}{l}\text { Thickness } x \\
\text { percent } \mathrm{P}_{2} \mathrm{O}_{5} \\
\text { (cumulative) }\end{array}$} \\
\hline & & & & $\mathrm{P}_{2} \mathrm{O}_{5}$ & $\mathrm{Al}_{2} \mathrm{O}_{3}$ & $\mathrm{Fe}_{2} \mathrm{O}_{3}$ & $\begin{array}{l}\text { Loss on } \\
\text { ignition }\end{array}$ & $\begin{array}{c}\text { Acid } \\
\text { insoluble }\end{array}$ & & \\
\hline
\end{tabular}

Rex member of Phosphoria formation-top not exposed

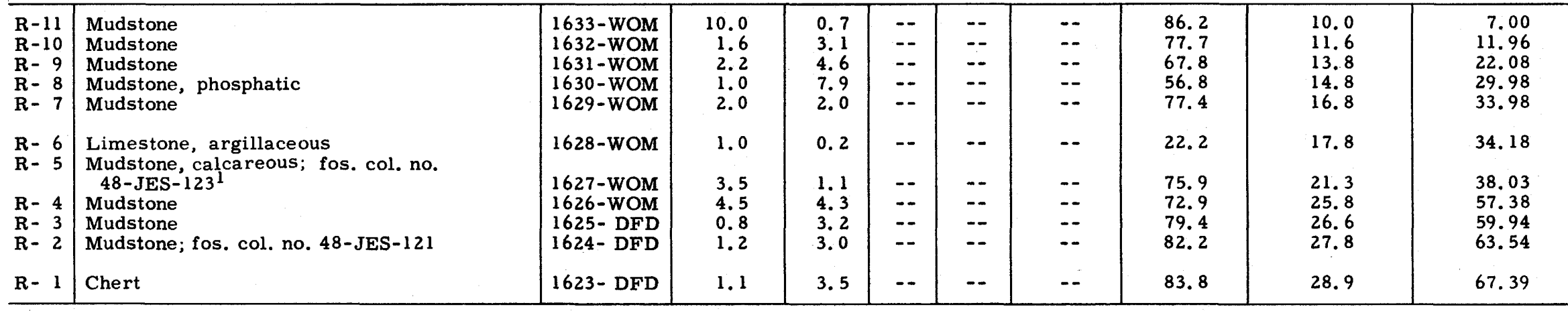

Phosphatic shale member of Phosphoria formation

Col. no. 48-JES-122 48 -JES-120

P-98 Mudstone; fos. col. no. 48-JES- 120

P-97 Mudstone; fos. col. no. 48-JES-119

P-96 Mudstone; fos. col. no. 48-JES-118

P-95 Mudstone

P-94 Phosphate rock

P-93 Mudstone, phosphatic

P-92 Mudstone

P-91 Phosphate rock and mudstone

P-90 Phosphate rock

P-89 Phosphate rock

P-88 Mudstone, phosphatic

P-87 Phosphate rock

P-86 Phosphate rock

P-85 Phosphate rock, argillaceous

P-84 Mudstone, phosphatic

\begin{tabular}{|l|l|r|r|r|r|r|r|r} 
1622- DFD & 0.5 & 22.7 & -- & -- & -- & 36.6 & 0.5 & 11.35 \\
$1621-$ DFD & 1.8 & 2.5 & -- & -- & -- & 76.8 & 2.3 & 15.85 \\
$1620-$ DFD & 0.5 & 1.5 & -- & -- & -- & 82.3 & 2.8 & 16.60 \\
$1619-$ DFD & 0.8 & 1.6 & -- & -- & -- & 82.6 & 3.6 & 17.88 \\
$1618-$ DFD & 1.3 & 1.2 & -- & -- & -- & 80.5 & 4.9 & 19.44 \\
$1617-$ DFD & 1.2 & 36.3 & 0.61 & 0.32 & 10.22 & 3.3 & 6.1 & 63.00 \\
$1616-$ DFD & 1.2 & 9.5 & 7.7 & 3.09 & 5.42 & 65.1 & 7.3 & 74.40 \\
$1615-$ DFD & 0.5 & 2.1 & 11. & 3.67 & 4.34 & 82.9 & 7.8 & 75.45 \\
$1614-$ DFD & 0.5 & 28.5 & 1.0 & 0.68 & 4.02 & 21.1 & 8.3 & 89.70 \\
$1613-$ DFD & 0.6 & 34.6 & 3.2 & 1.71 & 3.46 & 7.5 & 8.9 & 110.46 \\
$1612-$ DFD & 0.8 & 30.6 & 2.6 & 1.15 & 4.60 & 16.6 & 9.7 & 134.94 \\
$1609-$ WOM & 0.8 & 9.25 & 7.4 & 2.61 & 5.78 & 62.9 & 10.5 & 142.34 \\
$1608-$ WOM & 0.5 & 35.8 & 1.9 & 0.85 & 7.92 & 2.8 & 11.0 & 160.24 \\
$1607-$ WOM & 0.8 & 30.6 & 0.68 & 0.38 & 6.30 & 12.5 & 11.8 & 184.72 \\
$1606-$ WOM & 1.7 & 23.8 & 2.9 & 1.50 & 1.26 & 28.5 & 13.5 & 225.18 \\
$1605-$ WOM & 1.0 & 15.8 & 6.3 & 2.49 & 7.96 & 45.5 & 14.5 & 240.98 \\
1160 & & & & & & &
\end{tabular}




\begin{tabular}{|c|c|}
\hline $\begin{array}{l}P-83 \\
P-82 \\
P-81 \\
P-80 \\
P=79\end{array}$ & $\begin{array}{l}\text { Phosphate rock } \\
\text { Phosphate rock } \\
\text { Phosphate rock } \\
\text { Phosphate rock } \\
\text { Phosphate rock, argillaceous; fos. col. } \\
\text { no. } 48 \text {-JES-117 }\end{array}$ \\
\hline$P-78$ & $\begin{array}{l}\text { Mudstone, phosphatic; fos. col. no. } \\
\text { 48-JES-116 }\end{array}$ \\
\hline$P-77$ & Mudstone \\
\hline$P-76$ & Mudstone \\
\hline $\begin{array}{l}P-75 \\
P-74\end{array}$ & $\begin{array}{l}\text { Mudstone, phosphatic } \\
\text { Phosphate rock, argillaceous }\end{array}$ \\
\hline$P-73$ & $\begin{array}{l}\text { Mudstone and argillaceous phosphate } \\
\text { rock; fos. col. no. 48-JES-115 }\end{array}$ \\
\hline $\begin{array}{l}P-72 \\
P=71 \\
P-70 \\
P-69\end{array}$ & $\begin{array}{l}\text { Phosphate rock, a rgillaceous } \\
\text { Mudstone; fos. col. no. } 48-\mathrm{JES}-114 \\
\text { Mudstone, phosphatic } \\
\text { Mudstone; fos. col. no. } 48-\mathrm{JES}-113\end{array}$ \\
\hline $\begin{array}{l}P-68 \\
P-67 \\
P-66 \\
P-65 \\
P-64\end{array}$ & $\begin{array}{l}\text { Mudstone } \\
\text { Mudstone, phosphatic } \\
\text { Mudstone, phosphatic } \\
\text { Mudstone } \\
\text { Mudstone, phosphatic }\end{array}$ \\
\hline $\begin{array}{l}P-63 \\
P-62\end{array}$ & $\begin{array}{l}\text { Limestone, argillaceous } \\
\text { Mudstone and argillaceous phosphate }\end{array}$ \\
\hline $\begin{array}{l}P-61 \\
P-60 \\
P-59\end{array}$ & $\begin{array}{l}\text { Mudstone; fos. col. no. 48-JES-111 } \\
\text { Mudstone } \\
\text { Mudstone, phosphatic }\end{array}$ \\
\hline$P-58$ & $\begin{array}{l}\text { Phosphate rock, argillaceous; fos, col. } \\
\text { no. 48-JES-110 }\end{array}$ \\
\hline $\begin{array}{l}P-57 \\
P-56 \\
P-55 \\
P-54\end{array}$ & $\begin{array}{l}\text { Mudstone, phosphatic } \\
\text { Mudstone } \\
\text { Mudstone } \\
\text { Mudstone }\end{array}$ \\
\hline $\begin{array}{l}P-53 \\
P-52 \\
P-51 \\
P-50 \\
P-49\end{array}$ & $\begin{array}{l}\text { Phosphate rock, argillaceous } \\
\text { Mudstone } \\
\text { Phosphate rock } \\
\text { Mudstone } \\
\text { Phosphate rock, argillaceous }\end{array}$ \\
\hline $\begin{array}{l}P-48 \\
P-47\end{array}$ & $\begin{array}{l}\text { Mudstone; fos. col. no. 48-JES-109 } \\
\text { Mudstone }\end{array}$ \\
\hline
\end{tabular}

\begin{tabular}{|l|}
$1604-$ WOM \\
$1603-$ WOM \\
$1602-$ WOM \\
$1601-$ WOM \\
$1600-$ WOM \\
\\
$1579-$ RAH \\
$1578-$ RAH \\
$1577-$ RAH \\
$1599-$ WOM \\
$1598-$ WOM \\
$1597-$ WOM \\
$1596-$ WOM \\
$1595-$ WOM \\
$1594-$ WOM \\
$1593-$ WOM \\
$1592-$ WOM \\
$1591-$ WOM \\
$1590-$ WOM \\
$1589-$ WOM \\
$1588-$ WOM \\
$1587-$ WOM \\
$1586-$ WOM \\
$1585-$ WOM \\
$1611-$ DFD \\
$1569-$ DFD \\
$1568-$ DFD \\
$1567-$ DFD \\
$1566-$ DFD \\
$1565-$ DFD \\
$1584-$ WOM \\
$1583-$ WOM \\
$1564-$ DFD \\
$1610-$ DFD \\
$1563-$ DFD \\
$1562-$ DFD \\
$1561-$ DFD \\
$1560-$ DFD
\end{tabular}

\begin{tabular}{|c|c|c|c|}
\hline $\begin{array}{l}0.6 \\
2.0 \\
3.4 \\
2.2\end{array}$ & $\begin{array}{l}37.1 \\
33.1 \\
25.4 \\
24.7\end{array}$ & $\begin{array}{l}0.51 \\
0.96 \\
3.3 \\
3.0\end{array}$ & $\begin{array}{l}0.51 \\
0.51 \\
1.48 \\
1.35\end{array}$ \\
\hline 2.4 & 21.8 & 5.3 & 1.67 \\
\hline $\begin{array}{l}4.2 \\
1.2 \\
1.3 \\
2.3 \\
0.5\end{array}$ & $\begin{array}{r}9.7 \\
7.4 \\
2.1 \\
14.3 \\
16.2\end{array}$ & $\begin{array}{l}-- \\
-- \\
-- \\
--\end{array}$ & $\begin{array}{l}-- \\
-- \\
-- \\
--\end{array}$ \\
\hline $\begin{array}{l}5.0 \\
2.0 \\
3.7 \\
1.8 \\
3.0\end{array}$ & $\begin{array}{r}9.7 \\
19.2 \\
6.4 \\
12.7 \\
7.7\end{array}$ & $\begin{array}{l}-- \\
-- \\
-- \\
--\end{array}$ & $\begin{array}{l}-- \\
-- \\
-- \\
--\end{array}$ \\
\hline $\begin{array}{l}1.0 \\
1.3 \\
1.0 \\
1.7 \\
1.0\end{array}$ & $\begin{array}{r}4.1 \\
10.6 \\
13.8 \\
7.2 \\
7.8\end{array}$ & $\begin{array}{l}-- \\
-- \\
-- \\
--\end{array}$ & $\begin{array}{l}-- \\
-- \\
-- \\
--\end{array}$ \\
\hline 0.8 & 4.1 & -- & -- \\
\hline $\begin{array}{l}1.5 \\
0.8 \\
1.0 \\
0.7\end{array}$ & $\begin{array}{r}12.3 \\
4.7 \\
7.2 \\
12.7\end{array}$ & $\begin{array}{l}-- \\
-- \\
-- \\
--\end{array}$ & $\begin{array}{l}-- \\
-- \\
-- \\
--\end{array}$ \\
\hline $\begin{array}{l}0.5 \\
1.0 \\
1.7 \\
1.2 \\
5.0\end{array}$ & $\begin{array}{r}23.8 \\
14.6 \\
1.5 \\
2.0 \\
2.1\end{array}$ & $\begin{array}{l}-- \\
-- \\
-- \\
--\end{array}$ & $\begin{array}{l}-- \\
-- \\
-- \\
-- \\
--\end{array}$ \\
\hline $\begin{array}{l}1.5 \\
1.1 \\
0.6 \\
4.7 \\
1.1\end{array}$ & $\begin{array}{r}22.3 \\
0.5 \\
28.5 \\
5.4 \\
19.8\end{array}$ & $\begin{array}{l}-- \\
-- \\
-- \\
--\end{array}$ & $\begin{array}{l}-- \\
-- \\
-- \\
--\end{array}$ \\
\hline $\begin{array}{l}2.5 \\
1.1\end{array}$ & $\begin{array}{l}3.0 \\
0.9\end{array}$ & $=-$ & $=$ \\
\hline
\end{tabular}

\begin{tabular}{|c|c|}
\hline $\begin{array}{c}4.96 \\
6.94 \\
- \\
12.60\end{array}$ & $\begin{array}{r}2.0 \\
7.3 \\
19.3 \\
19.3\end{array}$ \\
\hline 13.26 & 27.1 \\
\hline $\begin{array}{l}-- \\
-- \\
-- \\
--\end{array}$ & $\begin{array}{l}54.3 \\
67.1 \\
81.3 \\
42.1 \\
32.7\end{array}$ \\
\hline $\begin{array}{l}-- \\
-- \\
-- \\
--\end{array}$ & $\begin{array}{l}40.6 \\
41.8 \\
74.0 \\
54.0 \\
69.6\end{array}$ \\
\hline $\begin{array}{l}-- \\
-- \\
-- \\
--\end{array}$ & $\begin{array}{l}77.7 \\
60.3 \\
46.8 \\
64.6 \\
63.5\end{array}$ \\
\hline-- & 25.3 \\
\hline $\begin{array}{l}-- \\
-- \\
--\end{array}$ & $\begin{array}{l}54.6 \\
74.1 \\
52.3 \\
40.7\end{array}$ \\
\hline $\begin{array}{l}-- \\
-- \\
-- \\
--\end{array}$ & $\begin{array}{l}23.0 \\
47.8 \\
87.4 \\
84.4 \\
81.7\end{array}$ \\
\hline $\begin{array}{l}-- \\
-- \\
-- \\
--\end{array}$ & $\begin{array}{l}35.0 \\
86.8 \\
19.5 \\
75.0 \\
35.3\end{array}$ \\
\hline-- & $\begin{array}{l}80.6 \\
98.0\end{array}$ \\
\hline
\end{tabular}

15.1
17.1
20.5
22.7
25.1

29.3
30.5
31.8
34.1
34.6

39.6
41.6
45.3
47.1
50.1
51.1
52.4
53.4
55.1
56.1
56.9
58.4
59.2
60.2
60.9
61.4
62.4
64.1
65.3
70.3
71.8
72.9
73.5
78.2
79.3
81.8
82.9

827.34

845.79

849.55

856. 75

865.64

877.54

892. 14

894.69

894.69
897.09

907.59

${ }^{1}$ Fossil collection made by J. E. Smedley, Paleontology and Stratigraphy Branch, U. S. Geological Survey.

941.04

941.59

958.69

984.07

$1,005.85$

$1,013.35$

$1,014.34$ 


\begin{tabular}{|c|c|c|c|c|c|c|c|c|c|c|}
\hline \multirow{2}{*}{$\begin{array}{c}\text { Bed } \\
\text { no. }\end{array}$} & \multirow[b]{2}{*}{ Rock description } & \multirow{2}{*}{$\begin{array}{l}\text { Sample } \\
\text { no. }\end{array}$} & \multirow{2}{*}{$\begin{array}{c}\text { Thickness } \\
\text { (feet) }\end{array}$} & \multicolumn{5}{|c|}{ Chemical analyses (percent) } & \multirow{2}{*}{$\begin{array}{l}\text { Cumulative } \\
\text { thickness } \\
\text { (feet) }\end{array}$} & \multirow{2}{*}{$\begin{array}{l}\text { Thickness } x \\
\text { percent } P_{2} O_{5} \\
\text { (cumulative) }\end{array}$} \\
\hline & & & & $\mathrm{P}_{2} \mathrm{O}_{3}$ & $\mathrm{Al}_{2} \mathrm{O}_{3}$ & $\mathrm{Fe}_{2} \mathrm{O}_{3}$ & $\begin{array}{l}\text { Loss on } \\
\text { ignition }\end{array}$ & $\begin{array}{c}\text { Acid } \\
\text { insoluble }\end{array}$ & & \\
\hline$P-46$ & Mudstone, phosphatic & $1582-W O M$ & 0.4 & 10.9 & -- & -- & -- & 57.1 & 83.3 & $1,018,70$ \\
\hline$P-45$ & $\begin{array}{l}\text { Phosphate rock, argillaceous, } \\
\text { calcareous }\end{array}$ & $1581-W O M$ & 1.5 & 17.5 & -- & & -- & & & \\
\hline P- 44 & Mudstone & $1580-$ WOM & 2.3 & 7.4 & -- & -- & -- & 59.5 & 87.1 & $1,061.97$ \\
\hline$P-43$ & $\begin{array}{l}\text { Mudstone, calcareous, phosphatic; } \\
\text { fos. col. no. } 48-J E S-108\end{array}$ & 1559-WOM & 4.5 & 7.9 & -- & -- & -- & 56.5 & 91.6 & \\
\hline P- 42 & Mudstone, calcareous, phosphatic & $1558-W O M$ & 2.5 & 8.6 & -- & -- & -- & 50.9 & 94.1 & $1,119.02$ \\
\hline & $\begin{array}{l}\text { Mudstone, calcareous; fos. col. no. } \\
\text { 48-JES-102 }\end{array}$ & 1557-WOM & 2.0 & 1.7 & -- & -- & -- & 58.2 & 96.1 & $1,122.42$ \\
\hline $\begin{array}{l}P-40 \\
P-39\end{array}$ & $\begin{array}{l}\text { Limestone; fos. col. no. } 48 \text {-JES- } 100 \\
\text { Mudstone; fos. col. no. } 48 \text {-JES-101 }\end{array}$ & $\begin{array}{l}1556-W O M \\
1555-W O M\end{array}$ & 3.7 & $\begin{array}{l}0.3 \\
2.3\end{array}$ & -- & -- & -- & $\begin{array}{l}11.2 \\
80.7\end{array}$ & $\begin{array}{r}99.8 \\
102.8\end{array}$ & $\begin{array}{l}1,123.53 \\
1,130.43\end{array}$ \\
\hline $\begin{array}{l}\text { P- } 38 \\
P-37\end{array}$ & $\begin{array}{l}\text { Mudstone } \\
\text { Mudstone, phosphatic; fos. col. no. } \\
\text { 48-JES-99 }\end{array}$ & $\begin{array}{l}1554-\text { WOM } \\
1553-W O M\end{array}$ & $\begin{array}{l}2.0 \\
1.8\end{array}$ & $\begin{array}{l}3.9 \\
8.1\end{array}$ & -- & -- & -- & $\begin{array}{l}73.0 \\
63.4\end{array}$ & $\begin{array}{l}104.8 \\
106.6\end{array}$ & $\begin{array}{l}1,138.23 \\
1,152.81\end{array}$ \\
\hline$P-36$ & Mudstone & 1552-WOM & 0.6 & 1.7 & -- & -- & -- & 86.3 & 107.2 & $1,153.83$ \\
\hline$P-35$ & $\begin{array}{l}\text { Mudstone, phosphatic; fos. col. no. } \\
\text { 48-JES-98 }\end{array}$ & & 3.5 & 8.0 & -- & -- & -- & 62.2 & 110.7 & $1,181.83$ \\
\hline P- 34 & $\begin{array}{l}\text { Mudstone, phosphatic; fos. col. no. } \\
\text { 48-JES-97 }\end{array}$ & $1550-W O M$ & 2.6 & 12.1 & -- & -- & -- & 52.6 & 113.3 & $1,213.29$ \\
\hline $\begin{array}{l}P-33 \\
P-32\end{array}$ & $\begin{array}{l}\text { Phosphate rock, argillaceous, } \\
\text { calcareous } \\
\text { Mudstone, phosphatic }\end{array}$ & $\begin{array}{l}\text { 1549- DFD } \\
1548-\text { DFD }\end{array}$ & $\begin{array}{l}0.6 \\
0.8\end{array}$ & $\begin{array}{r}16.8 \\
8.5\end{array}$ & -- & -- & -- & $\begin{array}{l}33.7 \\
65.3\end{array}$ & $\begin{array}{l}113.9 \\
114.7\end{array}$ & $\begin{array}{l}1,223.37 \\
1,230.17\end{array}$ \\
\hline $\begin{array}{l}P-31 \\
P-30 \\
P-29 \\
P-28 \\
P-27\end{array}$ & $\begin{array}{l}\text { Mudstone, phosphatic; fos. col. no. } \\
48-J E S-96 \\
\text { Phosphate rock, argillaceous } \\
\text { Mudstone and phosphate rock } \\
\text { Phosphate rock and mudstone } \\
\text { Mudstone, phosphatic }\end{array}$ & $\begin{array}{l}1547-\text { DFD } \\
1546-\text { DFD } \\
1545-\text { DFD } \\
1544-\text { DFD } \\
1576-\text { RAH }\end{array}$ & $\begin{array}{l}0.5 \\
3.3 \\
0.9 \\
0.7 \\
1.4\end{array}$ & $\begin{array}{r}9.3 \\
19.7 \\
26.2 \\
26.4 \\
10.2\end{array}$ & $\begin{array}{l}-- \\
-- \\
-- \\
--\end{array}$ & $\begin{array}{l}-- \\
-- \\
-- \\
--\end{array}$ & $\begin{array}{l}-- \\
-- \\
-- \\
--\end{array}$ & $\begin{array}{l}63.1 \\
32.4 \\
14.9 \\
16.7 \\
43.3\end{array}$ & $\begin{array}{l}115.2 \\
118.5 \\
119.4 \\
120.1 \\
121.5\end{array}$ & $\begin{array}{l}1,234.82 \\
1,299.83 \\
1,323.41 \\
1,341.89 \\
1,356.17\end{array}$ \\
\hline $\begin{array}{l}P-26 \\
P-25 \\
P-24 \\
P-23 \\
P-22\end{array}$ & $\begin{array}{l}\text { Phosphate rock } \\
\text { Mudstone, phosphatic } \\
\text { Phosphate rock } \\
\text { Mudstone, phosphatic } \\
\text { Mudstone, phosphatic }\end{array}$ & $\begin{array}{l}1575-\text { RAH } \\
1574-\text { RAH } \\
1573-\text { RAH } \\
1572-\text { RAH } \\
1571-\text { RAH }\end{array}$ & $\begin{array}{l}2.0 \\
1.5 \\
0.5 \\
1.1 \\
2.3\end{array}$ & $\begin{array}{r}27.4 \\
7.5 \\
31.7 \\
13.0 \\
13.7\end{array}$ & $\begin{array}{l}-- \\
-- \\
-- \\
--\end{array}$ & $\begin{array}{l}-- \\
-- \\
-- \\
--\end{array}$ & $\begin{array}{l}-- \\
-- \\
-- \\
--\end{array}$ & $\begin{array}{r}15.3 \\
42.9 \\
5.7 \\
47.7 \\
49.3\end{array}$ & $\begin{array}{l}123.5 \\
125.0 \\
125.5 \\
126.6 \\
128.9\end{array}$ & $\begin{array}{l}1,410.97 \\
1,422.22 \\
1,438.07 \\
1,452.37 \\
1,483.88\end{array}$ \\
\hline $\begin{array}{l}P-21 \\
P-20 \\
P=19 \\
P-18\end{array}$ & $\begin{array}{l}\text { Mudstone and argillaceous phosphate rock } \\
\text { Phosphate rock and mudstone } \\
\text { Phosphate rock } \\
\text { Phosphate rock }\end{array}$ & $\begin{array}{l}1570-\text { RAH } \\
1509-\text { RAH } \\
1508-\text { RAH } \\
1507-\text { RAH }\end{array}$ & $\begin{array}{l}0.7 \\
0.6 \\
1.5 \\
1.8\end{array}$ & $\begin{array}{l}17.1 \\
23.3 \\
33.4 \\
29.5\end{array}$ & $\begin{array}{l}-- \\
-- \\
0.78 \\
2.1\end{array}$ & $\begin{array}{c}-- \\
-- \\
0.44 \\
0.85\end{array}$ & $\begin{array}{r}-- \\
17.54 \\
7.08\end{array}$ & $\begin{array}{r}40.6 \\
28.6 \\
6.1 \\
13.3\end{array}$ & $\begin{array}{l}129.6 \\
130.2 \\
131.7 \\
133.5\end{array}$ & $\begin{array}{l}1,495.85 \\
1,509.83 \\
1,559.93 \\
1,613.03\end{array}$ \\
\hline P- 17 & $\begin{array}{l}\text { Phosphate rock, argillaceous and } \\
\text { mudstone; fos. col. no. 48-JES-95 }\end{array}$ & 1506- RAH & 1.0 & 18.8 & 5.0 & 2.00 & 7.02 & 38.3 & 134.5 & $1,631.83$ \\
\hline $\begin{array}{l}P-16 \\
P-15 \\
P-14\end{array}$ & $\begin{array}{l}\text { Mudstone } \\
\text { Phosphate rock } \\
\text { Phosphate rock }\end{array}$ & $\begin{array}{l}\text { 1505- RAH } \\
1504-\text { RAH } \\
1503-\text { RAH }\end{array}$ & $\begin{array}{l}0.5 \\
1.9 \\
0.5\end{array}$ & $\begin{array}{r}5.3 \\
33.0 \\
35.8\end{array}$ & $\begin{array}{l}9.1 \\
1.4 \\
0.88\end{array}$ & $\begin{array}{l}3.11 \\
0.66 \\
0.32\end{array}$ & $\begin{array}{l}6.74 \\
6.36 \\
4.12\end{array}$ & $\begin{array}{r}69.5 \\
7.6 \\
4.3\end{array}$ & $\begin{array}{l}135.0 \\
136.9 \\
137.4\end{array}$ & $\begin{array}{l}1,634.48 \\
1,697.18 \\
1,715.08\end{array}$ \\
\hline
\end{tabular}




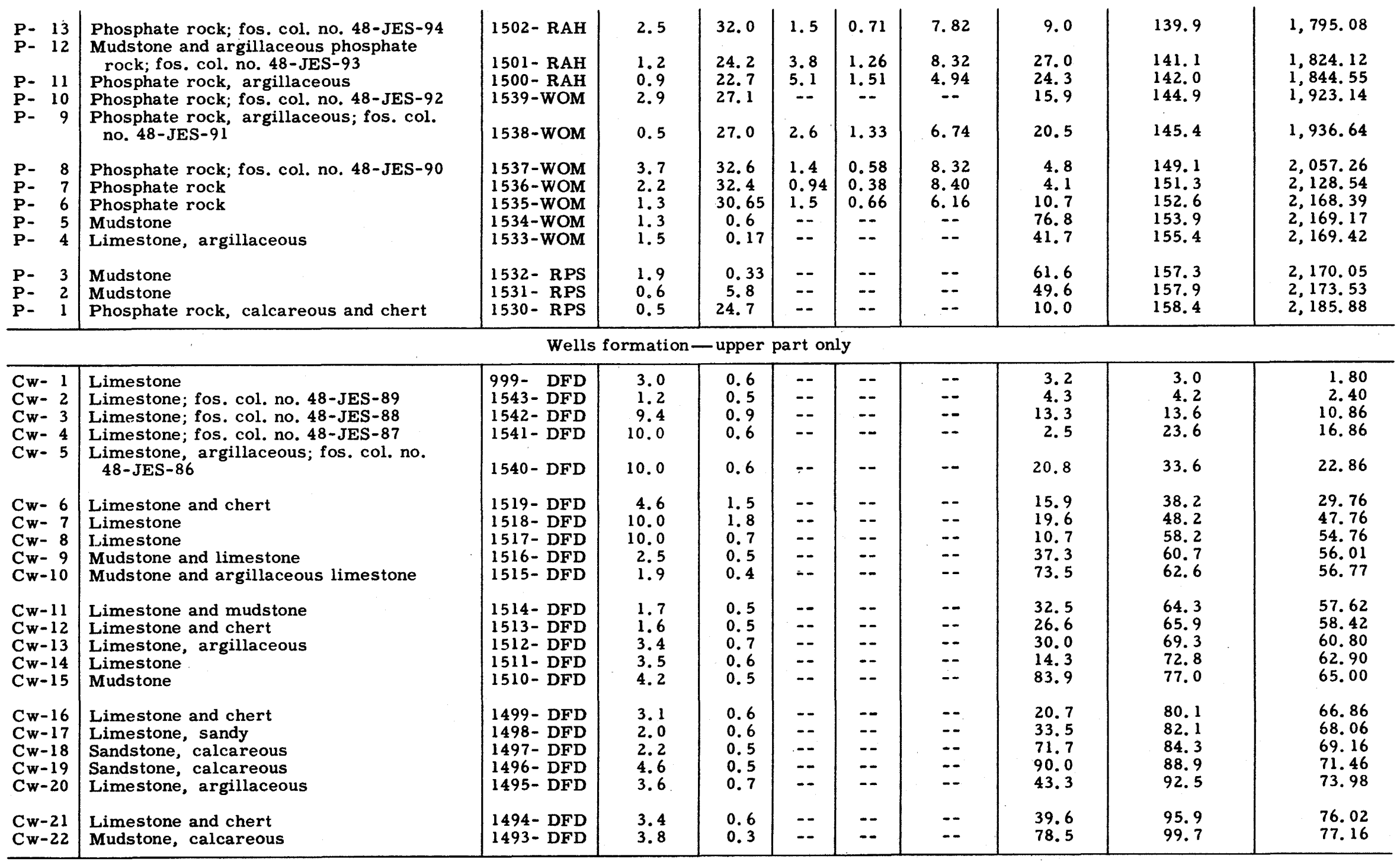


SLUG CREEK VALLEY, IDAHO. LOT NO. 1.278

Part of phosphatic shale member of Phosphoria formation cored in diamond drill hole no. 7 on crest of hill rising above Slug Creek Valley, sec. 30, T. 8 S., R. 44 E., Caribou County, Idaho, on west limb of Schmid syncline. Beds strike north and dip $8^{\circ}$ E. Hole drilled in September and October 1948 by U. S. Bureau of Mines, A. E. Long in charge, and core measured and sampled by D. F. Davidson. Samples analyzed for $\mathrm{P}_{2} \mathrm{O}_{5}$ and acid insoluble by U. S. Bureau of Mines Laboratory, Albany, Oregon.

\begin{tabular}{|c|c|c|c|c|c|c|c|}
\hline \multirow{2}{*}{$\begin{array}{c}\text { Bed } \\
\text { no. }\end{array}$} & \multirow{2}{*}{ Rock description } & \multirow{2}{*}{$\begin{array}{l}\text { Sample } \\
\text { no. }\end{array}$} & \multirow{2}{*}{$\begin{array}{l}\text { Thickness } \\
\text { (feet) }\end{array}$} & \multicolumn{2}{|c|}{$\begin{array}{c}\text { Chemical analyses } \\
\text { (percent) }\end{array}$} & \multirow{2}{*}{$\begin{array}{l}\text { Cumulative } \\
\text { thickness } \\
\text { (feet) }\end{array}$} & \multirow{2}{*}{$\begin{array}{l}\text { Thickness } \mathrm{x} \\
\text { percent } \mathrm{P}_{2} \mathrm{O}_{5} \\
\text { (cumulative) }\end{array}$} \\
\hline & & & & $\mathrm{P}_{2} \mathrm{O}_{5}$ & $\begin{array}{c}\text { Acid } \\
\text { insoluble }\end{array}$ & & \\
\hline \multicolumn{8}{|c|}{ Phosphatic shale member of Phosphoria formation-partial section only } \\
\hline $\begin{array}{l}P-103 \\
P-102 \\
--\end{array}$ & $\begin{array}{l}\text { Mudstone } \\
\text { Core missing } \\
\text { Mudstone } \\
\text { Sample 2723-DFD represents }\end{array}$ & $\begin{array}{l}2722-D F D \\
2723-D F D \\
02\end{array}$ & $\begin{array}{c}0.3 \\
2.8 \\
(0.4)\end{array}$ & $\begin{array}{l}1.1 \\
-- \\
5.6\end{array}$ & $\begin{array}{l}83.7 \\
-- \\
69.3\end{array}$ & $\begin{array}{l}0.3 \\
3.1 \\
--\end{array}$ & $\begin{array}{l}-- \\
--\end{array}$ \\
\hline $\begin{array}{l}P-101 \\
P-100\end{array}$ & $\begin{array}{l}\text { Mudstone } \\
\text { Mudstone }\end{array}$ & $\begin{array}{l}\text { 2724-DFD } \\
2725-\mathrm{DFD}\end{array}$ & $\begin{array}{l}1.1 \\
0.5\end{array}$ & $\begin{array}{l}4.5 \\
4.6\end{array}$ & $\begin{array}{l}70.5 \\
72.8\end{array}$ & $\begin{array}{l}4.2 \\
4.7\end{array}$ & -- \\
\hline $\begin{array}{c}\text { P- } 99 \\
--\end{array}$ & $\begin{array}{l}\text { Core missing } \\
\text { Phosphate rock, argillaceous } \\
\text { Sample } 2726 \text {-DFD represents }\end{array}$ & $2726-\overline{D F D}$ & $\begin{array}{l}3.4 \\
(0.6)\end{array}$ & 28.4 & 22.1 & $\begin{array}{l}8.1 \\
--\end{array}$ & -- \\
\hline P- 9.8 & Phosphate rock & 2727-DFD & 0.4 & 29.3 & 19.5 & 8.5 & -- \\
\hline $\begin{array}{l}\text { P- } 97 \\
\text { P- } 96\end{array}$ & $\begin{array}{l}\text { Core missing } \\
\text { Phosphate rock and mudstone }\end{array}$ & $2728-D F D$ & $\begin{array}{l}0.3 \\
1.9\end{array}$ & 28.7 & 22.3 & $\begin{array}{r}8.8 \\
10.7\end{array}$ & -- \\
\hline $\begin{array}{c}\mathrm{P}-95 \\
--\end{array}$ & $\begin{array}{l}\text { Core missing } \\
\text { Mudstone } \\
\text { Sample } 2729-\text { DFD represents }\end{array}$ & $2729-\mathrm{DFD}$ & $\begin{array}{l}5.1 \\
(0.7)\end{array}$ & 4.7 & $\overline{68.6}$ & $\begin{array}{c}15.8 \\
--\end{array}$ & -- \\
\hline $\begin{array}{l}\text { P- } 94 \\
\text { P- } 93 \\
\text { P- } 92\end{array}$ & $\begin{array}{l}\text { Phosphate rock, argillaceous } \\
\text { Phosphate rock, argillaceous } \\
\text { Core missing }\end{array}$ & $\begin{array}{l}2730-D F D \\
2731-D F D \\
--\end{array}$ & $\begin{array}{l}3.6 \\
0.9 \\
2.6\end{array}$ & $\begin{array}{c}22.7 \\
27.3 \\
--\end{array}$ & $\begin{array}{c}25.6 \\
24.0 \\
--\end{array}$ & $\begin{array}{l}19.4 \\
20.3 \\
22.9\end{array}$ & $\begin{array}{ll}-- \\
--\end{array}$ \\
\hline $\begin{array}{l}\text { P- } 91 \\
\text { P- } 90 \\
\text { P- } 89 \\
\text { P- } 88 \\
\text { P- } 87\end{array}$ & $\begin{array}{l}\text { Phosphate rock, argillaceous } \\
\text { Core missing } \\
\text { Mudstone, phosphatic } \\
\text { Mudstone } \\
\text { Mudstone, phosphatic }\end{array}$ & $\begin{array}{c}2732-D F D \\
-- \\
2733-D F D \\
2734-D F D \\
2735-D F D\end{array}$ & $\begin{array}{l}0.5 \\
1.0 \\
1.1 \\
1.1 \\
2.1\end{array}$ & $\begin{array}{r}24.5 \\
-- \\
19.1 \\
4.5 \\
13.3\end{array}$ & $\begin{array}{l}31.4 \\
-- \\
48.6 \\
81.7 \\
58.2\end{array}$ & $\begin{array}{l}23.4 \\
24.4 \\
25.5 \\
26.6 \\
28.7\end{array}$ & $\begin{array}{l}-- \\
-- \\
-- \\
--\end{array}$ \\
\hline $\begin{array}{l}P-86 \\
P-85 \\
P-84 \\
P-83\end{array}$ & $\begin{array}{l}\text { Mudstone, phosphatic } \\
\text { Core missing } \\
\text { Mudstone, phosphatic } \\
\text { Mudstone, phosphatic }\end{array}$ & $\begin{array}{c}2736-D F D \\
-- \\
2737-D F D \\
2738-D F D\end{array}$ & $\begin{array}{l}1.1 \\
2.5 \\
2.0 \\
2.1\end{array}$ & $\begin{array}{l}11.3 \\
-.- \\
8.2 \\
9.2\end{array}$ & $\begin{array}{c}59.5 \\
-- \\
67.9 \\
63.8\end{array}$ & $\begin{array}{l}29.8 \\
32.3 \\
34.3 \\
36.4\end{array}$ & $\begin{array}{l}-- \\
-- \\
-- \\
--\end{array}$ \\
\hline
\end{tabular}




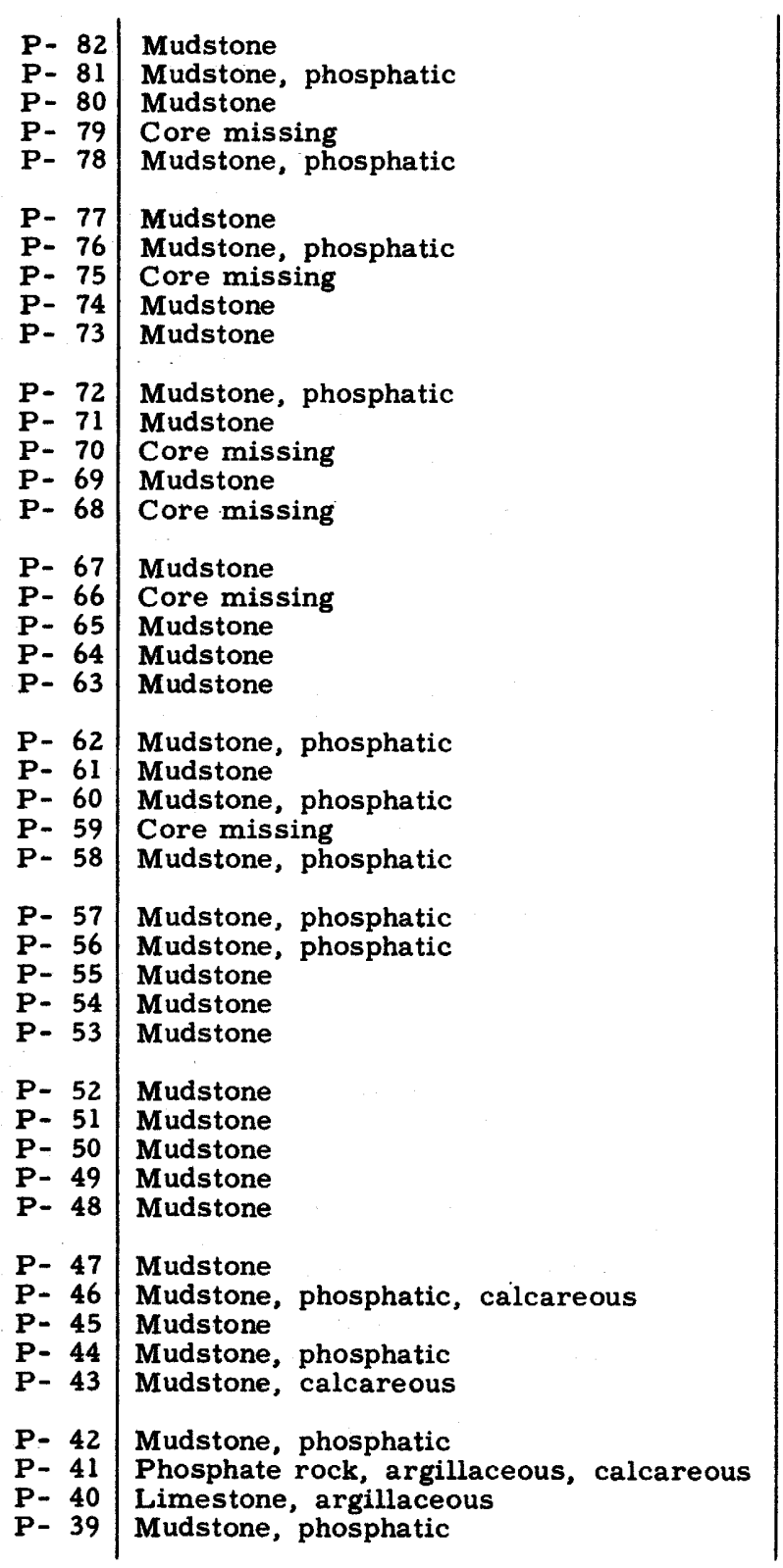

\begin{tabular}{|c|c|c|c|}
\hline $\begin{array}{c}\text { 2739-DFD } \\
2740-\text { DFD } \\
2741 \text {-DFD } \\
-- \\
2742-\text { DFD }\end{array}$ & $\begin{array}{l}1.8 \\
1.6 \\
1.2 \\
1.0 \\
1.7\end{array}$ & $\begin{array}{c}6.1 \\
9.7 \\
5.5 \\
-- \\
10.2\end{array}$ & $\begin{array}{l}70.6 \\
60.5 \\
74.0 \\
-- \\
59.6\end{array}$ \\
\hline $\begin{array}{l}\text { 2743-DFD } \\
\text { 2744-DFD } \\
\text { 2745-DFD } \\
2746 \text {-DFD }\end{array}$ & $\begin{array}{l}1.4 \\
0.5 \\
1.6 \\
0.8 \\
2.6\end{array}$ & $\begin{array}{l}3.0 \\
8.1 \\
-- \\
4.6 \\
0.4\end{array}$ & $\begin{array}{l}77.3 \\
63.5 \\
-- \\
77.6 \\
89.0\end{array}$ \\
\hline $\begin{array}{c}2747-D F D \\
2748-D F D \\
-- \\
2749-D F D \\
--\end{array}$ & $\begin{array}{l}0.9 \\
1.0 \\
2.4 \\
0.8 \\
3.1\end{array}$ & $\begin{array}{l}17.6 \\
1.1 \\
-- \\
0.3 \\
-.\end{array}$ & $\begin{array}{c}48.0 \\
84.5 \\
-- \\
89.1 \\
--\end{array}$ \\
\hline $\begin{array}{c}\text { 2750-DFD } \\
\text { 2751-DFD } \\
2752-\text { DFD } \\
2753 \text {-DFD }\end{array}$ & $\begin{array}{l}2.2 \\
0.6 \\
0.8 \\
1.3 \\
0.6\end{array}$ & $\begin{array}{l}2.2 \\
-. \\
2.8 \\
0.9 \\
6.7\end{array}$ & $\begin{array}{l}83.7 \\
-- \\
82.6 \\
88.5 \\
73.4\end{array}$ \\
\hline $\begin{array}{c}2754-\text { DFD } \\
2755-\text { DFD } \\
2756-\text { DFD } \\
-- \\
2757-\text { DFD }\end{array}$ & $\begin{array}{l}1.6 \\
3.8 \\
0.7 \\
2.4 \\
2.2\end{array}$ & $\begin{array}{r}19.7 \\
4.7 \\
12.3 \\
-- \\
9.9\end{array}$ & $\begin{array}{l}37.0 \\
80.6 \\
49.4 \\
-- \\
55.6\end{array}$ \\
\hline $\begin{array}{l}\text { 2758-DFD } \\
2759-\text { DFD } \\
2760-\text { DFD } \\
2761 \text {-DFD } \\
2762 \text {-DFD }\end{array}$ & $\begin{array}{l}2.3 \\
0.7 \\
1.8 \\
1.7 \\
1.1\end{array}$ & $\begin{array}{r}10.5 \\
13.5 \\
6.4 \\
3.0 \\
0.7\end{array}$ & $\begin{array}{l}47.3 \\
50.7 \\
57.6 \\
79.1 \\
87.6\end{array}$ \\
\hline $\begin{array}{l}\text { 2763-DFD } \\
2764-\text { DFD } \\
2765-\text { DFD } \\
2766-\text { DFD } \\
2767-\text { DFD }\end{array}$ & $\begin{array}{l}0.6 \\
0.3 \\
0.4 \\
0.9 \\
0.6\end{array}$ & $\begin{array}{l}7.5 \\
5.9 \\
3.8 \\
3.9 \\
6.3\end{array}$ & $\begin{array}{l}59.6 \\
69.7 \\
76.5 \\
80.0 \\
71.1\end{array}$ \\
\hline $\begin{array}{l}\text { 2768-DFD } \\
2769-\text { DFD } \\
2770 \text {-DFD } \\
2771 \text {-DFD } \\
2772-D F D\end{array}$ & $\begin{array}{l}2.0 \\
1.9 \\
0.5 \\
0.7 \\
0.5\end{array}$ & $\begin{array}{r}2.3 \\
10.3 \\
3.9 \\
8.4 \\
2.5\end{array}$ & $\begin{array}{l}82.8 \\
49.3 \\
77.8 \\
60.7 \\
49.1\end{array}$ \\
\hline $\begin{array}{l}2773-D F D \\
2774-D F D \\
2775-D F D \\
2776-D F D\end{array}$ & $\begin{array}{l}1.8 \\
1.7 \\
2.9 \\
0.7\end{array}$ & $\begin{array}{r}13.4 \\
14.2 \\
4.1 \\
14.7\end{array}$ & $\begin{array}{l}45.8 \\
35.1 \\
30.2 \\
44.6\end{array}$ \\
\hline
\end{tabular}

\begin{tabular}{|c|c|}
\hline $\begin{array}{l}38.2 \\
39.8 \\
41.0 \\
42.0 \\
43.7\end{array}$ & $\begin{array}{l}-- \\
-- \\
-- \\
--\end{array}$ \\
\hline $\begin{array}{l}45.1 \\
45.6 \\
47.2 \\
48.0 \\
50.6\end{array}$ & $\begin{array}{l}-- \\
-- \\
-- \\
--\end{array}$ \\
\hline $\begin{array}{l}51.5 \\
52.5 \\
54.9 \\
55.7 \\
58.8\end{array}$ & $\begin{array}{l}-- \\
-- \\
-- \\
--\end{array}$ \\
\hline $\begin{array}{l}61.0 \\
61.6 \\
62.4 \\
63.7 \\
64.3\end{array}$ & $\begin{array}{l}-- \\
-- \\
-- \\
--\end{array}$ \\
\hline $\begin{array}{l}65.9 \\
69.7 \\
70.4 \\
72.8 \\
75.0\end{array}$ & $\begin{array}{l}-- \\
-- \\
-- \\
-- \\
21.78\end{array}$ \\
\hline $\begin{array}{l}77.3 \\
78.0 \\
79.8 \\
81.5 \\
82.6\end{array}$ & $\begin{array}{l}45.93 \\
55.38 \\
66.90 \\
72.00 \\
72.77\end{array}$ \\
\hline $\begin{array}{l}83.2 \\
83.5 \\
83.9 \\
84.8 \\
85.4\end{array}$ & $\begin{array}{l}77.27 \\
79.04 \\
80.56 \\
84.07 \\
87.85\end{array}$ \\
\hline $\begin{array}{l}87.4 \\
89.3 \\
89.8 \\
90.5 \\
91.0\end{array}$ & $\begin{array}{r}92.45 \\
112.02 \\
113.97 \\
119.85 \\
121.10\end{array}$ \\
\hline $\begin{array}{l}92.8 \\
94.5 \\
97.4 \\
98.1\end{array}$ & $\begin{array}{l}145.22 \\
169.36 \\
181.25 \\
191.54\end{array}$ \\
\hline
\end{tabular}




\begin{tabular}{|c|c|c|c|c|c|c|c|}
\hline \multirow{2}{*}{$\begin{array}{c}\text { Bed } \\
\text { no. }\end{array}$} & \multirow{2}{*}{ Rock description } & \multirow{2}{*}{$\begin{array}{l}\text { Sample } \\
\text { no. }\end{array}$} & \multirow{2}{*}{$\begin{array}{c}\text { Thickness } \\
\text { (feet) }\end{array}$} & \multicolumn{2}{|c|}{$\begin{array}{c}\text { Chemical analyses } \\
\text { (percent) }\end{array}$} & \multirow{2}{*}{$\begin{array}{c}\text { Cumulative } \\
\text { thickness } \\
\text { (feet) }\end{array}$} & \multirow{2}{*}{$\begin{array}{l}\text { Thickness } x \\
\text { percent } P_{2} \mathrm{O}_{5} \\
\text { (cumulative) }\end{array}$} \\
\hline & & & & $\mathrm{P}_{2} \mathrm{O}_{5}$ & \begin{tabular}{|l|} 
Acid \\
insoluble
\end{tabular} & & \\
\hline $\begin{array}{l}\text { P- } 38 \\
\text { P- } 37 \\
\text { P- 36 } \\
\text { P- } 35 \\
\text { P- } 34\end{array}$ & $\begin{array}{l}\text { Mudstone, phosphatic } \\
\text { Mudstone, phosphatic } \\
\text { Mudstone, calcareous, phosphatic } \\
\text { Limestone } \\
\text { Phosphate rock, argillaceous, calcareous }\end{array}$ & $\begin{array}{l}2777-\text { DFD } \\
2778-\text { DFD } \\
2779-\text { DFD } \\
2780-\text { DFD } \\
2781-\text { DFD }\end{array}$ & $\begin{array}{l}2.0 \\
2.0 \\
1.7 \\
0.8 \\
1.8\end{array}$ & $\begin{array}{l}14.0 \\
13.4 \\
12.7 \\
7.4 \\
21.0\end{array}$ & $\begin{array}{l}47.4 \\
43.8 \\
40.6 \\
18.6 \\
25.1\end{array}$ & $\begin{array}{l}100.1 \\
102.1 \\
103.8 \\
104.6 \\
106.4\end{array}$ & $\begin{array}{l}219.54 \\
246.34 \\
267.93 \\
273.85 \\
311.65\end{array}$ \\
\hline $\begin{array}{l}\text { P- } 33 \\
\text { P- 32 } \\
\text { P- 31 } \\
\text { P- 30 } \\
\text { P- 29 }\end{array}$ & $\begin{array}{l}\text { Core missing } \\
\text { Phosphate rock, calcareous } \\
\text { Limestone and phosphate rock } \\
\text { Limestone } \\
\text { Phosphate rock }\end{array}$ & $\begin{array}{l}-- \\
2782-\text { DFD } \\
2783-\text { DFD } \\
2784-\text { DFD } \\
2785-\text { DFD }\end{array}$ & $\begin{array}{l}1.1 \\
0.8 \\
0.8 \\
1.4 \\
1.6\end{array}$ & $\begin{array}{r}24.6 \\
16.6 \\
6.6 \\
26.8\end{array}$ & $\begin{array}{l}1-. \\
15.4 \\
10.5 \\
13.2 \\
14.7\end{array}$ & $\begin{array}{l}107.5 \\
108.3 \\
109.1 \\
110.5 \\
112.1\end{array}$ & $\begin{array}{l}19.68 \\
32.96 \\
42.20 \\
85.08\end{array}$ \\
\hline $\begin{array}{l}\text { P- } 28 \\
\text { P- } 27 \\
\text { P- 26 } \\
\text { P- } 25 \\
\text { P- } 24\end{array}$ & $\begin{array}{l}\text { Limestone } \\
\text { Mudstone, phosphatic } \\
\text { Mudstone, calcareous, phosphatic } \\
\text { Limestone, argillaceous } \\
\text { Phosphate rock }\end{array}$ & $\begin{array}{l}\text { 2786-DFD } \\
2787 \text {-DFD } \\
2788 \text {-DFD } \\
2789-\text { DFD } \\
2790 \text {-DFD }\end{array}$ & $\begin{array}{l}2.8 \\
1.5 \\
1.7 \\
2.7 \\
1.9\end{array}$ & $\begin{array}{r}4.4 \\
12.8 \\
8.1 \\
0.8 \\
26.9\end{array}$ & $\begin{array}{l}15.1 \\
47.0 \\
45.0 \\
19.8 \\
18.1\end{array}$ & $\begin{array}{l}114.9 \\
116.4 \\
118.1 \\
120.8 \\
122.7\end{array}$ & $\begin{array}{r}97.40 \\
116.60 \\
130.37 \\
132.53 \\
183.64\end{array}$ \\
\hline $\begin{array}{l}\text { P- } 23 \\
\text { P- 22 } \\
\text { P- 21 } \\
\text { P- } 20 \\
\text { P- } 19\end{array}$ & $\begin{array}{l}\text { Phosphate rock } \\
\text { Phosphate rock, argillacaeous } \\
\text { Limestone, argillaceous } \\
\text { Phosphate rock } \\
\text { Phosphate rock, argillaceous }\end{array}$ & $\begin{array}{l}\text { 2791-DFD } \\
2792 \text {-DFD } \\
2793 \text {-DFD } \\
2794-\text { DFD } \\
2795 \text {-DFD }\end{array}$ & $\begin{array}{l}2.2 \\
1.2 \\
1.2 \\
1.1 \\
1.5\end{array}$ & $\begin{array}{l}28.8 \\
18.4 \\
2.4 \\
32.6 \\
27.6\end{array}$ & $\begin{array}{l}18.0 \\
41.6 \\
27.1 \\
9.5 \\
20.3\end{array}$ & $\begin{array}{l}124.9 \\
126.1 \\
127.3 \\
128.4 \\
129.9\end{array}$ & $\begin{array}{l}247.00 \\
269.08 \\
271.96 \\
307.82 \\
349.22\end{array}$ \\
\hline $\begin{array}{l}\text { P- } 18 \\
\text { P- } 17 \\
\text { P- } 16 \\
\text { P- } 15 \\
\text { P- } 14\end{array}$ & $\begin{array}{l}\text { Phosphate rock } \\
\text { Phosphate rock } \\
\text { Phosphate rock } \\
\text { Phoshate rock, calcareous } \\
\text { Phosphate rock, calcareous }\end{array}$ & $\begin{array}{l}\text { 2796-DFD } \\
2797-\text { DFD } \\
2798 \text {-DFD } \\
2799-\text { DFD } \\
2800 \text {-DFD }\end{array}$ & $\begin{array}{l}1.1 \\
0.9 \\
0.7 \\
2.2 \\
1.0\end{array}$ & $\begin{array}{l}32.6 \\
29.8 \\
29.7 \\
16.2 \\
23.0\end{array}$ & $\begin{array}{l}8.6 \\
13.9 \\
15.1 \\
16.9 \\
16.7\end{array}$ & $\begin{array}{l}131.0 \\
131.9 \\
132.6 \\
134.8 \\
135.8\end{array}$ & $\begin{array}{l}385.08 \\
411.90 \\
432.69 \\
468.33 \\
491.33\end{array}$ \\
\hline $\begin{array}{l}\text { P- } 13 \\
\text { P- } 12 \\
\text { P- } 11 \\
\text { P- } 10 \\
\text { P- } 9\end{array}$ & $\begin{array}{l}\text { Phosphate rock, argillaceous } \\
\text { Limestone, phosphatic } \\
\text { Limestone } \\
\text { Phosphate rock } \\
\text { Phosphate rock }\end{array}$ & $\begin{array}{l}\text { 3036-DFD } \\
3020-\text { DFD } \\
3024-D F D \\
2524-D F D \\
2525-D F D\end{array}$ & $\begin{array}{l}1.3 \\
1.0 \\
1.3 \\
0.8 \\
1.8\end{array}$ & $\begin{array}{r}23.5 \\
14.5 \\
8.4 \\
32.0 \\
32.4\end{array}$ & $\begin{array}{r}22.8 \\
12.9 \\
7.1 \\
5.0 \\
3.3\end{array}$ & $\begin{array}{l}137.1 \\
138.1 \\
139.4 \\
140.2 \\
142.0\end{array}$ & $\begin{array}{l}521.88 \\
536.38 \\
547.30 \\
572.90 \\
631.22\end{array}$ \\
\hline $\begin{array}{ll}\text { P- } & 8 \\
\text { P- } & 7 \\
\text { P- } & 5 \\
\text { P- } & 4 \\
\text { P- } & \end{array}$ & $\begin{array}{l}\text { Phosphate rock } \\
\text { Phosphate rock } \\
\text { Phosphate rock } \\
\text { Mudstone } \\
\text { Limestone, argillaceous }\end{array}$ & $\begin{array}{l}\text { 2929-DFD } \\
2930-\text { DFD } \\
3028 \text {-DFD } \\
3029-\text { DFD } \\
3030-\text { DFD }\end{array}$ & $\begin{array}{l}1.7 \\
1.7 \\
1.8 \\
1.1 \\
1.3\end{array}$ & $\begin{array}{l}34.3 \\
34.1 \\
31.5 \\
4.2 \\
0.45\end{array}$ & $\begin{array}{r}2.9 \\
2.6 \\
8.5 \\
70.5 \\
37.9\end{array}$ & $\begin{array}{l}143.7 \\
145.4 \\
147.2 \\
148.3 \\
149.6\end{array}$ & $\begin{array}{l}689.53 \\
747.50 \\
804.20 \\
808.82 \\
809.40\end{array}$ \\
\hline $\begin{array}{ll}\text { P- } & 3 \\
\text { P- } & 2 \\
\text { P- } & 1\end{array}$ & $\begin{array}{l}\text { Mudstone } \\
\text { Phosphate rock } \\
\text { Phosphate rock }\end{array}$ & $\begin{array}{l}\text { 3031-DFD } \\
\text { 3032-DFD } \\
\text { 3033-DFD }\end{array}$ & $\begin{array}{l}1.7 \\
0.3 \\
1.1\end{array}$ & $\begin{array}{r}3.0 \\
31.7 \\
33.6\end{array}$ & $\begin{array}{r}69.9 \\
4.5 \\
2.8\end{array}$ & $\begin{array}{l}151.3 \\
151.6 \\
152.7\end{array}$ & $\begin{array}{l}814.50 \\
824.02 \\
860.98\end{array}$ \\
\hline
\end{tabular}


WEST GEORGETOWN CANYON, IDAHO. LOT NO. 1264.

Phosphatic shale member of Phosphoria formation sampled in trench on west wall of Georgetown Canyon, SE $\frac{1}{4}$ sec. 1 , T. $10 \mathrm{~S}$. , R. $44 \mathrm{E} .$, Bear Lake County, Idaho, on west limb of Georgetown syncline. Beds strike N. $30^{\circ} \mathrm{E}$. and dip $50^{\circ} \mathrm{E}$. Section measured by D. F. Davidson and F. W.

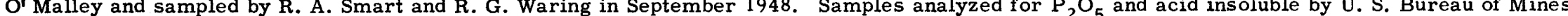

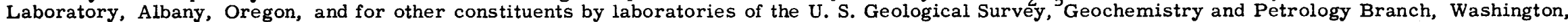
D. C.

\begin{tabular}{|c|c|c|c|c|c|c|c|c|c|c|}
\hline \multirow[b]{2}{*}{$\begin{array}{l}\text { Bed } \\
\text { no. }\end{array}$} & \multirow[b]{2}{*}{ Rock description } & \multirow[b]{2}{*}{$\begin{array}{c}\text { Sample } \\
\text { no. }\end{array}$} & \multirow[b]{2}{*}{$\begin{array}{c}\text { Thickness } \\
\text { (feet) }\end{array}$} & \multicolumn{5}{|c|}{ Chemical analyses (percent) } & \multirow{2}{*}{$\begin{array}{c}\text { Cumulative } \\
\text { thickness } \\
\text { (feet) }\end{array}$} & \multirow{2}{*}{$\begin{array}{l}\text { Thickness } x \\
\text { percent } \mathrm{P}_{2} \mathrm{O}_{5} \\
\text { (cumulative) }\end{array}$} \\
\hline & & & & $\mathrm{P}_{2} \mathrm{O}_{5}$ & $\mathrm{Al}_{2} \mathrm{O}_{3}$ & $\mathrm{Fe}_{2} \mathrm{O}_{3}$ & $\begin{array}{l}\text { Loss on } \\
\text { ignition }\end{array}$ & $\begin{array}{c}\text { Acid } \\
\text { insoluble }\end{array}$ & & \\
\hline
\end{tabular}

Rex member of Phosphoria formation_basal beds only

\begin{tabular}{|c|c|c|c|c|c|c|c|c|c|c|}
\hline $\begin{array}{l}R-7 \\
R-6 \\
R-5 \\
R-4 \\
R-3\end{array}$ & $\begin{array}{l}\text { Chert } \\
\text { Chert } \\
\text { Mudstone } \\
\text { Mudstone } \\
\text { Chert }\end{array}$ & $\begin{array}{l}2640-\text { WOM } \\
2639-\text { WOM } \\
2638-\text { WOM } \\
2637-\text { WOM } \\
2636-\text { WOM }\end{array}$ & $\begin{array}{l}9.0 \\
1.2 \\
0.6 \\
0.8 \\
3.9\end{array}$ & $\begin{array}{l}0.5 \\
0.9 \\
2.1 \\
2.2 \\
1.1\end{array}$ & $\begin{array}{l}-- \\
-- \\
-- \\
--\end{array}$ & $\begin{array}{l}-- \\
-- \\
-- \\
--\end{array}$ & $\begin{array}{l}-- \\
-- \\
-- \\
--\end{array}$ & $\begin{array}{l}92.5 \\
91.9 \\
72.5 \\
80.8 \\
91.9\end{array}$ & $\begin{array}{r}9.0 \\
10.2 \\
10.8 \\
11.6 \\
15.5\end{array}$ & $\begin{array}{r}4.50 \\
5.58 \\
6.84 \\
8.60 \\
12.89\end{array}$ \\
\hline $\begin{array}{l}R-2 \\
R-1\end{array}$ & $\begin{array}{l}\text { Chert } \\
\text { Chert }\end{array}$ & $\begin{array}{l}2635-W O M \\
2634-W O M\end{array}$ & $\begin{array}{l}3.3 \\
5.0\end{array}$ & $\begin{array}{l}0.9 \\
0.9\end{array}$ & $=$ & -- & -- & $\begin{array}{l}91.2 \\
92.1\end{array}$ & $\begin{array}{l}18.8 \\
23.8\end{array}$ & $\begin{array}{l}15.86 \\
20.36\end{array}$ \\
\hline
\end{tabular}

Phosphatic shale member of Phosphoria formation

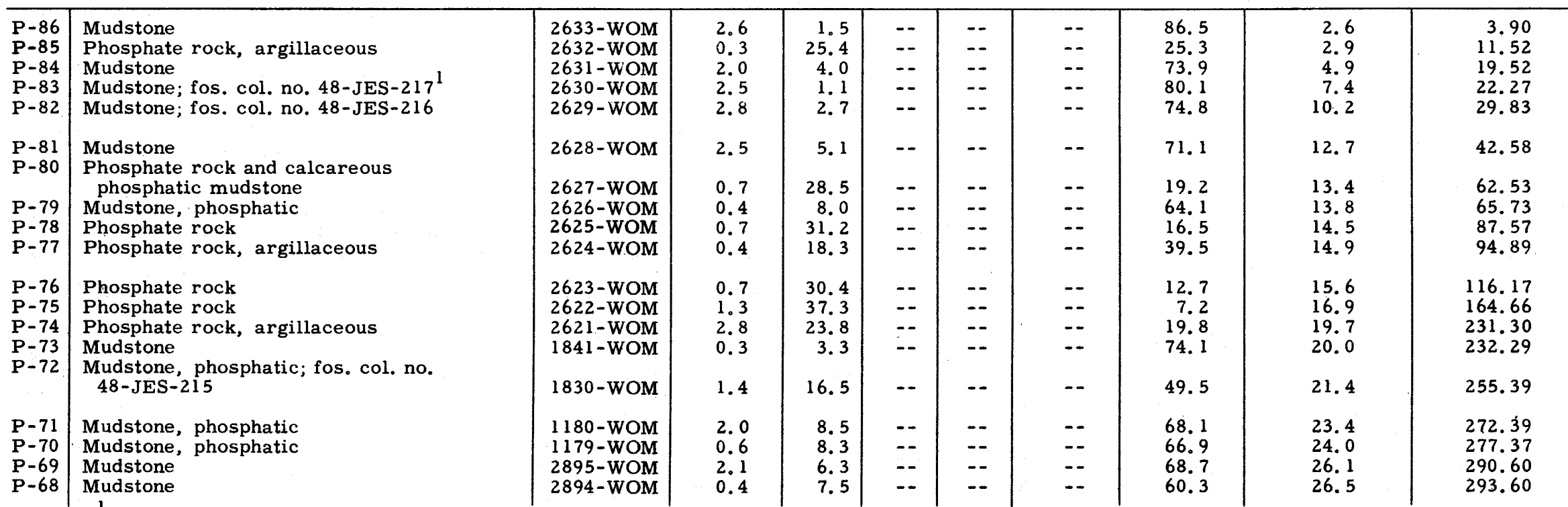

\footnotetext{
${ }^{1}$ Fossil collection made by J. E. Smedley, Paleontology and Stratigraphy Branch, U. S. Geological Survey.
} 


\begin{tabular}{|c|c|c|c|c|c|c|c|c|c|c|}
\hline \multirow[b]{2}{*}{$\begin{array}{c}\text { Bed } \\
\text { no. }\end{array}$} & \multirow[b]{2}{*}{ Rock description } & \multirow[b]{2}{*}{$\begin{array}{l}\text { Sample } \\
\text { no. }\end{array}$} & \multirow{2}{*}{$\underset{\text { (feet) }}{\text { Thickness }}$} & \multicolumn{5}{|c|}{ Chemical analyses (percent) } & \multirow{2}{*}{$\begin{array}{l}\text { Cumulative } \\
\text { thickness } \\
(\text { feet })\end{array}$} & \multirow{2}{*}{$\begin{array}{l}\text { Thickness } \mathrm{x} \\
\text { percent } \mathrm{P}_{2} \mathrm{O}_{5} \\
\text { (cumulative) }\end{array}$} \\
\hline & & & & $\mathrm{P}_{2} \mathrm{O}_{5}$ & $\mathrm{Al}_{2} \mathrm{O}_{3}$ & $\mathrm{Fe}_{2} \mathrm{O}_{3}$ & $\begin{array}{l}\text { Loss on } \\
\text { ignition }\end{array}$ & $\begin{array}{l}\text { Acid } \\
\text { insoluble }\end{array}$ & & \\
\hline $\begin{array}{l}P-67 \\
P-66 \\
P-65 \\
P-64 \\
P-63\end{array}$ & $\begin{array}{l}\text { Mudstone } \\
\text { Mudstone } \\
\text { Mudstone } \\
\text { Mudstone } \\
\text { Mudstone, phosphatic, calcareous }\end{array}$ & $\begin{array}{l}1178-\text { WOM } \\
2649-\text { WOM } \\
2648-\text { WOM } \\
2647-\text { WOM } \\
2646-\text { WOM }\end{array}$ & $\begin{array}{l}1.1 \\
0.7 \\
0.5 \\
2.0 \\
1.8\end{array}$ & $\begin{array}{r}4.8 \\
4.8 \\
0.9 \\
2.5 \\
14.3\end{array}$ & $\begin{array}{l}-- \\
-- \\
-- \\
--\end{array}$ & $\begin{array}{l}-- \\
-- \\
-- \\
--\end{array}$ & $\begin{array}{l}-- \\
-- \\
-- \\
--\end{array}$ & $\begin{array}{l}63.2 \\
74.5 \\
86.1 \\
80.4 \\
41.5\end{array}$ & $\begin{array}{l}27.6 \\
28.3 \\
28.8 \\
30.8 \\
32.6\end{array}$ & $\begin{array}{l}298.88 \\
302.24 \\
302.69 \\
307.69 \\
333.43\end{array}$ \\
\hline $\begin{array}{l}P-62 \\
P-61 \\
P-60 \\
P-59 \\
P-58\end{array}$ & $\begin{array}{l}\text { Mudstone } \\
\text { Mudstone } \\
\text { Mudstone and argillaceous phosphate rock } \\
\text { Phosphate rock; fos. col. no. } 48-\mathrm{JES}-214 \\
\text { Mudstone, phosphatic; fos. col. no. } \\
\quad 48 \text {-JES-213 }\end{array}$ & $\begin{array}{l}2645-\text { WOM } \\
2644-\text { WOM } \\
2643-\text { WOM } \\
2642-\text { WOM } \\
2641-\text { WOM }\end{array}$ & $\begin{array}{l}0.8 \\
1.9 \\
2.0 \\
0.5 \\
1.1\end{array}$ & $\begin{array}{r}1.4 \\
3.2 \\
2.6 \\
30.7 \\
8.3\end{array}$ & $\begin{array}{l}-- \\
-- \\
-- \\
--\end{array}$ & $\begin{array}{l}-- \\
-- \\
-- \\
--\end{array}$ & $\begin{array}{l}-- \\
-- \\
-- \\
--\end{array}$ & $\begin{array}{l}87.6 \\
82.9 \\
83.5 \\
14.9 \\
68.5\end{array}$ & $\begin{array}{l}33.4 \\
35.3 \\
37.3 \\
37.8 \\
38.9\end{array}$ & $\begin{array}{l}334.55 \\
340.63 \\
345.83 \\
361.18 \\
370.31\end{array}$ \\
\hline $\begin{array}{l}P-57 \\
P-56 \\
P-55 \\
P-54 \\
P-53\end{array}$ & $\begin{array}{l}\text { Phosphate rock; fos. col. no. } 48-J E S-212 \\
\text { Mudstone, phosphatic } \\
\text { Phosphate rock } \\
\text { Mudstone, phosphatic } \\
\text { Mudstone, phosphatic, calcareous }\end{array}$ & $\begin{array}{l}2900-\text { WOM } \\
2893-\text { WOM } \\
2899-\text { WOM } \\
2898-\text { WOM } \\
2897-\text { WOM }\end{array}$ & $\begin{array}{l}0.6 \\
0.3 \\
0.9 \\
0.6 \\
0.5\end{array}$ & $\begin{array}{r}33.8 \\
15.3 \\
31.0 \\
9.3 \\
15.1\end{array}$ & $\begin{array}{l}-- \\
-- \\
-- \\
--\end{array}$ & $\begin{array}{l}-- \\
-- \\
-- \\
--\end{array}$ & $\begin{array}{l}-- \\
-- \\
-- \\
--\end{array}$ & $\begin{array}{l}8.2 \\
45.5 \\
11.4 \\
62.2 \\
41.3\end{array}$ & $\begin{array}{l}39.5 \\
39.8 \\
40.7 \\
41.3 \\
41.8\end{array}$ & $\begin{array}{l}390.59 \\
395.18 \\
423.08 \\
428.66 \\
436.21\end{array}$ \\
\hline $\begin{array}{l}P-52 \\
P-51 \\
P-50\end{array}$ & $\begin{array}{l}\text { Mudstone } \\
\text { Phosphate rock, argillaceous } \\
\text { Mudstone }\end{array}$ & $\begin{array}{l}2896-\text { WOM } \\
2892-\text { WOM } \\
2891-\text { WOM }\end{array}$ & $\begin{array}{l}1.3 \\
0.3 \\
1.8\end{array}$ & $\begin{array}{r}3.6 \\
19.4 \\
2.1\end{array}$ & -- & $\begin{array}{l}-- \\
-- \\
--\end{array}$ & $\begin{array}{l}-- \\
-- \\
--\end{array}$ & $\begin{array}{l}78.9 \\
33.3 \\
81.2\end{array}$ & $\begin{array}{l}43.1 \\
43.4 \\
45.2\end{array}$ & $\begin{array}{l}440.89 \\
446.71 \\
450.49\end{array}$ \\
\hline & Beds $P-49$ and $P-50$ are separated by a fa & & & & & & & & & \\
\hline $\begin{array}{l}P-49 \\
P-48\end{array}$ & $\begin{array}{l}\text { Phosphate rock, calcareous, argillaceous } \\
\text { Mudstone }\end{array}$ & $\begin{array}{l}2888-W O M \\
2887-\text { WOM }\end{array}$ & $\begin{array}{l}1.1 \\
1.8\end{array}$ & $\begin{array}{r}16.1 \\
5.4\end{array}$ & -- & -- & -- & $\begin{array}{l}29.1 \\
70.3\end{array}$ & $\begin{array}{l}46.3 \\
48.1\end{array}$ & $\begin{array}{l}468.20 \\
477.92\end{array}$ \\
\hline$P-47$ & $\begin{array}{l}\text { Mudstone, phosphatic, calcareous; } \\
\text { fos. col. no. } 48-J E S-211\end{array}$ & 2886-WOM & 2.6 & 13.4 & -- & -- & -- & 43.1 & 50.7 & 512.76 \\
\hline$P-46$ & $\begin{array}{l}\text { Mudstone, phosphatic; fos. col. no. } \\
48 \text {-JES- } 210 \\
\text { Limestone concretion, argillaceous }\end{array}$ & $\begin{array}{l}2885-W O M \\
2884-W O M\end{array}$ & $\begin{array}{c}1.2 \\
(2.4)\end{array}$ & $\begin{array}{l}8.2 \\
0.6\end{array}$ & -- & -- & -- & $\begin{array}{l}61.1 \\
19.5\end{array}$ & 51.9 & $\begin{array}{c}522.60 \\
--\end{array}$ \\
\hline $\begin{array}{l}P-45 \\
P-44 \\
P-43 \\
P-42 \\
P-41\end{array}$ & $\begin{array}{l}\text { Chert } \\
\text { Mudstone } \\
\text { Mudstone; fos. col. no. } 48 \text {-JES-209 } \\
\text { Mudstone; fos. col. no. } 48 \text {-JES-208 } \\
\text { Mudstone }\end{array}$ & $\begin{array}{l}2883-\text { WOM } \\
2882-\text { WOM } \\
2881-\text { WOM } \\
2880-\text { WOM } \\
2879-\text { WOM }\end{array}$ & $\begin{array}{l}0.3 \\
3.6 \\
2.0 \\
3.4 \\
1.6\end{array}$ & $\begin{array}{l}0.6 \\
2.7 \\
4.1 \\
3.9 \\
2.2\end{array}$ & $\begin{array}{l}-- \\
-- \\
-- \\
--\end{array}$ & $\begin{array}{l}-- \\
-- \\
-- \\
--\end{array}$ & $\begin{array}{l}-- \\
-- \\
-- \\
--\end{array}$ & $\begin{array}{l}90.0 \\
78.3 \\
71.3 \\
76.7 \\
83.5\end{array}$ & $\begin{array}{l}52.2 \\
55.8 \\
57.8 \\
61.2 \\
62.8\end{array}$ & $\begin{array}{l}522.78 \\
532.50 \\
540.70 \\
553.96 \\
557.48\end{array}$ \\
\hline $\begin{array}{l}P-35 \\
P-34\end{array}$ & $\begin{array}{l}\text { Mudstone, phosphatic } \\
\text { Phosphate rock and phosphatic mudstone }\end{array}$ & $\begin{array}{l}\text { 2928- DFD } \\
2927-\text { DFD }\end{array}$ & $\begin{array}{l}0.6 \\
0.8\end{array}$ & $\begin{array}{l}15.8 \\
15.4\end{array}$ & -- & -- & -- & $\begin{array}{l}41.8 \\
40.6\end{array}$ & $\begin{array}{l}68.0 \\
68.8\end{array}$ & $\begin{array}{l}619.46 \\
631.78\end{array}$ \\
\hline
\end{tabular}


P-33 Mudstone, phosphatic

-32 Phosphate rock, argillaceous

P-31 Phosphate rock, argillaceous

P-30 Phosphate rock

P-29 Mudstone, calcareous

P-28 Phosphate rock and phosphatic mudstone

$\mathrm{P}-27$

$\mathrm{P}-27$

$\mathrm{P}-26$ Mudstone, phosphatic

Limestone

P-24 Mudstone, phosphatic

Mudstone, phosphatic

-23 Limestone, argillaceous

$\mathrm{P}-22$ Phosphate rock

$P-21$ Phosphate rock

P-20 Phosphate rock, argillaceous

Limestone concretion

P-19 Phosphate rock

P-18 Phosphate rock, argillaceous

P-17 Mudstone, calcareous, phosphatic

P-16 Mudstone, phosphatic

-- Limestone concretion; fos. col. no. 48-JES- 207

P-15 Mudstone, phosphatic

P-14 Phosphate rock

P-13 Phosphate rock, argillaceous

$\mathrm{P}-12$ Phosphate rock, argillaceous

P-11 Phosphate rock, argillaceous

P-10 Mudstone, phosphatic

P- 9 Mudstone, phosphatic

P - 8 Phosphate rock, argillaceous

P- 7 Phosphate rock

P- 6 Mudstone, phosphatic

P- 5 Phosphate rock, argillaceous

P- 4 Phosphate rock

P- 3 Phosphate rock, argillaceous, calcareous

P- 2 Limestone; fos. col. no. 48-JES-206

P- 1 Phosphate rock

\begin{tabular}{|c|c|c|c|c|c|c|c|c|}
\hline $\begin{array}{l}2926-\text { DFD } \\
2925-\text { DFD } \\
2924-\text { DFD } \\
2923-\text { DFD } \\
2922-\text { DFD }\end{array}$ & $\begin{array}{l}0.5 \\
0.7 \\
0.5 \\
0.6 \\
0.35\end{array}$ & $\begin{array}{r}11.8 \\
18.3 \\
17.5 \\
23.9 \\
3.8\end{array}$ & $\begin{array}{l}-- \\
-- \\
-- \\
--\end{array}$ & $\begin{array}{l}-- \\
-- \\
-- \\
--\end{array}$ & $\begin{array}{l}-- \\
-- \\
-- \\
--\end{array}$ & $\begin{array}{l}51.0 \\
34.4 \\
40.7 \\
15.4 \\
43.6\end{array}$ & $\begin{array}{l}69.3 \\
70.0 \\
70.5 \\
71.1 \\
71.45\end{array}$ & $\begin{array}{l}637.68 \\
650.49 \\
659.24 \\
673.58 \\
674.91\end{array}$ \\
\hline $\begin{array}{l}2870-\text { DFD } \\
2869-\text { DFD } \\
2868-\text { DFD } \\
2867-\text { DFD } \\
2866-\text { DFD }\end{array}$ & $\begin{array}{l}1.5 \\
0.70 \\
1.3 \\
1.0 \\
2.3\end{array}$ & $\begin{array}{r}23.0 \\
10.7 \\
2.8 \\
10.6 \\
9.6\end{array}$ & $\begin{array}{l}-- \\
-- \\
-- \\
--\end{array}$ & $\begin{array}{l}-- \\
-- \\
-- \\
--\end{array}$ & $\begin{array}{l}-- \\
-- \\
-- \\
--\end{array}$ & $\begin{array}{l}23.6 \\
56.1 \\
17.8 \\
55.5 \\
58.2\end{array}$ & $\begin{array}{l}72.95 \\
73.65 \\
74.95 \\
75.95 \\
78.25\end{array}$ & $\begin{array}{l}709.41 \\
716.90 \\
720.54 \\
731.14 \\
753.22\end{array}$ \\
\hline $\begin{array}{l}2865-\text { DFD } \\
2864-\text { DFD } \\
2863-\text { DFD } \\
2862-\text { DFD } \\
2861-\text { DFD }\end{array}$ & $\begin{array}{c}1.1 \\
0.9 \\
0.85 \\
0.75 \\
(1.0-1.2)\end{array}$ & $\begin{array}{r}0.9 \\
26.5 \\
26.3 \\
22.8 \\
4.2\end{array}$ & $\begin{array}{l}-- \\
-- \\
-- \\
--\end{array}$ & $\begin{array}{l}-- \\
-- \\
-- \\
--\end{array}$ & $\begin{array}{l}-- \\
-- \\
-- \\
--\end{array}$ & $\begin{array}{r}23.5 \\
19.9 \\
18.3 \\
29.4 \\
5.5\end{array}$ & $\begin{array}{l}79.35 \\
80.25 \\
81.10 \\
81.85 \\
--\end{array}$ & $\begin{array}{c}754.21 \\
778.06 \\
800.42 \\
817.52 \\
\ldots-\end{array}$ \\
\hline $\begin{array}{l}2910-\text { DFD } \\
2909-\text { DFD } \\
2908-\text { DFD } \\
2907-\text { DFD }\end{array}$ & $\begin{array}{l}2.0 \\
2.1 \\
0.8 \\
0.7\end{array}$ & $\begin{array}{r}27.8 \\
24.1 \\
8.3 \\
14.5\end{array}$ & $\begin{array}{l}-- \\
-- \\
--\end{array}$ & $\begin{array}{l}-- \\
-- \\
--\end{array}$ & $\begin{array}{l}-- \\
-- \\
--\end{array}$ & $\begin{array}{l}13.4 \\
23.5 \\
49.4 \\
47.7\end{array}$ & $\begin{array}{l}83.85 \\
85.95 \\
86.75 \\
87.45\end{array}$ & $\begin{array}{l}873.12 \\
923.73 \\
930.37 \\
940.52\end{array}$ \\
\hline 2906- DFD & $(0.9)$ & 4.1 & -- & -- & -- & 19.5 & -- & -- \\
\hline $\begin{array}{l}2905-\text { DFD } \\
2904-\text { DFD } \\
2903-\text { DFD } \\
2902-\text { DFD } \\
2901-\text { DFD }\end{array}$ & $\begin{array}{l}1.6 \\
1.8 \\
1.6 \\
1.3 \\
1.0\end{array}$ & $\begin{array}{l}13.7 \\
27.4 \\
26.4 \\
24.4 \\
25.7\end{array}$ & $\begin{array}{l}-- \\
3.0 \\
2.5 \\
3.6 \\
3.3\end{array}$ & $\begin{array}{l}-. \\
1.2 \\
0.89 \\
2.5 \\
1.2\end{array}$ & $\begin{array}{l}-. \\
9.16 \\
7.96 \\
9.62 \\
8.18\end{array}$ & $\begin{array}{l}50.2 \\
17.7 \\
22.3 \\
23.9 \\
23.7\end{array}$ & $\begin{array}{l}89.05 \\
90.85 \\
92.45 \\
93.75 \\
94.75\end{array}$ & $\begin{array}{r}962.44 \\
1,011.76 \\
1,054.00 \\
1,085.72 \\
1,111.42\end{array}$ \\
\hline $\begin{array}{l}2920 \text { - DFD } \\
2919 \text { - DFD } \\
2918 \text { - DFD } \\
2917 \text { - DFD } \\
2916 \text { - DFD }\end{array}$ & $\begin{array}{l}0.6 \\
1.0 \\
2.2 \\
4.0 \\
1.4\end{array}$ & $\begin{array}{l}12.5 \\
13.7 \\
26.5 \\
27.2 \\
16.8\end{array}$ & $\begin{array}{l}8.3 \\
8.2 \\
3.7 \\
1.2 \\
6.6\end{array}$ & $\begin{array}{l}2.5 \\
2.4 \\
1.3 \\
0.86 \\
2.0\end{array}$ & $\begin{array}{l}8.32 \\
7.06 \\
7.74 \\
6.60 \\
7.02\end{array}$ & $\begin{array}{l}55.1 \\
52.8 \\
21.4 \\
19.2 \\
44.1\end{array}$ & $\begin{array}{r}95.35 \\
96.35 \\
98.55 \\
102.55^{\circ} \\
103.95\end{array}$ & $\begin{array}{l}1,118.92 \\
1,132.62 \\
1,190.92 \\
1,299.72 \\
1,323.24\end{array}$ \\
\hline $\begin{array}{l}2915-\text { DFD } \\
2914-\text { DFD } \\
2913-\text { DFD } \\
2912-\text { DFD } \\
2911-\text { DFD }\end{array}$ & $\begin{array}{l}1.0 \\
1.4 \\
0.7 \\
0.5 \\
0.22\end{array}$ & $\begin{array}{r}21.5 \\
32.6 \\
13.4 \\
6.5 \\
34.7\end{array}$ & $\begin{array}{l}5.0 \\
1.2 \\
5.9 \\
2.7 \\
1.5\end{array}$ & $\begin{array}{l}1.4 \\
0.60 \\
2.2 \\
1.1 \\
0.90\end{array}$ & $\begin{array}{r}5.82 \\
6.26 \\
13.10 \\
35.30 \\
3.02\end{array}$ & $\begin{array}{r}32.6 \\
6.1 \\
33.6 \\
10.3 \\
5.6\end{array}$ & $\begin{array}{l}104.95 \\
106.35 \\
107.05 \\
107.55 \\
107.77\end{array}$ & $\begin{array}{l}1,344.74 \\
1,390.38 \\
1,399.76 \\
1,403.01 \\
1,410.64\end{array}$ \\
\hline
\end{tabular}

Wells formation

\begin{tabular}{|c|c|c|c|c|c|c|c|c|c|c|}
\hline $\mathrm{Cw}-1$ & Limestone & $2921-D F D$ & 0.5 & 3.3 & -- & -- & -- & 5.0 & 0.5 & 1.65 \\
\hline
\end{tabular}


EAST GEORGETOWN CANYON, IDAHO. LOT NO. 1267.

Phosphatic shale member of Phosphoria formation sampled in bulldozer trench of Central Farmers' Fertilizer Cooperative, on east side of

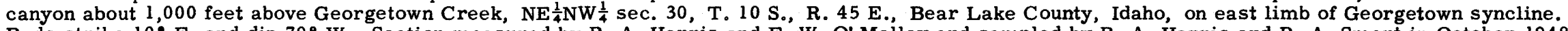

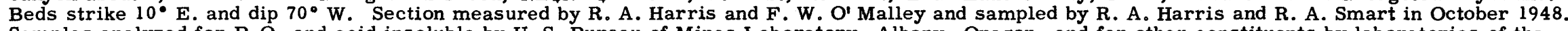

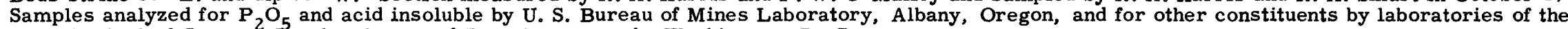
U. S. Geological Survey, Geochemistry and Petrology Branch, Washington, D. C.

\begin{tabular}{|c|c|c|c|c|c|c|c|c|c|c|}
\hline \multirow{2}{*}{$\begin{array}{c}\text { Bed } \\
\text { no. }\end{array}$} & \multirow{2}{*}{ Rock description } & \multirow{2}{*}{$\begin{array}{c}\text { Sample } \\
\text { no. }\end{array}$} & \multirow{2}{*}{$\begin{array}{c}\text { Thickness } \\
\text { (feet) }\end{array}$} & \multicolumn{5}{|c|}{ Chemical analyses (percent) } & \multirow{2}{*}{$\begin{array}{c}\text { Cumulative } \\
\text { thickness } \\
\text { (feet) }\end{array}$} & \multirow{2}{*}{$\begin{array}{l}\text { Thickness } \mathrm{x} \\
\text { percent } \mathrm{P}_{2} \mathrm{O}_{5} \\
\text { (cumulative) }\end{array}$} \\
\hline & & & & $\mathrm{P}_{2} \mathrm{O}_{5}$ & $\mathrm{Al}_{2} \mathrm{O}_{3}$ & $\mathrm{Fe}_{2} \mathrm{O}_{3}$ & $\begin{array}{l}\text { Loss on } \\
\text { ignition }\end{array}$ & $\begin{array}{c}\text { Acid } \\
\text { insoluble }\end{array}$ & & \\
\hline
\end{tabular}

Rex member of Phosphoria formation-basal beds only

\begin{tabular}{|c|c|c|c|c|c|c|c|c|c|c|}
\hline $\begin{array}{ll}R- & 3 \\
R- & 2 \\
R- & 1\end{array}$ & $\begin{array}{l}\text { Chert } \\
\text { Chert } \\
\text { Mudstone, calcareous }\end{array}$ & $\begin{array}{l}3240-\text { WOM } \\
3260-\text { WOM } \\
3259-\text { WOM }\end{array}$ & $\begin{array}{l}3.0 \\
3.5 \\
1.0\end{array}$ & $\begin{array}{l}0.8 \\
1.1 \\
1.5\end{array}$ & $\begin{array}{l}-- \\
-- \\
--\end{array}$ & $\begin{array}{l}-- \\
-- \\
--\end{array}$ & -- & $\begin{array}{l}91.3 \\
90.6 \\
69.8\end{array}$ & $\begin{array}{l}3.0 \\
6.5 \\
7.5\end{array}$ & $\begin{array}{l}2.40 \\
6.25 \\
7.75\end{array}$ \\
\hline
\end{tabular}

Phosphatic shale member of Phosphoria formation-base not exposed

\begin{tabular}{l} 
Phosphatic shale member of Phosphoria formation-base not exposed \\
\hline
\end{tabular}

\begin{tabular}{|c|c|}
\hline $\begin{array}{l}P-108 \\
P-107 \\
P-106 \\
P-105 \\
P-104\end{array}$ & $\begin{array}{l}\text { Mudstone } \\
\text { Mudstone } \\
\text { Phosphate rock } \\
\text { Mudstone } \\
\text { Mudstone, phosphatic }\end{array}$ \\
\hline $\begin{array}{l}P-103 \\
P-102 \\
P-101 \\
P-100 \\
P-99\end{array}$ & $\begin{array}{l}\text { Mudstone } \\
\text { Mudstone, calcareous } \\
\text { Mudstone } \\
\text { Mudstone; fos. col. no. } 48-J E S-370^{1} \\
\text { Mudstone }\end{array}$ \\
\hline $\begin{array}{l}\text { P- } 98 \\
\text { P- } 97\end{array}$ & $\begin{array}{l}\text { Mudstone; fos. col. no. 48-JES-369 } \\
\text { Mudstone, calcareous; fos. col. no. } \\
48-J E S-368\end{array}$ \\
\hline $\begin{array}{l}P-96 \\
P-95 \\
P-94\end{array}$ & $\begin{array}{l}\text { Phosphate rock, argillaceous } \\
\text { Mudstone } \\
\text { Mudstone, phosphatic }\end{array}$ \\
\hline $\begin{array}{l}P-93 \\
P=92 \\
P=91 \\
P-90 \\
P-89\end{array}$ & $\begin{array}{l}\text { Mudstone } \\
\text { Mudstone, phosphatic } \\
\text { Mudstone, phosphatic } \\
\text { Phosphate rock } \\
\text { Mudstone, phosphatic }\end{array}$ \\
\hline $\begin{array}{l}P-88 \\
P=87 \\
P-86 \\
P=85 \\
P-84\end{array}$ & $\begin{array}{l}\text { Phosphate rock } \\
\text { Mudstone } \\
\text { Phosphate rock and phosphatic mudstone } \\
\text { Mudstone and phosphate rock } \\
\text { Phosphate rock }\end{array}$ \\
\hline
\end{tabular}

\begin{tabular}{|c|c|c|c|c|}
\hline $\begin{array}{l}3258-W O M \\
3257-W O M \\
3256-W O M \\
3255-W O M \\
3254-W O M\end{array}$ & $\begin{array}{l}0.5 \\
0.6 \\
0.3 \\
1.0 \\
1.0\end{array}$ & $\begin{array}{r}3.6 \\
3.0 \\
26.1 \\
3.3 \\
8.4\end{array}$ & $\begin{array}{l}-- \\
-- \\
-- \\
--\end{array}$ & $\begin{array}{l}-- \\
-- \\
-- \\
--\end{array}$ \\
\hline $\begin{array}{l}3253-\text { WOM } \\
3252-\text { WOM } \\
3251-\text { WOM } \\
3250-\text { WOM } \\
3249-\text { WOM }\end{array}$ & $\begin{array}{l}3.3 \\
3.0 \\
1.5 \\
2.0 \\
0.3\end{array}$ & $\begin{array}{l}1.0 \\
0.8 \\
2.9 \\
2.4 \\
6.1\end{array}$ & $\begin{array}{l}-- \\
-- \\
-- \\
--\end{array}$ & $\begin{array}{l}-- \\
-- \\
-- \\
--\end{array}$ \\
\hline 3248-WOM & 2.5 & 2. 7 & - & -- \\
\hline $\begin{array}{l}3247-W O M \\
3246-W O M \\
3245-W O M \\
3244-W O M\end{array}$ & $\begin{array}{l}2.5 \\
0.3 \\
0.9 \\
0.3\end{array}$ & $\begin{array}{r}3.7 \\
24.0 \\
4.5 \\
11.5\end{array}$ & $\begin{array}{l}-- \\
-- \\
--\end{array}$ & $\begin{array}{l}-- \\
-- \\
--\end{array}$ \\
\hline $\begin{array}{l}3243-\text { WOM } \\
3242-\text { WOM } \\
3241-\text { WOM } \\
3239-\text { WOM } \\
3238-W O M\end{array}$ & $\begin{array}{l}3.0 \\
0.5 \\
1.0 \\
0.6 \\
1.0\end{array}$ & $\begin{array}{r}1.1 \\
8.8 \\
9.5 \\
32.4 \\
8.1\end{array}$ & $\begin{array}{r}-- \\
-- \\
1.1 \\
9.0\end{array}$ & $\begin{array}{l}-- \\
-- \\
-- \\
0.88 \\
3.4\end{array}$ \\
\hline $\begin{array}{l}3237-W O M \\
3236-W O M \\
3235-W O M \\
3234-W O M \\
3233-W O M\end{array}$ & $\begin{array}{l}2.0 \\
1.3 \\
1.0 \\
0.8 \\
1.1\end{array}$ & $\begin{array}{r}36.3 \\
7.1 \\
28.5 \\
20.0 \\
30.2\end{array}$ & $\begin{array}{l}0.50 \\
4.0 \\
6.6 \\
5.2 \\
1.6\end{array}$ & $\begin{array}{l}0.35 \\
2.8 \\
1.2 \\
2.1 \\
0.87\end{array}$ \\
\hline
\end{tabular}

\begin{tabular}{|c|c|}
\hline $\begin{array}{l}-- \\
-- \\
-- \\
--\end{array}$ & $\begin{array}{l}80.6 \\
80.9 \\
23.2 \\
75.8 \\
61.2\end{array}$ \\
\hline $\begin{array}{l}-- \\
-- \\
-- \\
--\end{array}$ & $\begin{array}{l}81.1 \\
74.9 \\
74.5 \\
76.8 \\
64.3\end{array}$ \\
\hline -- & 77.5 \\
\hline $\begin{array}{l}-- \\
-- \\
-- \\
--\end{array}$ & $\begin{array}{l}49.9 \\
25.8 \\
74.7 \\
58.0\end{array}$ \\
\hline $\begin{array}{l}-- \\
-- \\
-- \\
3.34 \\
5.52\end{array}$ & $\begin{array}{l}84.2 \\
62.1 \\
52.4 \\
11.9 \\
64.3\end{array}$ \\
\hline $\begin{array}{l}3.00 \\
4.92 \\
3.08 \\
4.40 \\
3.18\end{array}$ & $\begin{array}{r}4.7 \\
67.6 \\
20.9 \\
39.3 \\
14.7\end{array}$ \\
\hline
\end{tabular}

0.5
1.1
1.4
2.4
3.4
6.7
9.7
11.2
13.2
13.5
16.0
18.5
18.8
19.7
20.0
23.0
23.5
24.5
25.1
26.1
28.1
29.4
30.4
31.2
32.3

1.80

3.60

11.43

14.73

14.73
23.13

26.93

28.83

33. 18

37.98

39.81

46.56

55.81

63.01

67.06

70.51

73.81

78. 21

87. 71

107.15

115.25

187.85

197.08

225. 58

241. 58

274.80 
P- 83 Phosphate rock, argillaceous

P- 82 Phosphate rock and phosphatic mudstone

P- 81 Phosphate rock

P- 79 Phosphate rock, argillaceous

P- 78 Phosphate rock and phosphatic mudstone

P- 77 Phosphate rock

P- 76 Phosphate rock

P- 75 Phosphate rock, calcareous

P- 74 Phosphate rock

P- 73 Mudstone and phosphate rock

P- 72 Phosphate rock

$P-71$ Phosphate rock, argillaceous

P- 70 Mudstone

P- 69 Mudstone

P- 68 Phosphate rock, argillaceous

P- 67 Phosphate rock, argillaceous

P- 66 Mudstone, phosphatic

P- 65 Phosphate rock, argillaceous

P - 64 Mudstone

P- 63 Mudstone

P- 62 Phosphate rock, argillaceous

$\omega P-61$

P - 59

Mudstone, phosphatic

P- 58 Mudstone, phosphatic

P - 57 Phosphate rock, argillaceous

P- 56 Mudstone, phosphatic

P- 55 Mudstone, phosphatic

P- 54 Mudstone

P- 53 Mudstone, phosphatic

P- 52 Phosphate rock, argillaceous

P- 51 Mudstone, phosphatic

P- 50 Mudstone

P- 49 Mudstone

P- 48 Mudstone

P- 47 Muds tone

P- 46 Mudstone

P- 45 Mudstone

P- 44 Mudstone

P- 43 Mudstone, phosphatic

$P-42$ Mudstone

P- 41 Mudstone, phosphatic

P- 40 Phosphate rock

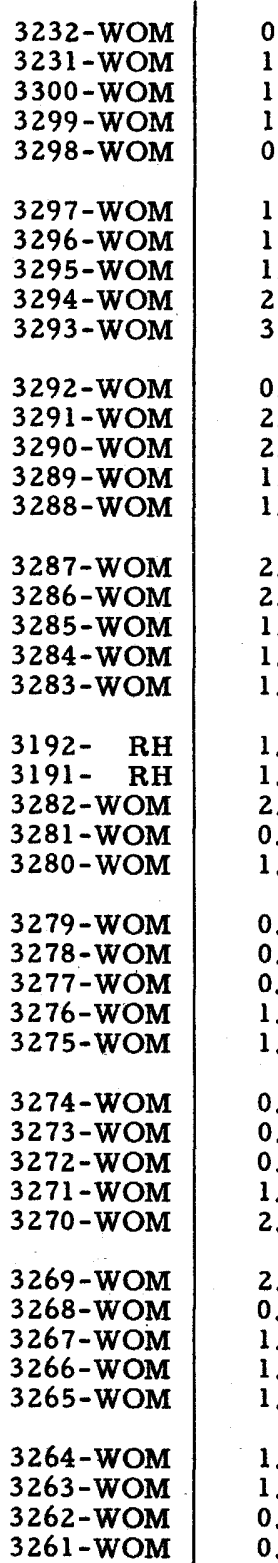

\begin{tabular}{|c|c|c|c|c|c|}
\hline $\begin{array}{l}0.8 \\
1.0 \\
1.2 \\
1.0 \\
0.7\end{array}$ & $\begin{array}{l}23.0 \\
32.5 \\
37.3 \\
33.6 \\
19.8\end{array}$ & $\begin{array}{l}4.80 \\
2.0 \\
0.40 \\
1.7 \\
4.0\end{array}$ & $\begin{array}{l}1.8 \\
1.0 \\
0.40 \\
0.93 \\
1.9\end{array}$ & $\begin{array}{l}4.90 \\
2.92 \\
2.36 \\
5.08 \\
6.56\end{array}$ & $\begin{array}{r}31.8 \\
12.5 \\
4.2 \\
8.5 \\
37.3\end{array}$ \\
\hline $\begin{array}{l}1.1 \\
1.4 \\
1.5 \\
2.4 \\
3.0\end{array}$ & $\begin{array}{l}23.6 \\
34.7 \\
35.6 \\
25.1 \\
27.2\end{array}$ & $\begin{array}{l}3.6 \\
1.0 \\
0.86 \\
2.6 \\
2.5\end{array}$ & $\begin{array}{l}1.4 \\
0.54 \\
0.52 \\
1.1 \\
1.2\end{array}$ & $\begin{array}{r}9.04 \\
5.38 \\
6.90 \\
16.54 \\
14.12\end{array}$ & $\begin{array}{r}25.8 \\
5.3 \\
3.0 \\
14.3 \\
12.9\end{array}$ \\
\hline $\begin{array}{l}0.3 \\
2.0 \\
2.8 \\
1.2 \\
1.2\end{array}$ & $\begin{array}{r}26.0 \\
23.7 \\
17.5 \\
6.9 \\
1.6\end{array}$ & $\begin{array}{c}2.6 \\
3.4 \\
4.7 \\
-- \\
--\end{array}$ & $\begin{array}{l}1.2 \\
1.5 \\
1.8 \\
-- \\
-.\end{array}$ & $\begin{array}{c}14.24 \\
15.18 \\
14.56 \\
-- \\
.-\end{array}$ & $\begin{array}{l}16.3 \\
18.8 \\
34.6 \\
63.0 \\
83.0\end{array}$ \\
\hline $\begin{array}{l}2.5 \\
2.0 \\
1.5 \\
1.1 \\
1.1\end{array}$ & $\begin{array}{r}17.0 \\
19.5 \\
16.6 \\
28.2 \\
5.7\end{array}$ & $\begin{array}{l}-- \\
-- \\
-- \\
-- \\
--\end{array}$ & $\begin{array}{l}-- \\
-- \\
-- \\
-- \\
--\end{array}$ & $\begin{array}{l}-- \\
-- \\
-- \\
--\end{array}$ & $\begin{array}{l}37.7 \\
37.8 \\
45.8 \\
22.0 \\
77.3\end{array}$ \\
\hline $\begin{array}{l}1.0 \\
1.0 \\
2.0 \\
0.8 \\
1.3\end{array}$ & $\begin{array}{r}7.6 \\
21.4 \\
10.4 \\
8.4 \\
5.7\end{array}$ & $\begin{array}{l}-- \\
-- \\
-- \\
--\end{array}$ & $\begin{array}{l}-- \\
-- \\
-- \\
--\end{array}$ & $\begin{array}{l}-- \\
-- \\
-- \\
--\end{array}$ & $\begin{array}{l}71.5 \\
34.3 \\
64.2 \\
69.2 \\
73.0\end{array}$ \\
\hline $\begin{array}{l}0.7 \\
0.7 \\
0.4 \\
1.2 \\
1.0\end{array}$ & $\begin{array}{r}13.9 \\
20.8 \\
13.6 \\
11.4 \\
3.7\end{array}$ & $\begin{array}{l}-- \\
-- \\
-- \\
--\end{array}$ & $\begin{array}{l}-- \\
-- \\
-- \\
--\end{array}$ & $\begin{array}{l}-- \\
-- \\
-- \\
--\end{array}$ & $\begin{array}{l}51.2 \\
38.2 \\
50.8 \\
54.5 \\
74.9\end{array}$ \\
\hline $\begin{array}{l}0.6 \\
0.6 \\
0.8 \\
1.0 \\
2.0\end{array}$ & $\begin{array}{r}15.6 \\
21.6 \\
12.7 \\
5.2 \\
5.3\end{array}$ & $\begin{array}{l}-- \\
-- \\
-- \\
--\end{array}$ & $\begin{array}{l}-- \\
-- \\
-- \\
--\end{array}$ & $\begin{array}{l}-- \\
-- \\
-- \\
--\end{array}$ & $\begin{array}{l}46.1 \\
33.0 \\
53.6 \\
69.1 \\
76.4\end{array}$ \\
\hline $\begin{array}{l}2.5 \\
0.4 \\
1.3 \\
1.2 \\
1.5\end{array}$ & $\begin{array}{l}4.8 \\
1.4 \\
2.4 \\
1.8 \\
5.6\end{array}$ & $\begin{array}{l}-- \\
-- \\
-- \\
--\end{array}$ & $\begin{array}{l}-- \\
-- \\
-- \\
--\end{array}$ & $\begin{array}{l}-- \\
-- \\
-- \\
--\end{array}$ & $\begin{array}{l}70.8 \\
83.7 \\
80.7 \\
84.2 \\
70.7\end{array}$ \\
\hline $\begin{array}{l}1.0 \\
1.2 \\
0.5 \\
0.8\end{array}$ & $\begin{array}{r}15.7 \\
3.9 \\
14.7 \\
26.5\end{array}$ & $\begin{array}{l}-- \\
-- \\
--\end{array}$ & $\begin{array}{l}-- \\
=- \\
--\end{array}$ & $\begin{array}{l}-- \\
-- \\
--\end{array}$ & $\begin{array}{l}41.2 \\
74.0 \\
41.3 \\
15.9\end{array}$ \\
\hline
\end{tabular}

${ }^{1}$ Fossil collection made by J. E. Smedley, Paleontology and Stratigraphv Rrnn.ch, U. S. Geological Survey.

$1,056.83$

$1,066.19$ $1,079.15$

$1,089.31$

$1,094.51$

1, 105.11

$1,117.11$

$1,117.67$

$1,120.79$

$1,122.95$

$1,131.35$

$1,147.05$

$1,151.73$

$1,159.08$ 


\begin{tabular}{|c|c|c|c|c|c|c|c|c|c|c|}
\hline \multirow[b]{2}{*}{$\begin{array}{c}\text { Bed } \\
\text { no. }\end{array}$} & \multirow[b]{2}{*}{ Rock description } & \multirow{2}{*}{$\begin{array}{l}\text { Sample } \\
\text { no. }\end{array}$} & \multirow{2}{*}{$\begin{array}{c}\text { Thickness } \\
\text { (feet) }\end{array}$} & \multicolumn{5}{|c|}{ Chemical analyses (percent) } & \multirow{2}{*}{$\begin{array}{l}\text { Cumulative } \\
\text { thickness } \\
\text { (feet) }\end{array}$} & \multirow{2}{*}{$\begin{array}{l}\text { Thickness } x \\
\text { percent } \mathrm{P}_{2} \mathrm{O}_{5} \\
\text { (cumulative) }\end{array}$} \\
\hline & & & & $\mathrm{P}_{2} \mathrm{O}_{5}$ & $\mathrm{Al}_{2} \mathrm{O}_{3}$ & $\mathrm{Fe}_{2} \mathrm{O}_{3}$ & $\begin{array}{l}\text { Loss on } \\
\text { ignition }\end{array}$ & $\begin{array}{l}\text { Acid } \\
\text { insoluble }\end{array}$ & & \\
\hline $\begin{array}{l}P-39 \\
P-38 \\
P-37 \\
P-36 \\
P-35\end{array}$ & $\begin{array}{l}\text { Mudstone, phosphatic } \\
\text { Phosphate rock, argillaceous } \\
\text { Mudstone } \\
\text { Phosphate rock } \\
\text { Mudstone }\end{array}$ & $\begin{array}{l}3230-W O M \\
3229-W O M \\
3228-W O M \\
3227-W O M \\
3226-W O M\end{array}$ & $\begin{array}{l}0.5 \\
1.5 \\
2.0 \\
2.0 \\
2.0\end{array}$ & $\begin{array}{r}16.4 \\
20.3 \\
1.4 \\
28.8 \\
9.9\end{array}$ & $\begin{array}{l}-- \\
-- \\
-- \\
--\end{array}$ & $\begin{array}{l}-- \\
-- \\
-- \\
--\end{array}$ & $\begin{array}{l}-- \\
-- \\
-- \\
--\end{array}$ & $\begin{array}{l}44.9 \\
36.0 \\
87.1 \\
16.7 \\
62.7\end{array}$ & $\begin{array}{l}88.1 \\
89.6 \\
91.6 \\
93.6 \\
95.6\end{array}$ & $\begin{array}{l}1,188.48 \\
1,218.93 \\
1,221.73 \\
1,279.33 \\
1,299.13\end{array}$ \\
\hline $\begin{array}{l}P-34 \\
P-33 \\
P-32 \\
P=31 \\
P=30\end{array}$ & $\begin{array}{l}\text { Phosphate rock, argillaceous } \\
\text { Mudstone } \\
\text { Mudstone, phosphatic } \\
\text { Mudstone } \\
\text { Mudstone, phosphatic }\end{array}$ & $\begin{array}{l}3225-\text { WOM } \\
3224-\text { WOM } \\
3223-\text { WOM } \\
3222-\text { WOM } \\
3221-\text { WOM }\end{array}$ & $\begin{array}{l}1.5 \\
0.6 \\
1.5 \\
1.0 \\
2.0\end{array}$ & $\begin{array}{r}20.5 \\
7.4 \\
11.1 \\
1.9 \\
8.7\end{array}$ & $\begin{array}{l}-- \\
-- \\
-- \\
--\end{array}$ & $\begin{array}{l}-- \\
-- \\
-- \\
--\end{array}$ & $\begin{array}{l}-- \\
-- \\
-- \\
--\end{array}$ & $\begin{array}{l}34.9 \\
68.2 \\
56.1 \\
83.7 \\
62.5\end{array}$ & $\begin{array}{r}97.1 \\
97.7 \\
99.2 \\
100.2 \\
102.2\end{array}$ & $\begin{array}{l}1,329.88 \\
1,334.32 \\
1,350.97 \\
1,352.87 \\
1,370.27\end{array}$ \\
\hline $\begin{array}{l}P-29 \\
P-28 \\
P=27 \\
P=26 \\
P=25\end{array}$ & $\begin{array}{l}\text { Mudstone, phosphatic, calcareous } \\
\text { Mudstone, calcareous, phosphatic } \\
\text { Mudstone, calcareous } \\
\text { Mudstone, calcareous } \\
\text { Mudstone }\end{array}$ & $\begin{array}{ll}3220- & \text { RH } \\
3219- & \text { RH } \\
3218- & \text { RH } \\
3217- & \text { RH } \\
3216- & \text { RH }\end{array}$ & $\begin{array}{l}2.5 \\
3.0 \\
1.5 \\
0.8 \\
0.7\end{array}$ & $\begin{array}{r}11.5 \\
9.6 \\
3.7 \\
1.4 \\
2.3\end{array}$ & $\begin{array}{l}-- \\
-- \\
-- \\
--\end{array}$ & $\begin{array}{l}-- \\
-- \\
-- \\
--\end{array}$ & $\begin{array}{l}-- \\
-- \\
-- \\
--\end{array}$ & $\begin{array}{l}42.1 \\
40.5 \\
62.5 \\
58.0 \\
70.9\end{array}$ & $\begin{array}{l}104.7 \\
107.7 \\
109.2 \\
110.0 \\
110.7\end{array}$ & $\begin{array}{l}1,399.02 \\
1,427.82 \\
1,433.37 \\
1,434.49 \\
1,436.10\end{array}$ \\
\hline $\begin{array}{l}P-24 \\
P=23 \\
P-22 \\
P-21 \\
P-20\end{array}$ & $\begin{array}{l}\text { Mudstone } \\
\text { Chert } \\
\text { Mudstone } \\
\text { Mudstone } \\
\text { Mudstone, phosphatic }\end{array}$ & $\begin{array}{ll}3215- & \text { RH } \\
3214- & \text { RH } \\
3213- & \text { RH } \\
3212- & \text { RH } \\
3211- & \text { RH }\end{array}$ & $\begin{array}{l}2.5 \\
0.4 \\
0.7 \\
2.3 \\
1.5\end{array}$ & $\begin{array}{l}1.1 \\
1.4 \\
2.4 \\
1.9 \\
8.2\end{array}$ & $\begin{array}{l}-- \\
-- \\
-- \\
--\end{array}$ & $\begin{array}{l}-- \\
-- \\
-- \\
--\end{array}$ & $\begin{array}{l}-- \\
-- \\
-- \\
--\end{array}$ & $\begin{array}{l}80.4 \\
88.7 \\
83.5 \\
79.1 \\
60.2\end{array}$ & $\begin{array}{l}113.2 \\
113.6 \\
114.3 \\
116.6 \\
118.1\end{array}$ & $\begin{array}{l}1,438.85 \\
1,439.41 \\
1,441.09 \\
1,445.46 \\
1,457.76\end{array}$ \\
\hline $\begin{array}{l}P-19 \\
P=18 \\
P=17 \\
P-16 \\
P=15\end{array}$ & $\begin{array}{l}\text { Mudstone } \\
\text { Mudstone } \\
\text { Mudstone, phosphatic } \\
\text { Mudstone } \\
\text { Mudstone }\end{array}$ & $\begin{array}{ll}3210- & \text { RH } \\
3209- & \text { RH } \\
3208- & \text { RH } \\
3207- & \text { RH } \\
3206- & \text { RH }\end{array}$ & $\begin{array}{l}1.5 \\
1.5 \\
1.0 \\
0.9 \\
1.0\end{array}$ & $\begin{array}{l}1.5 \\
4.7 \\
8.7 \\
0.8 \\
3.8\end{array}$ & $\begin{array}{l}-- \\
-- \\
-- \\
--\end{array}$ & $\begin{array}{l}-- \\
-- \\
-- \\
--\end{array}$ & $\begin{array}{l}-- \\
-- \\
-- \\
-- \\
--\end{array}$ & $\begin{array}{l}86.7 \\
74.2 \\
61.1 \\
87.5 \\
77.2\end{array}$ & $\begin{array}{l}119.6 \\
121.1 \\
122.1 \\
123.0 \\
124.0\end{array}$ & $\begin{array}{l}1,460.01 \\
1,467.06 \\
1,475.76 \\
1,476.48 \\
1,480.28\end{array}$ \\
\hline $\begin{array}{l}P-14 \\
P-13 \\
P-12 \\
P-11 \\
P=10\end{array}$ & $\begin{array}{l}\text { Mudstone } \\
\text { Covered } \\
\text { Limestone } \\
\text { Phosphate rock, argillaceous } \\
\text { Phosphate rock, calcareous, argillaceous }\end{array}$ & $\begin{array}{rr}3205- & \text { RH } \\
-- & \\
3203- & \text { RH } \\
3202- & \text { RH } \\
3201- & \text { RH }\end{array}$ & $\begin{array}{r}1.3 \\
40 . \\
1.0 \\
1.8 \\
0.8\end{array}$ & $\begin{array}{r}1.8 \\
-- \\
2.9 \\
21.1 \\
17.2\end{array}$ & $\begin{array}{l}-- \\
-- \\
-- \\
--\end{array}$ & $\begin{array}{l}-- \\
-- \\
-- \\
--\end{array}$ & $\begin{array}{l}-- \\
-- \\
-- \\
--\end{array}$ & $\begin{array}{l}81.9 \\
-- \\
15.1 \\
32.4 \\
25.7\end{array}$ & $\begin{array}{l}125.3 \\
165.3 \\
166.3 \\
168.1 \\
168.9\end{array}$ & $\begin{array}{r}1,482.62 \\
2.90 * \\
40.88 \\
54.64\end{array}$ \\
\hline $\begin{array}{l}P-\quad 9 \\
P-\quad 8 \\
P=\quad 7 \\
P-\quad 6 \\
P=\quad 5\end{array}$ & $\begin{array}{l}\text { Limestone, phosphatic, argillaceous } \\
\text { Limestone, phosphatic } \\
\text { Phosphate rock } \\
\text { Phosphate rock } \\
\text { Phosphate rock }\end{array}$ & $\begin{array}{ll}3200- & \text { RH } \\
3199- & \text { RH } \\
3198- & \text { RH } \\
3197- & \text { RH } \\
3196- & \text { RH }\end{array}$ & $\begin{array}{l}0.7 \\
2.4 \\
1.5 \\
1.0 \\
1.5\end{array}$ & $\begin{array}{l}10.7 \\
11.4 \\
33.4 \\
35.2 \\
36.2\end{array}$ & \begin{tabular}{l|}
-- \\
-- \\
0.99 \\
0.72 \\
0.26
\end{tabular} & $\begin{array}{l}-- \\
0.46 \\
0.59 \\
0.35\end{array}$ & $\begin{array}{l}-- \\
-- \\
6.54 \\
6.54 \\
5.28\end{array}$ & $\begin{array}{r}20.3 \\
8.9 \\
6.3 \\
3.3 \\
1.8\end{array}$ & $\begin{array}{l}169.6 \\
172.0 \\
173.5 \\
174.5 \\
176.0\end{array}$ & $\begin{array}{r}62.13 \\
89.49 \\
139.59 \\
174.79 \\
229.09\end{array}$ \\
\hline P- 4 & Phosphate rock and phosphatic mudstone & 3195- RH & 1.5 & 27.1 & - & -- & -- & 21.1 & 177.5 & 269.74 \\
\hline
\end{tabular}




\begin{tabular}{|c|c|c|c|c|c|c|c|c|c|c|}
\hline $\begin{array}{ll}P- & 3 \\
P- & 2 \\
P- & 1\end{array}$ & $\begin{array}{l}\text { Limestone, argillaceous } \\
\text { Limestone, argillaceous } \\
\text { Covered }\end{array}$ & $\begin{array}{cc}3194- & \text { RH } \\
3193- & \text { RH } \\
-- & \end{array}$ & $\begin{array}{l}0.5 \\
1.5 \\
2.0(?)\end{array}$ & $\begin{array}{l}1.7 \\
0.5 \\
--\end{array}$ & $\begin{array}{l}-- \\
--\end{array}$ & $\begin{array}{l}-- \\
--\end{array}$ & $\begin{array}{ll}-- \\
--\end{array}$ & $\begin{array}{c}32.5 \\
36.5 \\
--\end{array}$ & $\begin{array}{l}178.0 \\
179.5 \\
181.5\end{array}$ & $\begin{array}{l}270.59 \\
271.34 * * \\
--\end{array}$ \\
\hline
\end{tabular}

Phosphoria-Wells contact not exposed; covered interval probably not more than 3 feet thick.

Wells formation - not exposed

* Cumulative data incomplete due to missing information. Computations start from zero after interruption.

** Note incompleteness of cumulative data. 
PARIS CANYON, IDAHO. LOT NO. 1266.

Phosphatic shale member of Phosphoria formation sampled in bulldozer trench on north side of Paris Canyon, sec. 8 , T. 14 S., R. 43 E.,

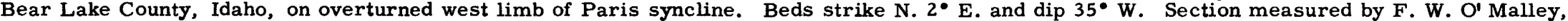
R. A. Harris, and D. F. Davidson and sampled by R. G. Waring and Harris in September 1948 . Samples analyzed for $P_{2} O_{5}$ and acid insoluble by

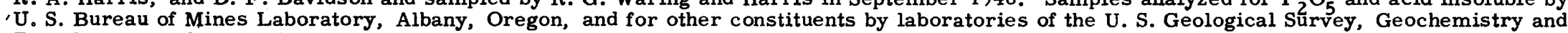
Petrology Branch, Washington, D. C.

\begin{tabular}{|c|c|c|c|c|c|c|c|c|c|c|}
\hline \multirow{2}{*}{$\begin{array}{l}\text { Bed } \\
\text { no. }\end{array}$} & \multirow[b]{2}{*}{ Rock description } & \multirow{2}{*}{$\begin{array}{c}\text { Sample } \\
\text { no. }\end{array}$} & \multirow{2}{*}{$\begin{array}{c}\text { Thickness } \\
\text { (feet) }\end{array}$} & \multicolumn{5}{|c|}{ Chemical analyses (percent) } & \multirow{2}{*}{$\begin{array}{c}\text { Cumulative } \\
\text { thickness } \\
\text { (feet) }\end{array}$} & \multirow{2}{*}{$\begin{array}{l}\text { Thickness } \mathrm{x} \\
\text { percent } \mathrm{P}_{2} \mathrm{O}_{5} \\
\text { (cumulative) }\end{array}$} \\
\hline & & & & $\mathrm{P}_{2} \mathrm{O}_{5}$ & $\mathrm{Al}_{2} \mathrm{O}_{3}$ & $\mathrm{Fe}_{2} \mathrm{O}_{3}$ & $\begin{array}{l}\text { Loss on } \\
\text { ignition }\end{array}$ & $\begin{array}{c}\text { Acid } \\
\text { insoluble }\end{array}$ & & \\
\hline
\end{tabular}

Rex member of Phosphoria formation-basal beds only

\begin{tabular}{|c|c|c|c|c|c|c|c|c|c|c|}
\hline $\begin{array}{l}\mathbf{R}- \\
\mathbf{R}- \\
\mathbf{R}-\end{array}$ & $\begin{array}{l}\text { Chert } \\
\text { Chert } \\
\text { Chert }\end{array}$ & $\begin{array}{l}3180-W O M \\
3179-W O M \\
3178-W O M\end{array}$ & $\begin{array}{l}1.0 \\
1.1 \\
3.0\end{array}$ & $\begin{array}{l}0.8 \\
2.3 \\
1.3\end{array}$ & $\begin{array}{l}-- \\
--\end{array}$ & $\begin{array}{l}-- \\
--\end{array}$ & $\begin{array}{l}-- \\
--\end{array}$ & $\begin{array}{r}94.5 \\
.89 .0 \\
93.1\end{array}$ & $\begin{array}{l}1.0 \\
2.1 \\
5.1\end{array}$ & $\begin{array}{l}0.80 \\
3.33 \\
7.23\end{array}$ \\
\hline
\end{tabular}

$\stackrel{\omega}{\oplus}$

\begin{tabular}{|c|c|c|}
\hline $\begin{array}{l}P-119 \\
P-118 \\
P-117 \\
P-116 \\
P-115\end{array}$ & $\begin{array}{l}\text { Mudstone and phosphatic mudstone } \\
\text { Mudstone } \\
\text { Mudstone } \\
\text { Mudstone; fos. col. no. } 48-J E S-367^{1} \\
\text { Mudstone }\end{array}$ & $\begin{array}{l}3177-\text { WOM } \\
3176-\text { WOM } \\
3175-\text { WOM } \\
3174-\text { WOM } \\
3173-\text { WOM }\end{array}$ \\
\hline $\begin{array}{l}P-114 \\
P-11 \\
P-11 \\
P-11 \\
P-11\end{array}$ & $\begin{array}{l}\text { Mudstone } \\
\text { Phosphate rock } \\
\text { Mudstone } \\
\text { Phosphate rock, argillaceous } \\
\text { Mudstone }\end{array}$ & $\begin{array}{l}3172-\text { WOM } \\
3171-\text { WOM } \\
3170-\text { WOM } \\
3169-\text { WOM } \\
3168-\text { WOM }\end{array}$ \\
\hline $\begin{array}{l}P-109 \\
P-108 \\
P-107 \\
P-106 \\
P-105\end{array}$ & $\begin{array}{l}\text { Phosphate rock } \\
\text { Phosphate rock, argillaceous } \\
\text { Phosphate rock } \\
\text { Phosphate rock, argillaceous; fos. col. } \\
\text { no. } 48 \text {-JES- } 366 \\
\text { Phosphate rock }\end{array}$ & $\begin{array}{l}3167-W O \\
3166-W O \\
3165-W O \\
3164-W O \\
3163-W O\end{array}$ \\
\hline $\begin{array}{l}P-104 \\
P-103 \\
P-102 \\
P-101 \\
P-100\end{array}$ & $\begin{array}{l}\text { Phosphate rock } \\
\text { Limestone } \\
\text { Phosphate rock } \\
\text { Phosphate rock, calcareous, argillaceous } \\
\text { Phosphate rock, argillaceous, calcareous }\end{array}$ & $\begin{array}{l}3162-W O \\
3161-W O \\
3160-W O \\
3159-W O \\
3158-W O\end{array}$ \\
\hline $\begin{array}{l}P-99 \\
P-98 \\
P=97 \\
P=96 \\
P=95\end{array}$ & $\begin{array}{l}\text { Phosphate rock and argillaceous } \\
\text { calcareous phosphate rock } \\
\text { Phosphate rock, argillaceous, calcareous } \\
\text { Limestone } \\
\text { Limestone, argillaceous } \\
\text { Mudstone }\end{array}$ & $\begin{array}{l}3157-W O \\
3156-W O \\
3155-W O \\
3154-W O \\
3153-W O\end{array}$ \\
\hline
\end{tabular}

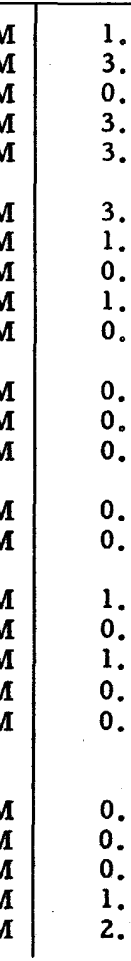

\begin{tabular}{l|r|l|l|l|}
\hline 1.0 & 3.2 & -- & -- & -- \\
3.0 & 3.5 & -- & -- & -- \\
0.7 & 0.8 & -- & -- & -- \\
3.4 & 1.2 & -- & -- & -- \\
3.0 & 5.8 & -- & -- & -- \\
3.0 & 2.3 & -- & -- & -- \\
1.7 & 35.6 & 0.92 & 0.74 & 5.54 \\
0.9 & 7.4 & 8.2 & 3.06 & 6.76 \\
1.0 & 21.6 & 2.7 & 1.4 & 6.66 \\
0.4 & 5.3 & 1.2 & 0.77 & 5.90 \\
& & & & \\
0.8 & 34.7 & 3.5 & 1.4 & 10.54 \\
0.5 & 25.5 & 0.76 & 0.66 & 3.48 \\
0.6 & 33.7 & 9.2 & 3.1 & 6.80 \\
0.9 & 21.7 & 4.9 & 2.2 & 6.48 \\
0.5 & 31.6 & 9.9 & 4.1 & 10.56 \\
1.0 & 30.5 & 2.1 & 1.1 & 12.56 \\
0.4 & 4.8 & 1.4 & 0.69 & 39.82 \\
1.1 & 31.8 & 1.1 & 0.60 & 13.10 \\
0.9 & 22.8 & 3.0 & 1.4 & 15.64 \\
0.6 & 18.5 & 4.8 & 2.8 & 16.56 \\
& & & & \\
0.6 & 26.7 & 1.7 & 0.74 & 13.30 \\
0.8 & 20.2 & 4.0 & 1.7 & 17.46 \\
0.6 & 2.8 & -- & -- & -- \\
1.3 & 2.9 & -- & -- & -- \\
2.3 & 3.3 & -- & -- & -- \\
& & & &
\end{tabular}

\begin{tabular}{r|}
74.4 \\
73.8 \\
77.9 \\
77.6 \\
68.7 \\
75.5 \\
5.6 \\
65.4 \\
33.0 \\
62.8 \\
6.2 \\
23.6 \\
6.9 \\
30.0 \\
9.4 \\
7.4 \\
9.3 \\
5.2 \\
20.1 \\
29.3 \\
6 \\
15.3 \\
25.2 \\
11.5 \\
35.4 \\
65.0 \\
\end{tabular}

1.0
4.0
4.7
8.1
11.1
14.1
15.8
16.7
17.7
18.1
18.9
19.4
20.0
20.9
21.4
22.4
22.8
23.9
24.8
25.4
26.0
26.8
27.4
28.7
31.0

3. 20

13. 70

14.26

18.34

35.74

42.64

103.16

109.82

131.42

133.54

161.30

174.05

194.27

213.80

229.60

260.10

262.02

297.00

317.52

328.62

344.64

360.80

362.48

366.25

373.84 


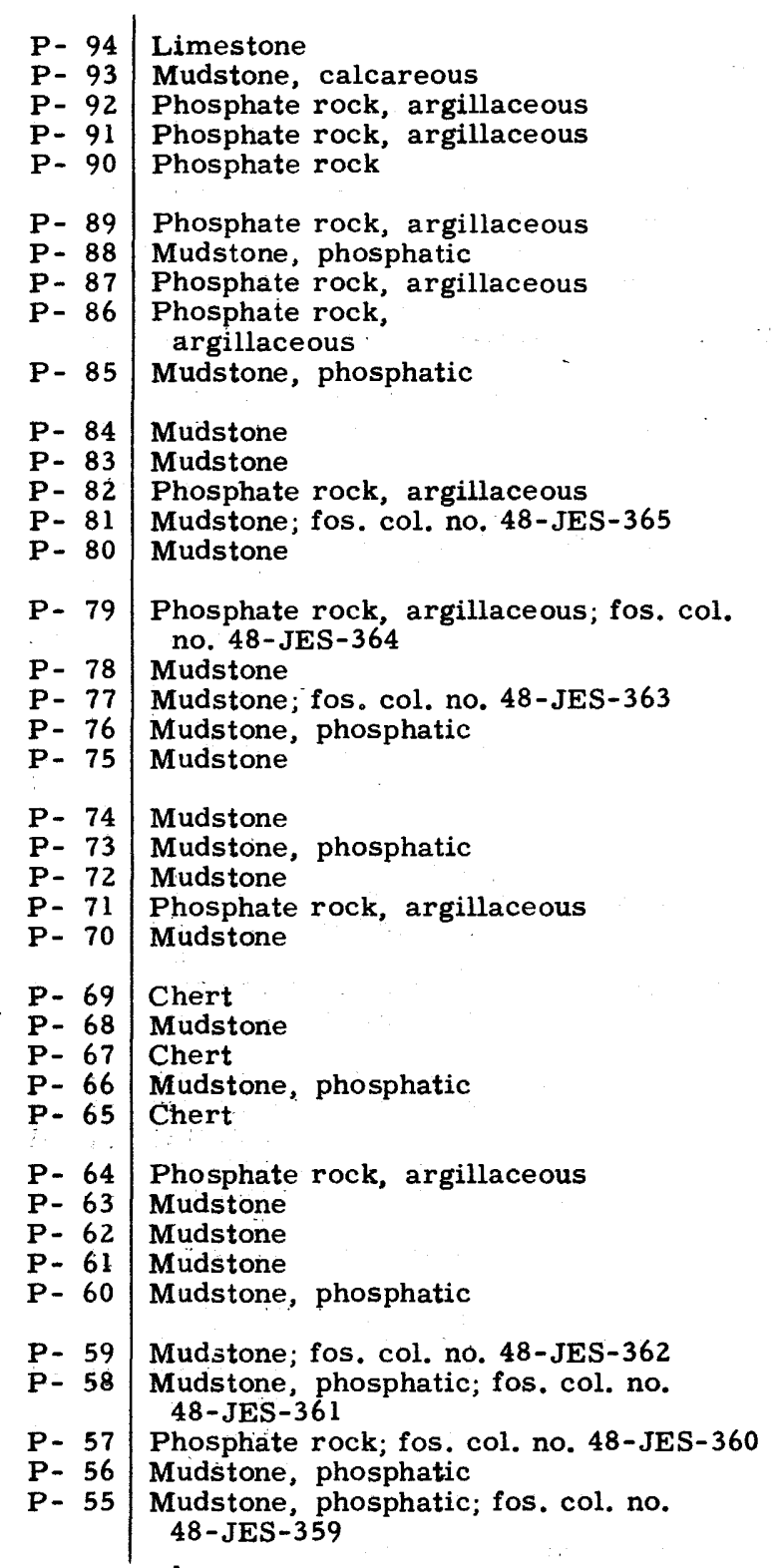

\begin{tabular}{|l|} 
3152-WOM \\
$3151-$ WOM \\
$3150-$ WOM \\
$3149-$ WOM \\
$3148-$ WOM \\
$3147-$ WOM \\
$3146-$ WOM \\
$3145-$ WOM \\
$3144-$ WOM \\
$3143-$ WOM \\
$3142-$ WOM \\
$3141-$ WOM \\
$3140-$ WOM \\
$3139-$ WOM \\
$3138-$ WOM \\
$3137-$ WOM \\
$3136-$ WOM \\
$3135-$ WOM \\
$3134-$ WOM \\
$3133-$ WOM \\
$3132-$ WOM \\
$3131-$ WOM \\
$3130-$ WOM \\
$3129-$ WOM \\
$3128-$ WOM \\
$3127-$ WOM \\
$3126-$ WOM \\
$3125-$ WOM \\
$3124-$ WOM \\
$3123-$ WOM \\
$3122-$ WOM \\
$3121-$ WOM \\
$3120-$ WOM \\
$3119-$ WOM \\
$3118-$ WOM \\
$3117-$ WOM \\
$3116-$ WOM \\
$3115-$ WOM \\
$3114-$ WOM \\
\\
$3113-$ WOM
\end{tabular}

\begin{tabular}{|c|c|c|c|}
\hline $\begin{array}{l}3.5 \\
0.4 \\
0.6 \\
0.5 \\
0.6\end{array}$ & $\begin{array}{r}0.6 \\
3.8 \\
22.5 \\
18.8 \\
32.0\end{array}$ & $\begin{array}{l}-- \\
-- \\
-- \\
--\end{array}$ & $\begin{array}{l}-- \\
-- \\
-- \\
--\end{array}$ \\
\hline $\begin{array}{l}0.7 \\
2.2 \\
3.7\end{array}$ & $\begin{array}{l}16.5 \\
10.9 \\
13.1\end{array}$ & $\begin{array}{l}-- \\
--\end{array}$ & $\begin{array}{l}-- \\
--\end{array}$ \\
\hline $\begin{array}{l}2.7 \\
2.8\end{array}$ & $\begin{array}{l}18.5 \\
14.8\end{array}$ & -- & $=$ \\
\hline $\begin{array}{l}0.6 \\
1.2 \\
0.8 \\
3.3 \\
3.0\end{array}$ & $\begin{array}{r}6.3 \\
2.5 \\
21.6 \\
5.3 \\
4.9\end{array}$ & $\begin{array}{l}-- \\
-- \\
-- \\
--\end{array}$ & $\begin{array}{l}-- \\
=- \\
-- \\
--\end{array}$ \\
\hline $\begin{array}{l}1.3 \\
0.8 \\
2.4 \\
0.5 \\
1.0\end{array}$ & $\begin{array}{r}18.1 \\
7.0 \\
2.1 \\
10.1 \\
5.5\end{array}$ & $\begin{array}{l}-- \\
-- \\
-- \\
--\end{array}$ & $\begin{array}{l}-- \\
-- \\
--\end{array}$ \\
\hline $\begin{array}{l}3.0 \\
0.9 \\
4.0 \\
0.4 \\
0.9\end{array}$ & $\begin{array}{r}3.1 \\
8.5 \\
4.8 \\
19.6 \\
1.6\end{array}$ & $\begin{array}{l}-- \\
-- \\
-- \\
--\end{array}$ & $\begin{array}{l}-- \\
-- \\
-- \\
--\end{array}$ \\
\hline $\begin{array}{l}0.7 \\
2.0 \\
5.0 \\
0.6 \\
1.0\end{array}$ & $\begin{array}{r}3.0 \\
1.2 \\
0.5 \\
13.9 \\
1.6\end{array}$ & $\begin{array}{l}-- \\
-- \\
-- \\
--\end{array}$ & $\begin{array}{l}-- \\
-- \\
--\end{array}$ \\
\hline $\begin{array}{l}0.6 \\
1.7 \\
4.2 \\
3.4 \\
0.7\end{array}$ & $\begin{array}{r}19.7 \\
10.5 \\
3.6 \\
2.0 \\
16.5\end{array}$ & $\begin{array}{l}-- \\
-- \\
--\end{array}$ & $\begin{array}{l}=- \\
=- \\
-- \\
--\end{array}$ \\
\hline 2.2 & 2. 8 & -- & -- \\
\hline $\begin{array}{l}2.1 \\
0.7 \\
1.4\end{array}$ & $\begin{array}{r}14.7 \\
26.7 \\
8.4\end{array}$ & $=$ & $\begin{array}{l}-- \\
--\end{array}$ \\
\hline 0.9 & 10.6 & -- & $\because$ \\
\hline
\end{tabular}

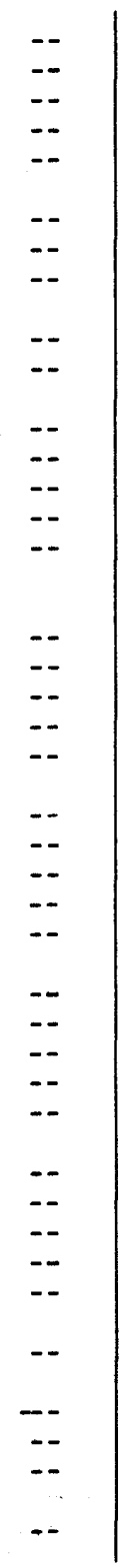

\begin{tabular}{|c|}
\hline $\begin{array}{r}13.2 \\
53.0 \\
28.4 \\
32.0 \\
9.3\end{array}$ \\
\hline $\begin{array}{l}20.7 \\
27.5 \\
25.1\end{array}$ \\
\hline $\begin{array}{l}29.2 \\
42.7\end{array}$ \\
\hline $\begin{array}{l}61.7 \\
75.4 \\
30.2 \\
71.9 \\
76.8\end{array}$ \\
\hline $\begin{array}{l}33.4 \\
65.2 \\
80.5 \\
53.4 \\
70.4\end{array}$ \\
\hline $\begin{array}{l}78.9 \\
63.2 \\
74.0 \\
42.7 \\
83.5\end{array}$ \\
\hline $\begin{array}{l}86.8 \\
80.0 \\
95.0 \\
45.4 \\
91.7\end{array}$ \\
\hline $\begin{array}{l}35.7 \\
49.8 \\
78.8 \\
79.5 \\
47.4\end{array}$ \\
\hline 80.0 \\
\hline $\begin{array}{l}51.4 \\
18.5 \\
63.3\end{array}$ \\
\hline 61.3 \\
\hline
\end{tabular}

34.5

34. 9

35.5

36.0

36.6

37.3

39.5

43.2

45. 9

48. 7

49.3

50.5

54.6
57.6

58.9

59.7

62.1

62.6

63.6

66.6

67.5

71.5

71.9

72. 8

73. 5

75.5

80.5

81.1

82.1

82. 7

84.4

88.6

92.0
92.7

94.9

94.9

97.0
97.7

99.1

375.94

377.46

390.96

400.36
419.56

431.11

455.09

503.56

553.51

594.95

598.73

601.73

619.01

636.50

651.20

674.73

680.33

685.37

690.42

695.92

705.22

712.87

732.07

739.91

741.35

743.45

745.85

748.35

756.69

758.29

770.11

787.96

803.08

809.88

821.43

827.59

858.46

877.15

888.91

1 Fossil collection made by J. E. Smedley, Paleontology and Stratigraphy Branch, U. S. Geological Survey.

100.00

898.45 


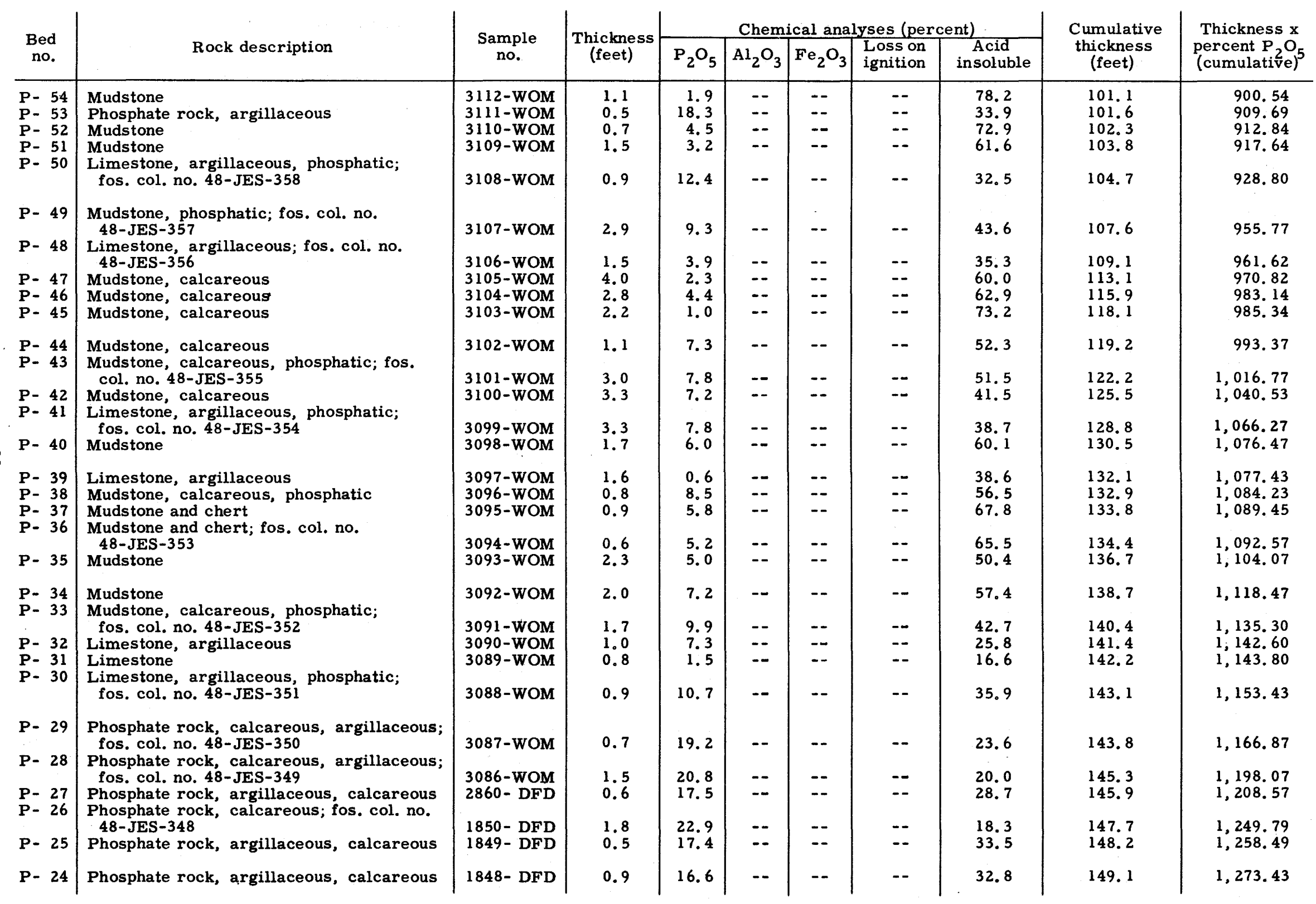


\begin{tabular}{l|l} 
P- 23 & Phosphate rock, argillaceous \\
P- 22 & Phosphate rock, calcareous
\end{tabular}

P- 22 Phosphate rock, calcareous

P- 20 Phosphate rock, calcareous

P- 19 Mudstone and calcareous phosphate rock

P- 18 Phosphate rock, argillaceous

P- 17 Phosphate rock, argillaceous

P- 16 Mudstone, phosphatic

P- 15 Mudstone, phosphatic

P- 14 Mudstone

P- 13 Phosphate rock

P- 12 Phosphate rock, argillaceous

P- 11 Mudstone, phosphatic

P- 10 Mudstone, phosphatic

P- 9 Phosphate rock, argillaceous; fos. col.

no. 48-JES-347

P- 8 Phosphate rock, argillaceous; fos. col.

P- 7 Phosphate rock, argillaceous

P- 6 Phosphate rock

P- 5 Phos phate rock

P- 4 Mudstone, phosphatic

P- 3 Mudstone

P- 2 Mudstone, calcareous

P- 1 Phosphate rock

\begin{tabular}{|c|c|c|c|c|c|c|c|c|}
\hline $\begin{array}{l}1847-\text { DFD } \\
1846-\text { DFD } \\
1845-\text { DFD } \\
1844-\text { DFD } \\
1843 \text { - DFD }\end{array}$ & $\begin{array}{l}0.7 \\
1.1 \\
0.6 \\
1.1 \\
1.4\end{array}$ & $\begin{array}{l}20.4 \\
25.8 \\
21.9 \\
23.9 \\
14.8\end{array}$ & $\begin{array}{l}-- \\
-- \\
--\end{array}$ & $\begin{array}{l}-- \\
-- \\
-- \\
--\end{array}$ & $\begin{array}{l}-- \\
-- \\
-- \\
--\end{array}$ & $\begin{array}{l}29.5 \\
11.7 \\
16.8 \\
16.8 \\
40.8\end{array}$ & $\begin{array}{l}149.8 \\
150.9 \\
151.5 \\
152.6 \\
154.0\end{array}$ & $\begin{array}{l}1,287.71 \\
1,316.09 \\
1,329.23 \\
1,355.52 \\
1,376.24\end{array}$ \\
\hline $\begin{array}{l}\text { 1842- DFD } \\
3085-\text { WOM } \\
3084-W O M \\
3083-W O M \\
3082-W O M\end{array}$ & $\begin{array}{l}0.5 \\
0.5 \\
1.0 \\
3.5 \\
1.1\end{array}$ & $\begin{array}{r}17.5 \\
17.5 \\
10.4 \\
8.4 \\
2.1\end{array}$ & $\begin{array}{l}-- \\
-- \\
--\end{array}$ & $\begin{array}{l}-- \\
-- \\
-- \\
--\end{array}$ & $\begin{array}{l}-- \\
-- \\
-- \\
--\end{array}$ & $\begin{array}{l}36.2 \\
36.9 \\
54.4 \\
63.6 \\
77.0\end{array}$ & $\begin{array}{l}154.5 \\
155.0 \\
156.0 \\
159.5 \\
160.6\end{array}$ & $\begin{array}{l}1,384.99 \\
1,393.74 \\
1,404.14 \\
1,433.54 \\
1,435.85\end{array}$ \\
\hline $\begin{array}{l}3081-W O M \\
3070-W O M \\
3069-W O M \\
3068-W O M\end{array}$ & $\begin{array}{l}0.7 \\
1.5 \\
1.5 \\
1.0\end{array}$ & $\begin{array}{r}29.0 \\
20.8 \\
9.4 \\
13.5\end{array}$ & $\begin{array}{l}-- \\
-- \\
-- \\
--\end{array}$ & $\begin{array}{l}-- \\
-- \\
--\end{array}$ & $\begin{array}{l}-- \\
-- \\
--\end{array}$ & $\begin{array}{l}17.0 \\
36.4 \\
59.7 \\
52.3\end{array}$ & $\begin{array}{l}161.3 \\
162.8 \\
164.3 \\
165.3\end{array}$ & $\begin{array}{l}1,456.15 \\
1,487.35 \\
1,501.45 \\
1,514.95\end{array}$ \\
\hline 3067-WOM & 2.3 & 17.1 & -- & -- & - & 44.8 & 167.6 & $1,554,28$ \\
\hline $\begin{array}{ll}3080- & \text { RH } \\
3079- & \text { RH } \\
3078- & \text { RH } \\
3077- & \text { RH } \\
3076- & \text { RH }\end{array}$ & $\begin{array}{l}0.8 \\
0.6 \\
2.2 \\
2.6 \\
0.6\end{array}$ & $\begin{array}{l}19.8 \\
23.5 \\
32.7 \\
33.8 \\
13.8\end{array}$ & $\begin{array}{l}-- \\
-- \\
1.4 \\
1.1 \\
--\end{array}$ & $\begin{array}{l}-- \\
-- \\
0.59 \\
0.51 \\
--\end{array}$ & $\begin{array}{c}-- \\
-- \\
5.60 \\
4.92 \\
--\end{array}$ & $\begin{array}{r}32.2 \\
23.9 \\
6.3 \\
5.1 \\
49.7\end{array}$ & $\begin{array}{l}168.4 \\
169.0 \\
171.2 \\
173.8 \\
174.4\end{array}$ & $\begin{array}{l}1,570.12 \\
1,584.22 \\
1,656.16 \\
1,744.04 \\
1,752.32\end{array}$ \\
\hline $\begin{array}{ll}3075- & \text { RH } \\
3074- & \text { RH } \\
3073- & \text { RH }\end{array}$ & $\begin{array}{l}2.5 \\
1.1 \\
0.3\end{array}$ & $\begin{array}{r}2.4 \\
0.7 \\
28.7\end{array}$ & $\begin{array}{l}-- \\
--\end{array}$ & $\begin{array}{l}-- \\
--\end{array}$ & $=-$ & $\begin{array}{r}57.7 \\
58.0 \\
7.5\end{array}$ & $\begin{array}{l}176.9 \\
178.0 \\
178.3\end{array}$ & $\begin{array}{l}1,758.32 \\
1,759.09 \\
1,767.70\end{array}$ \\
\hline
\end{tabular}

Wells formation - top beds only

\begin{tabular}{|c|c|c|c|c|c|c|c|c|c|c|}
\hline $\begin{array}{l}C w-1 \\
C w-2\end{array}$ & $\begin{array}{l}\text { Dolomite, argillaceous, calcareous } \\
\text { Dolomite, calcareous, argillaceous }\end{array}$ & $\begin{array}{ll}3072- & \text { RH } \\
3071- & \text { RH }\end{array}$ & $\begin{array}{l}4.0 \\
2.7\end{array}$ & $\begin{array}{l}5.4 \\
1.0\end{array}$ & -- & -- & -- & $\begin{array}{l}29.5 \\
22.8\end{array}$ & $\begin{array}{l}4.0 \\
6.7\end{array}$ & $\begin{array}{l}21.60 \\
24.30\end{array}$ \\
\hline
\end{tabular}


BLOOMINGTON CANYON, IDAHO. LOT NO. 1272.

Phosphatic shale member of Phosphoria formation cored in diamond drill hole no. 1 on north slope of Bloomington Canyon, sec. 21 , T. 14 S.

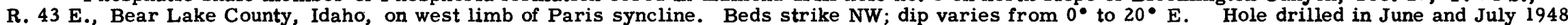

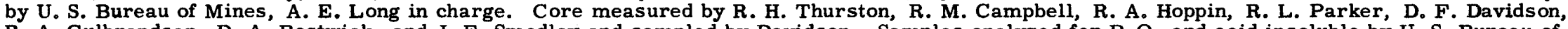

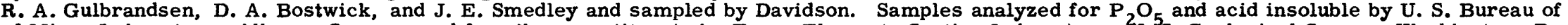

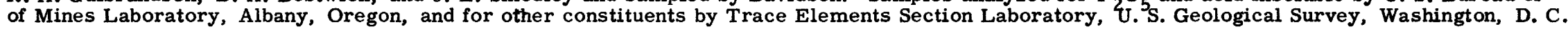

\begin{tabular}{|c|c|c|c|c|c|c|c|c|c|c|}
\hline \multirow{2}{*}{$\begin{array}{l}\text { Bed } \\
\text { no. }\end{array}$} & \multirow[b]{2}{*}{ Rock description } & \multirow{2}{*}{$\begin{array}{c}\text { Sample } \\
\text { no. }\end{array}$} & \multirow{2}{*}{$\begin{array}{c}\text { Thickness } \\
\text { (feet) }\end{array}$} & \multicolumn{5}{|c|}{ Chemical analyses (percent) } & \multirow{2}{*}{$\begin{array}{c}\text { Cumulative } \\
\text { thickness } \\
\text { (feet) }\end{array}$} & \multirow{2}{*}{$\begin{array}{l}\text { Thickness } \mathbf{x} \\
\text { percent } \mathrm{P}_{2} \mathrm{O}_{5} \\
\text { (cumulative) }\end{array}$} \\
\hline & & & & $\mathrm{P}_{2} \mathrm{O}_{5}$ & $\mathrm{Al}_{2} \mathrm{O}_{3}$ & $\mathrm{Fe}_{2} \mathrm{O}_{3}$ & $\begin{array}{l}\text { Loss on } \\
\text { ignition }\end{array}$ & $\begin{array}{c}\text { Acid } \\
\text { insoluble }\end{array}$ & & \\
\hline
\end{tabular}

Phosphatic shale member of Phosphoria formation-top not cored

\begin{tabular}{|c|c|c|c|c|c|c|c|c|c|c|}
\hline $\begin{array}{l}P-187 \\
P-186 \\
P-185 \\
P-184 \\
P-183\end{array}$ & $\begin{array}{l}\text { Mudstone } \\
\text { Mudstone } \\
\text { Mudstone } \\
\text { Mudstone and phosphate rock } \\
\text { Phosphate rock }\end{array}$ & $\begin{array}{l}\text { 801-RMC } \\
\text { 802-RMC } \\
\text { 803-RMC } \\
\text { 804-RMC } \\
\text { 805-RMC }\end{array}$ & $\begin{array}{l}2.3 \\
2.0 \\
3.5 \\
0.4 \\
0.3\end{array}$ & $\begin{array}{r}3.7 \\
5.4 \\
1.5 \\
16.4 \\
33.2\end{array}$ & $\begin{array}{l}-- \\
-- \\
-- \\
--\end{array}$ & $\begin{array}{l}-- \\
-- \\
-- \\
--\end{array}$ & $\begin{array}{l}-- \\
-- \\
-- \\
--\end{array}$ & $\begin{array}{r}72.5 \\
68.0 \\
79.5 \\
38.3 \\
8.9\end{array}$ & $\begin{array}{l}2.3 \\
4.3 \\
7.8 \\
8.2 \\
8.5\end{array}$ & $\begin{array}{r}8.51 \\
19.31 \\
24.56 \\
31.12 \\
41.08\end{array}$ \\
\hline \multirow[t]{2}{*}{$\begin{array}{l}P-182 \\
P-181\end{array}$} & $\begin{array}{l}\text { Mudstone, phosphatic } \\
\text { Phosphate rock }\end{array}$ & $\begin{array}{l}\text { 806-RMC } \\
\text { 807-RMC }\end{array}$ & $\begin{array}{l}0.7 \\
0.6\end{array}$ & 32.5 & -- & -- & -- & 12.4 & $\begin{array}{l}9.2 \\
9.8\end{array}$ & $\begin{array}{l}63.83 \\
83.33\end{array}$ \\
\hline & \multicolumn{9}{|c|}{ The $\mathrm{P}_{2} \mathrm{O}_{5}$ and acid insoluble analyses represent a composite of samples 806-RMC and 807-RMC. } & \\
\hline $\begin{array}{l}P-180 \\
P-179 \\
P-178\end{array}$ & $\begin{array}{l}\text { Mudstone, phosphatic } \\
\text { Phosphate rock, argillaceous } \\
\text { Mudstone, phosphatic }\end{array}$ & $\begin{array}{l}\text { 808-RMC } \\
\text { 809-RMC } \\
\text { 810-RMC }\end{array}$ & $\begin{array}{l}0.6 \\
0.7 \\
0.7\end{array}$ & $\begin{array}{l}16.4 \\
23.1 \\
11.5\end{array}$ & $\begin{array}{ll}-- \\
--\end{array}$ & -- & $\begin{array}{l}-- \\
-- \\
--\end{array}$ & $\begin{array}{l}48.1 \\
33.0 \\
57.6\end{array}$ & $\begin{array}{l}10.4 \\
11.1 \\
11.8\end{array}$ & $\begin{array}{r}93.17 \\
109.34 \\
117.39\end{array}$ \\
\hline \multirow[t]{2}{*}{$\begin{array}{l}P-177 \\
P-176 \\
P-175 \\
P-174 \\
P-173\end{array}$} & $\begin{array}{l}\text { Phosphate rock } \\
\text { Phosphate rock, argillaceous } \\
\text { Core missing } \\
\text { Phosphate rock } \\
\text { Phosphate rock }\end{array}$ & $\begin{array}{l}811-R M C \\
81 z-R M C \\
-- \\
813-\text { RMC } \\
814-\text { RMC }\end{array}$ & $\begin{array}{l}1.0 \\
0.4 \\
0.4 \\
0.4 \\
0.4\end{array}$ & $\begin{array}{c}35.9 \\
26.5 \\
-- \\
23.9\end{array}$ & $\begin{array}{l}0.83 \\
4.2 \\
-.8 \\
4.8 \\
5.4\end{array}$ & $\begin{array}{l}0.33 \\
1.2 \\
-. \\
0.94 \\
1.07\end{array}$ & $\begin{array}{l}3.34 \\
5.38 \\
-- \\
6.64 \\
6.54\end{array}$ & $\begin{array}{r}5.5 \\
22.0 \\
-- \\
28.1\end{array}$ & $\begin{array}{l}12.8 \\
13.2 \\
13.6 \\
14.0 \\
14.4\end{array}$ & $\begin{array}{c}153.29 \\
163.89 \\
\begin{array}{c}9.56 * \\
19.12\end{array}\end{array}$ \\
\hline & \multicolumn{9}{|c|}{ The $\mathrm{P}_{2} \mathrm{O}_{5}$ and acid insoluble analyses represent a composite of samples 813-RMC and 814-RMC. } & \\
\hline $\begin{array}{l}P-172 \\
P-171 \\
P-170 \\
P-169 \\
P-168\end{array}$ & $\begin{array}{l}\text { Phosphate rock, argillaceous } \\
\text { Core missing } \\
\text { Phosphate rock } \\
\text { Core missing } \\
\text { Phosphate rock }\end{array}$ & $\begin{array}{l}815-\mathrm{RMC} \\
816-\mathrm{RMC} \\
817-\mathrm{RMC}\end{array}$ & $\begin{array}{l}0.8 \\
0.1 \\
0.9 \\
0.1 \\
0.9\end{array}$ & $\begin{array}{c}26.5 \\
-- \\
34.2 \\
-- \\
27.4\end{array}$ & $\begin{array}{r}2.4 \\
-- \\
1.3 \\
-- \\
2.9\end{array}$ & $\begin{array}{l}0.71 \\
-- \\
0.32 \\
-- \\
0.68\end{array}$ & $\begin{array}{c}7.12 \\
-- \\
5.00 \\
-- \\
7.72\end{array}$ & $\begin{array}{c}22.7 \\
-- \\
7.7 \\
-- \\
19.1\end{array}$ & $\begin{array}{l}15.2 \\
15.3 \\
16.2 \\
16.3 \\
17.2\end{array}$ & 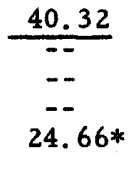 \\
\hline $\begin{array}{l}P-167 \\
P-166\end{array}$ & $\begin{array}{l}\text { Phosphate rock, argillaceous } \\
\text { Phosphate rock and phosphatic } \\
\text { mudstone }\end{array}$ & $\begin{array}{l}818-\mathrm{RMC} \\
819-\mathrm{RMC}\end{array}$ & $\begin{array}{l}0.5 \\
0.9\end{array}$ & $\begin{array}{l}23.9 \\
31.6\end{array}$ & $\begin{array}{r}5.3 \\
2.1\end{array}$ & $\begin{array}{l}0.46 \\
0.37\end{array}$ & $\begin{array}{l}8.44 \\
8.40\end{array}$ & $\begin{array}{r}28.2 \\
8.7\end{array}$ & $\begin{array}{l}17.7 \\
18.6\end{array}$ & $\begin{array}{l}36.61 \\
68.21\end{array}$ \\
\hline $\begin{array}{l}P-165 \\
P-164\end{array}$ & $\begin{array}{l}\text { Core missing } \\
\text { Phosphate rock }\end{array}$ & $820-\mathrm{RMC}$ & $\begin{array}{l}0.1 \\
1.0\end{array}$ & 34.8 & $\overline{1.1}$ & $0 . \overline{39}$ & $8 . \overline{60}$ & $\overline{1.5}$ & $\begin{array}{l}18.7 \\
19.7\end{array}$ & -- \\
\hline
\end{tabular}

* Cumulative data incomplete due to missing information. Computations start from zero after interruption. 


\begin{tabular}{|c|c|}
\hline $\begin{array}{l}P-163 \\
P-162 \\
P-161\end{array}$ & $\begin{array}{l}\text { Core missing } \\
\text { Phosphate rock } \\
\text { Phosphate rock and phosphatic } \\
\text { mudstone }\end{array}$ \\
\hline $\begin{array}{l}P-160 \\
P-159\end{array}$ & $\begin{array}{l}\text { Phosphate rock, argillaceous } \\
\text { Mudstone, phosphatic }\end{array}$ \\
\hline $\begin{array}{l}P-158 \\
P-157 \\
P-156 \\
P-155 \\
P-154\end{array}$ & $\begin{array}{l}\text { Phosphate rock } \\
\text { Mudstone } \\
\text { Mudstone } \\
\text { Mudstone } \\
\text { Mudstone }\end{array}$ \\
\hline $\begin{array}{l}P-153 \\
P-152 \\
P-151 \\
P-150 \\
P-149\end{array}$ & $\begin{array}{l}\text { Mudstone } \\
\text { Mudstone } \\
\text { Mudstone } \\
\text { Mudstone } \\
\text { Mudstone }\end{array}$ \\
\hline $\begin{array}{l}P-148 \\
P-147 \\
P-146 \\
P-145 \\
P-144\end{array}$ & $\begin{array}{l}\text { Phosphate rock, calcareous } \\
\text { Phosphate rock, calcareous } \\
\text { Phosphate rock } \\
\text { Phosphate rock } \\
\text { Phosphate rock }\end{array}$ \\
\hline $\begin{array}{l}P-143 \\
P-142 \\
P-141 \\
P-140 \\
P-139\end{array}$ & $\begin{array}{l}\text { Phosphate rock, argillaceous } \\
\text { Phosphate rock, argillaceous } \\
\text { Phosphate rock, argillaceous } \\
\text { Phosphate rock, argillaceous } \\
\text { Core missing }\end{array}$ \\
\hline $\begin{array}{l}P-138 \\
P-137 \\
P-136 \\
P-135 \\
P-134\end{array}$ & $\begin{array}{l}\text { Phosphate rock, argillaceous } \\
\text { Phosphate rock, argillaceous } \\
\text { Phosphate rock and mudstone } \\
\text { Phosphate rock, argillaceous } \\
\text { Mudstone, phosphatic }\end{array}$ \\
\hline $\begin{array}{l}P-133 \\
P-132 \\
P-131 \\
P-130 \\
P-129\end{array}$ & $\begin{array}{l}\text { Mudstone, phosphatic } \\
\text { Phosphate rock, argillaceous } \\
\text { Mudstone, phosphatic } \\
\text { Mudstone } \\
\text { Mudstorie }\end{array}$ \\
\hline $\begin{array}{l}P-128 \\
P=127 \\
P-126 \\
P-125\end{array}$ & $\begin{array}{l}\text { Mudstone, phosphatic } \\
\text { Mudstone, phosphatic } \\
\text { Mudstone, phosphatic } \\
\text { Mudstone, phosphatic }\end{array}$ \\
\hline
\end{tabular}

\begin{tabular}{|c|}
\hline $821-\overline{R M C}$ \\
\hline $\begin{array}{l}\text { 822-RMC } \\
823-R M C \\
824-R M C\end{array}$ \\
\hline $\begin{array}{l}\text { 825-RMC } \\
\text { 826-RMC } \\
\text { 827-RMC } \\
828-R M C \\
\text { 829-RMC }\end{array}$ \\
\hline $\begin{array}{l}830-\text { RMC } \\
831-\text { RLP } \\
832-\text { RLP } \\
833-\text { RLP } \\
834-\text { RLP }\end{array}$ \\
\hline $\begin{array}{l}835-\text { RLP } \\
836-\text { JES } \\
837-\text { DAB } \\
838-\text { DAB } \\
839-\text { DAB }\end{array}$ \\
\hline $\begin{array}{c}840-\text { DAB } \\
841-\text { DAB } \\
842-\text { DAB } \\
843-\text { DAB } \\
\ldots\end{array}$ \\
\hline $\begin{array}{l}\text { 844- DAB } \\
\text { 845-RMC } \\
\text { 846-RMC } \\
\text { 847-RMC } \\
\text { 848-RAH }\end{array}$ \\
\hline $\begin{array}{l}\text { 849- RAH } \\
\text { 850- RAH } \\
\text { 851-RAH } \\
\text { 852- RAH } \\
\text { 853- RAH }\end{array}$ \\
\hline $\begin{array}{l}\text { 854-RAH } \\
\text { 855-RAH } \\
\text { 856-RAH } \\
\text { 857-RAH }\end{array}$ \\
\hline
\end{tabular}

\begin{tabular}{|c|c|c|c|c|c|}
\hline $\begin{array}{l}0.4 \\
1.0\end{array}$ & 25.3 & $3 .-$ & 0.64 & $12 . \overline{40}$ & 19.1 \\
\hline $\begin{array}{l}0.6 \\
1.0 \\
1.0\end{array}$ & $\begin{array}{l}31.4 \\
23.9 \\
15.9\end{array}$ & $\begin{array}{c}2.2 \\
3.7 \\
--\end{array}$ & $\begin{array}{l}0.57 \\
1.1 \\
-.-\end{array}$ & $\begin{array}{r}8.08 \\
13.84 \\
--\end{array}$ & $\begin{array}{r}9.6 \\
20.5 \\
41.5\end{array}$ \\
\hline $\begin{array}{l}0.4 \\
0.4 \\
0.6 \\
0.3 \\
0.4\end{array}$ & $\begin{array}{r}21.7 \\
6.4 \\
4.5 \\
7.2 \\
2.2\end{array}$ & $\begin{array}{l}-- \\
-- \\
-- \\
--\end{array}$ & $\begin{array}{l}-- \\
-- \\
-- \\
--\end{array}$ & $\begin{array}{l}-- \\
-- \\
-- \\
--\end{array}$ & $\begin{array}{r}7.8 \\
55.8 \\
54.7 \\
45.6 \\
58.9\end{array}$ \\
\hline $\begin{array}{l}0.4 \\
0.9 \\
0.6 \\
0.4 \\
1.1\end{array}$ & $\begin{array}{l}1.6 \\
0.8 \\
2.5 \\
6.1 \\
2.0\end{array}$ & $\begin{array}{l}-- \\
-- \\
-- \\
--\end{array}$ & $\begin{array}{l}- \\
-- \\
-- \\
--\end{array}$ & $\begin{array}{l}-- \\
-- \\
-- \\
-- \\
--\end{array}$ & $\begin{array}{l}60.8 \\
62.3 \\
55.3 \\
50.6 \\
57.0\end{array}$ \\
\hline $\begin{array}{l}0.5 \\
0.7 \\
0.9 \\
0.5 \\
0.5\end{array}$ & $\begin{array}{l}16.6 \\
22.1 \\
26.7 \\
34.8 \\
29.6\end{array}$ & $\begin{array}{l}-- \\
-- \\
-- \\
--\end{array}$ & $\begin{array}{l}-- \\
-- \\
-- \\
--\end{array}$ & $\begin{array}{l}-- \\
-- \\
-- \\
--\end{array}$ & $\begin{array}{r}19.5 \\
19.1 \\
15.8 \\
5.6 \\
5.6\end{array}$ \\
\hline $\begin{array}{l}1.5 \\
0.8 \\
0.6 \\
0.9 \\
1.9\end{array}$ & $\begin{array}{c}14.9 \\
13.8 \\
17.1 \\
16.9 \\
--\end{array}$ & $\begin{array}{l}-- \\
-- \\
-- \\
--\end{array}$ & $\begin{array}{l}-- \\
-- \\
-- \\
--\end{array}$ & $\begin{array}{l}-- \\
-- \\
-- \\
--\end{array}$ & $\begin{array}{c}17.5 \\
17.4 \\
23.1 \\
17.9 \\
--\end{array}$ \\
\hline $\begin{array}{l}1.8 \\
1.0 \\
1.0 \\
1.0 \\
2.4\end{array}$ & $\begin{array}{r}16.6 \\
17.1 \\
16.7 \\
16.4 \\
7.8\end{array}$ & $\begin{array}{l}-- \\
-- \\
-- \\
-- \\
--\end{array}$ & $\begin{array}{l}-- \\
-- \\
-- \\
-- \\
--\end{array}$ & $\begin{array}{l}-- \\
-- \\
-- \\
-- \\
-\infty\end{array}$ & $\begin{array}{l}32.6 \\
27.0 \\
31.1 \\
33.8 \\
61.1\end{array}$ \\
\hline $\begin{array}{l}0.4 \\
0.4 \\
0.9 \\
1.1 \\
1.8\end{array}$ & $\begin{array}{r}14.9 \\
16.5 \\
12.7 \\
6.6 \\
5.5\end{array}$ & $\begin{array}{l}-- \\
-- \\
-- \\
--\end{array}$ & $\begin{array}{l}-- \\
-- \\
-- \\
-- \\
--\end{array}$ & $\begin{array}{l}-- \\
-- \\
-- \\
--\end{array}$ & $\begin{array}{l}39.9 \\
35.8 \\
49.0 \\
63.8 \\
66.9\end{array}$ \\
\hline $\begin{array}{l}1.0 \\
0.9 \\
1.1 \\
1.1\end{array}$ & $\begin{array}{r}11.6 \\
8.6 \\
9.7 \\
9.7\end{array}$ & $\begin{array}{l}-- \\
-- \\
--\end{array}$ & $\begin{array}{l}-- \\
-- \\
--\end{array}$ & $\begin{array}{l}-- \\
-- \\
-- \\
-\infty\end{array}$ & $\begin{array}{l}52.6 \\
68.0 \\
67.6 \\
60.9\end{array}$ \\
\hline
\end{tabular}

\begin{tabular}{|c|c|}
\hline $\begin{array}{l}20.1 \\
21.1\end{array}$ & $25.30 *$ \\
\hline $\begin{array}{l}21.7 \\
22.7 \\
23.7\end{array}$ & $\begin{array}{l}44.14 \\
68.04 \\
83.94\end{array}$ \\
\hline $\begin{array}{l}24.1 \\
24.5 \\
25.1 \\
25.4 \\
25.8\end{array}$ & $\begin{array}{r}92.62 \\
95.18 \\
97.88 \\
100.04 \\
100.92\end{array}$ \\
\hline $\begin{array}{l}26.2 \\
27.1 \\
27.7 \\
28.1 \\
29.2\end{array}$ & $\begin{array}{l}101.56 \\
102.28 \\
103.78 \\
106.22 \\
108.42\end{array}$ \\
\hline $\begin{array}{l}29.7 \\
30.4 \\
31.3 \\
31.8 \\
32.3\end{array}$ & $\begin{array}{l}116.72 \\
132.19 \\
156.22 \\
173.62 \\
188.42\end{array}$ \\
\hline $\begin{array}{l}33.8 \\
34.6 \\
35.2 \\
36.1 \\
38.0\end{array}$ & $\begin{array}{r}210.77 \\
221.81 \\
232.07 \\
247.28 \\
--\end{array}$ \\
\hline $\begin{array}{l}39.8 \\
40.8 \\
41.8 \\
42.8 \\
45.2\end{array}$ & $\begin{array}{l}29.88 * \\
46.98 \\
63.68 \\
80.08 \\
98.80\end{array}$ \\
\hline $\begin{array}{l}45.6 \\
46.0 \\
46.9 \\
48.0 \\
49.8\end{array}$ & $\begin{array}{l}104.76 \\
111.36 \\
122.79 \\
130.05 \\
139.95\end{array}$ \\
\hline $\begin{array}{l}50.8 \\
51.7 \\
52.8 \\
53.9\end{array}$ & $\begin{array}{l}151.55 \\
159.29 \\
169.96 \\
180.63 \\
\end{array}$ \\
\hline
\end{tabular}

* Cumulative data incomplete due to missing information. Computations start from zero after interruption.

* Cumulative data in 


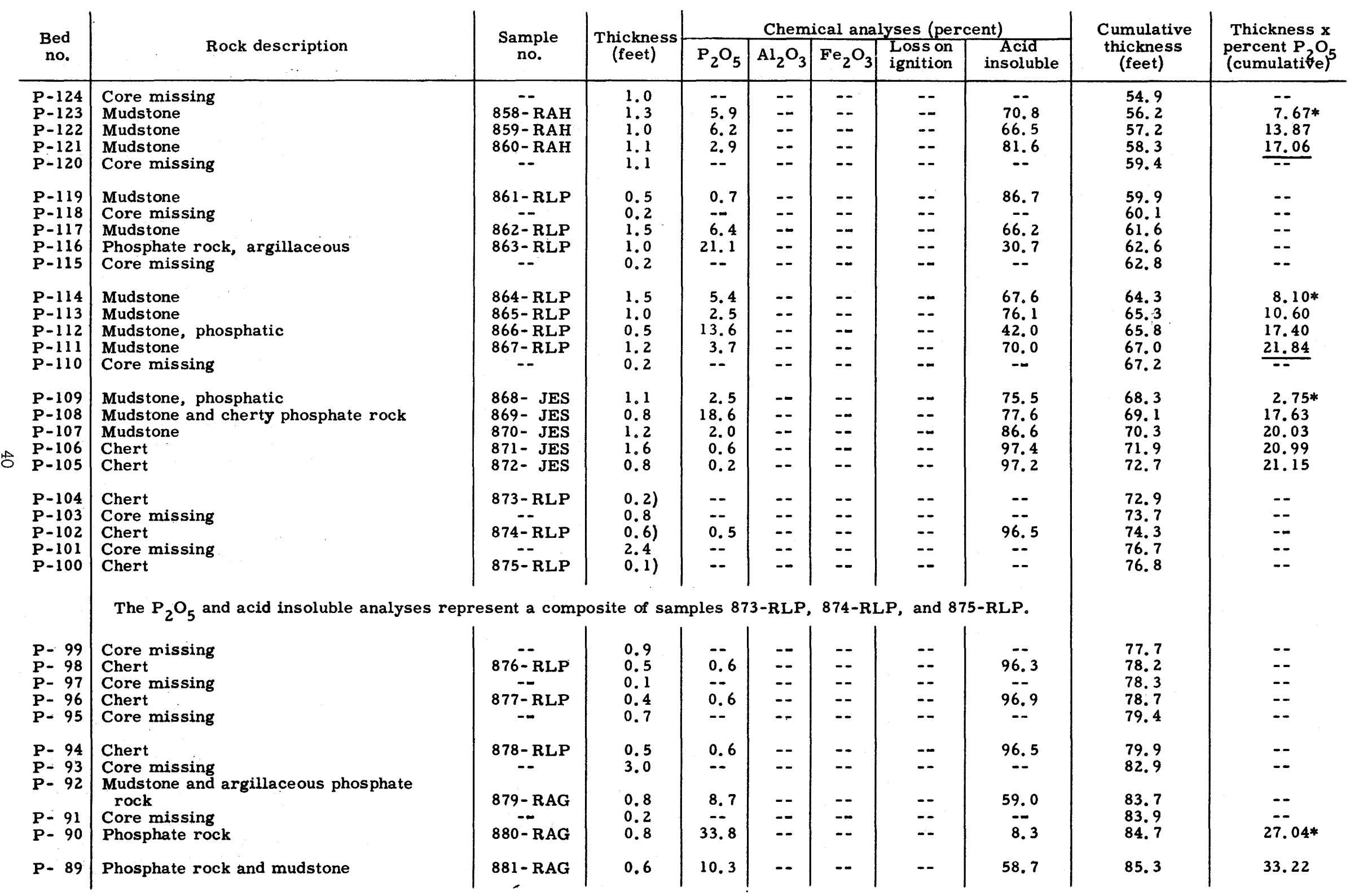




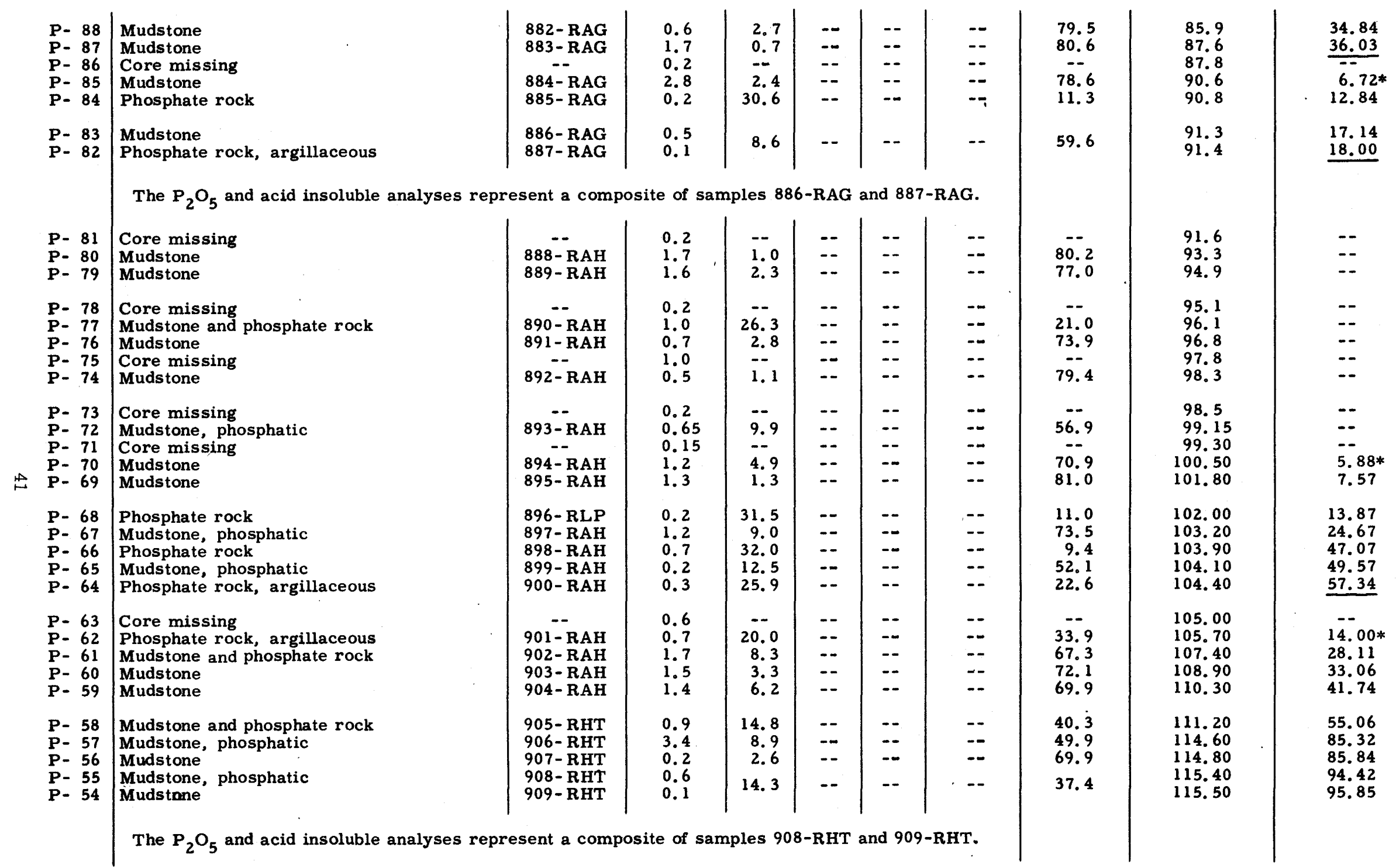

* Cumulative data incomplete due to missing information. Computations start from zero after interruption. 


\begin{tabular}{|c|c|c|c|c|c|c|c|c|c|c|}
\hline \multirow{2}{*}{$\begin{array}{c}\text { Bed } \\
\text { no. }\end{array}$} & \multirow[b]{2}{*}{ Rock description } & \multirow{2}{*}{$\begin{array}{c}\text { Sample } \\
\text { no. }\end{array}$} & \multirow{2}{*}{$\begin{array}{c}\text { Thickness } \\
\text { (feet) }\end{array}$} & \multicolumn{5}{|c|}{ Chemical analyses (percent) } & \multirow{2}{*}{$\begin{array}{l}\text { Cumulative } \\
\text { thickness. } \\
\text { (feet) }\end{array}$} & \multirow{2}{*}{$\begin{array}{l}\text { Thickness } \mathrm{x} \\
\text { percent } \mathrm{P}_{2} \mathrm{O}_{5} \\
\text { (cumulative) }\end{array}$} \\
\hline & & & & $\mathrm{P}_{2} \mathrm{O}_{5}$ & $\mathrm{Al}_{2} \mathrm{O}_{3}$ & $\mathrm{Fe}_{2} \mathrm{O}_{3}$ & $\begin{array}{l}\text { Loss on } \\
\text { ignition }\end{array}$ & $\begin{array}{c}\text { Acid } \\
\text { insoluble }\end{array}$ & & \\
\hline $\begin{array}{l}P-53 \\
P-52 \\
P-51 \\
P=50 \\
P=49\end{array}$ & $\begin{array}{l}\text { Mudstone, phosphatic } \\
\text { Mudstone, phosphatic } \\
\text { Core missing } \\
\text { Mudstone, phosphatic } \\
\text { Mudstone }\end{array}$ & $\begin{array}{l}\text { 910- RHT } \\
\text { 911-RHT } \\
-- \\
\text { 912-RHT } \\
\text { 913-RHT }\end{array}$ & $\begin{array}{l}1.4 \\
0.2 \\
0.5 \\
0.5 \\
0.8\end{array}$ & $\begin{array}{l}12.4 \\
8.5 \\
-- \\
9.4 \\
7.4\end{array}$ & $\begin{array}{l}-- \\
-- \\
-- \\
--\end{array}$ & $\begin{array}{l}-- \\
-- \\
-- \\
--\end{array}$ & $\begin{array}{l}-- \\
-- \\
-- \\
--\end{array}$ & $\begin{array}{l}37.3 \\
46.3 \\
-- \\
52.6 \\
50.2\end{array}$ & $\begin{array}{l}116.90 \\
117.10 \\
117.60 \\
118.10 \\
118.90\end{array}$ & $\begin{array}{l}113.21 \\
\frac{114.91}{--} \\
4.70 * \\
10.62\end{array}$ \\
\hline $\begin{array}{l}P-48 \\
P-47 \\
P-46 \\
P-45 \\
P-44\end{array}$ & $\begin{array}{l}\text { Mudstone, phosphatic } \\
\text { Mudstone, phosphatic } \\
\text { Mudstone, phosphatic } \\
\text { Mudstone } \\
\text { Mudstone }\end{array}$ & $\begin{array}{l}\text { 914- RHT } \\
\text { 915- RHT } \\
916-\text { RHT } \\
917-\text { RHT } \\
918-\text { RHT }\end{array}$ & $\begin{array}{l}0.8 \\
0.6 \\
0.8 \\
1.4 \\
0.5\end{array}$ & $\begin{array}{l}9.3 \\
8.6 \\
8.6 \\
4.3 \\
0.9\end{array}$ & $\begin{array}{l}-- \\
-- \\
-- \\
--\end{array}$ & $\begin{array}{l}-- \\
-- \\
-- \\
--\end{array}$ & $\begin{array}{l}-- \\
-- \\
-- \\
--\end{array}$ & $\begin{array}{l}48.8 \\
49.9 \\
57.7 \\
62.6 \\
71.5\end{array}$ & $\begin{array}{l}119.70 \\
120.30 \\
121.10 \\
122.50 \\
123.00\end{array}$ & $\begin{array}{l}18.06 \\
23.22 \\
30.10 \\
36.12 \\
36.57\end{array}$ \\
\hline $\begin{array}{l}P-43 \\
P-42 \\
P-41 \\
P-40 \\
P-39\end{array}$ & $\begin{array}{l}\text { Mudstone } \\
\text { Mudstone } \\
\text { Mudstone } \\
\text { Mudstone } \\
\text { Mudstone }\end{array}$ & $\begin{array}{l}919-\text { RHT } \\
920-\text { RHT } \\
921-\text { RHT } \\
922-\text { RHT } \\
923-\text { RHT }\end{array}$ & $\begin{array}{l}0.2 \\
1.6 \\
1.2 \\
0.2 \\
0.4\end{array}$ & $\begin{array}{l}1.4 \\
5.4 \\
3.3 \\
3.9 \\
6.6\end{array}$ & $\begin{array}{l}-- \\
-- \\
-- \\
-- \\
--\end{array}$ & $\begin{array}{l}-- \\
-- \\
-- \\
--\end{array}$ & $\begin{array}{l}-- \\
-- \\
-- \\
--\end{array}$ & $\begin{array}{l}71.2 \\
60.8 \\
65.6 \\
68.8 \\
57.7\end{array}$ & $\begin{array}{l}123.20 \\
124.80 \\
126.00 \\
126.20 \\
126.60\end{array}$ & $\begin{array}{l}36.85 \\
45.49 \\
49.45 \\
50.23 \\
52.87\end{array}$ \\
\hline $\begin{array}{l}P-38 \\
P-37 \\
P-36 \\
P-35 \\
P-34\end{array}$ & $\begin{array}{l}\text { Mudstone } \\
\text { Mudstone } \\
\text { Mudstone, phosphatic, calcareous } \\
\text { Mudstone, phosphatic } \\
\text { Mudstone, phosphatic }\end{array}$ & $\begin{array}{l}924-\text { RHT } \\
925-\text { RHT } \\
926-\text { DFD } \\
927-\text { DFD } \\
928-\text { DFD }\end{array}$ & $\begin{array}{l}0.2 \\
2.0 \\
2.5 \\
2.0 \\
1.2\end{array}$ & $\begin{array}{r}6.8 \\
3.0 \\
9.8 \\
9.6 \\
12.3\end{array}$ & $\begin{array}{l}-- \\
-- \\
-- \\
--\end{array}$ & $\begin{array}{l}-- \\
-- \\
-- \\
--\end{array}$ & $\begin{array}{l}-- \\
-- \\
-- \\
--\end{array}$ & $\begin{array}{l}65.2 \\
68.6 \\
49.3 \\
49.7 \\
44.3\end{array}$ & $\begin{array}{l}126.80 \\
128.80 \\
131.30 \\
133.30 \\
134.50\end{array}$ & $\begin{array}{r}54.23 \\
60.23 \\
84.73 \\
103.93 \\
118.69 \\
\end{array}$ \\
\hline $\begin{array}{l}P-33 \\
P-32\end{array}$ & $\begin{array}{l}\text { Core missing } \\
\text { Mudstone, phosphatic, and calcareous } \\
\text { mudstone }\end{array}$ & $929-$ DFD & $\begin{array}{l}0.4 \\
3.3\end{array}$ & 10.6 & -- & -- & -- & -- & 134.90 & $34.98 *$ \\
\hline $\begin{array}{l}P-31 \\
P=30 \\
P-29\end{array}$ & $\begin{array}{l}\text { Mudstone, phosphatic, calcareous } \\
\text { Mudstone } \\
\text { Mudstone }\end{array}$ & $\begin{array}{l}930-\text { DFD } \\
931-\text { DFD } \\
932-\text { DFD }\end{array}$ & $\begin{array}{l}3.3 \\
1.2 \\
2.8 \\
1.5\end{array}$ & $\begin{array}{r}10.0 \\
10.9 \\
7.2 \\
7.5\end{array}$ & -- & -- & -- & $\begin{array}{l}41.0 \\
51.7 \\
58.0 \\
51.0\end{array}$ & $\begin{array}{l}139.40 \\
142.20 \\
143.70\end{array}$ & $\begin{array}{l}34.730 \\
48.06 \\
68.22 \\
79.47\end{array}$ \\
\hline $\begin{array}{l}P-28 \\
P-27 \\
P-26 \\
P-25 \\
P-24\end{array}$ & $\begin{array}{l}\text { Mudstone, calcareous, phosphatic } \\
\text { Core missing } \\
\text { Mudstone, phosphatic } \\
\text { Mudstone, phosphatic } \\
\text { Mudstone }\end{array}$ & $\begin{array}{l}933-\text { DFD } \\
934-\text { DFD } \\
935-\text { DFD } \\
936-\text { RHT }\end{array}$ & $\begin{array}{l}0.9 \\
1.5 \\
0.7 \\
2.2 \\
0.6\end{array}$ & $\begin{array}{r}9.8 \\
-- \\
8.1 \\
13.4 \\
5.6\end{array}$ & $\begin{array}{l}-- \\
-- \\
-- \\
--\end{array}$ & $\begin{array}{l}-- \\
-- \\
-- \\
--\end{array}$ & $\begin{array}{l}-- \\
-- \\
-- \\
--\end{array}$ & $\begin{array}{l}46.0 \\
-- \\
61.8 \\
39.7 \\
69.7\end{array}$ & $\begin{array}{l}144.60 \\
146.10 \\
146.80 \\
149.00 \\
149.60\end{array}$ & $\begin{array}{l}\frac{88.29}{--} \\
5.67^{*} \\
35.15 \\
38.51\end{array}$ \\
\hline $\begin{array}{l}P-23 \\
P-22 \\
P-21 \\
P-20 \\
P-19\end{array}$ & $\begin{array}{l}\text { Mudstone, phosphatic, calcareous } \\
\text { Mudstone, phosphatic } \\
\text { Mudstone, phosphatic } \\
\text { Phosphate rock, argillaceous } \\
\text { Mudstone, phosphatic }\end{array}$ & $\begin{array}{l}\text { 937- RHT } \\
\text { 938- RHT } \\
939-\text { RHT } \\
\text { 940- RHT } \\
941-\text { RHT }\end{array}$ & $\begin{array}{l}0.8 \\
1.0 \\
0.4 \\
3.5 \\
0.3\end{array}$ & $\begin{array}{l}11.7 \\
10.6 \\
12.0 \\
17.9 \\
14.7\end{array}$ & $\begin{array}{l}-- \\
-- \\
-- \\
--\end{array}$ & $\begin{array}{l}-- \\
-- \\
-- \\
--\end{array}$ & $\begin{array}{l}-- \\
-- \\
-- \\
--\end{array}$ & $\begin{array}{l}47.0 \\
46.5 \\
51.3 \\
30.4 \\
43.7\end{array}$ & $\begin{array}{l}150.40 \\
151.40 \\
151.80 \\
155.30 \\
155.60\end{array}$ & $\begin{array}{r}47.87 \\
58.47 \\
63.27 \\
125.92 \\
130.33\end{array}$ \\
\hline $\begin{array}{l}P-18 \\
P-17 \\
P-16 \\
P-15 \\
P-14\end{array}$ & $\begin{array}{l}\text { Phosphate rock, argillaceous } \\
\text { Phosphate rock and mudstone } \\
\text { Mudstone, phosphatic } \\
\text { Mudstone, phosphatic } \\
\text { Mudstone, phosphatic }\end{array}$ & $\begin{array}{l}\text { 942- RHT } \\
\text { 943- RHT } \\
\text { 944- RHT } \\
\text { 945-RHT } \\
\text { 946-RHT }\end{array}$ & $\begin{array}{l}2.0 \\
2.6 \\
0.6 \\
1.9 \\
6.0\end{array}$ & $\begin{array}{r}18.3 \\
19.4 \\
16.6 \\
10.6 \\
9.1\end{array}$ & $\begin{array}{l}-- \\
-- \\
-- \\
--\end{array}$ & $\begin{array}{l}-- \\
-- \\
-- \\
--\end{array}$ & $\begin{array}{l}-- \\
-- \\
-- \\
-- \\
--\end{array}$ & $\begin{array}{l}26.7 \\
37.8 \\
46.1 \\
59.4 \\
63.2\end{array}$ & $\begin{array}{l}157.60 \\
160.20 \\
160.80 \\
162.70 \\
168.70\end{array}$ & $\begin{array}{l}166.93 \\
217.37 \\
227.33 \\
247.47 \\
302.07\end{array}$ \\
\hline
\end{tabular}




\begin{tabular}{|c|c|c|c|c|c|c|c|c|c|c|}
\hline $\begin{array}{l}P-13 \\
P-12 \\
P=11 \\
P=10 \\
P=9\end{array}$ & $\begin{array}{l}\text { Phosphate rock, argillaceous } \\
\text { Phosphate rock and mudstone } \\
\text { Mudstone } \\
\text { Phosphate rock, argillaceous } \\
\text { Mudstone, phosphatic }\end{array}$ & $\begin{array}{l}\text { 947- RHT } \\
\text { 948- RHT } \\
\text { 949- RHT } \\
\text { 950- RHT } \\
\text { 951- RHT }\end{array}$ & $\begin{array}{l}1.2 \\
2.0 \\
0.7 \\
1.2 \\
0.9\end{array}$ & $\begin{array}{r}27.4 \\
23.3 \\
6.2 \\
17.9 \\
8.6\end{array}$ & $\begin{array}{l}2.2 \\
4.9 \\
9.3 \\
6.6 \\
9.8\end{array}$ & $\begin{array}{l}0.55 \\
1.4 \\
3.2 \\
1.5 \\
2.6\end{array}$ & $\begin{array}{l}3.98 \\
4.20 \\
4.62 \\
4.44 \\
3.84\end{array}$ & $\begin{array}{l}24.8 \\
30.7 \\
69.0 \\
43.3 \\
33.4\end{array}$ & $\begin{array}{l}169.90 \\
171.90 \\
172.60 \\
173.80 \\
174.70\end{array}$ & $\begin{array}{l}334.95 \\
381.55 \\
385.89 \\
407.39 \\
415.13\end{array}$ \\
\hline $\begin{array}{ll}P- & 8 \\
P- & 7 \\
P- & 6 \\
P- & 5 \\
P- & 4\end{array}$ & $\begin{array}{l}\text { Mudstone and phosphate rock } \\
\text { Core missing } \\
\text { Mudstone, phosphatic } \\
\text { Phosphate rock, argillaceous } \\
\text { Core missing }\end{array}$ & $\begin{array}{l}952-\text { RHT } \\
\text { 953-- RHT } \\
954-\text { RHT } \\
--\end{array}$ & $\begin{array}{l}5.4 \\
1.4 \\
0.7 \\
0.5 \\
5.0\end{array}$ & $\begin{array}{l}21.1 \\
-- \\
12.3 \\
20.5 \\
--\end{array}$ & $\begin{array}{c}4.6 \\
-- \\
9.8 \\
5.6 \\
--\end{array}$ & $\begin{array}{r}1.3 \\
-- \\
3.2 \\
1.3 \\
--\end{array}$ & $\begin{array}{l}3.90 \\
-- \\
4.58 \\
4.60 \\
--\end{array}$ & $\begin{array}{l}37.3 \\
-- \\
52.9 \\
34.9 \\
--\end{array}$ & $\begin{array}{l}180.10 \\
181.50 \\
182.20 \\
182.70 \\
187.79\end{array}$ & $\frac{529.07}{--}$ \\
\hline $\begin{array}{l}P-3 \\
P- \\
P=\end{array}$ & $\begin{array}{l}\text { Mudstone } \\
\text { Limestone breccia, argillaceous } \\
\text { Phosphate rock and chert }\end{array}$ & $\begin{array}{l}\text { 955- RHT } \\
956-\text { RHT } \\
\text { 957- RHT }\end{array}$ & $\begin{array}{l}7.0 \\
0.5 \\
5.9\end{array}$ & $\begin{array}{r}5.8 \\
1.3 \\
24.9 \\
\end{array}$ & $\begin{array}{l}-- \\
--\end{array}$ & -- & -- & $\begin{array}{l}68.4 \\
41.5 \\
27.3\end{array}$ & $\begin{array}{l}194.70 \\
195.20 \\
201.10\end{array}$ & $\begin{array}{l}40.60 * \\
41.25 \\
188.16 * *\end{array}$ \\
\hline \multicolumn{11}{|c|}{ Wells formation - top beds only } \\
\hline $\begin{array}{l}C w-1 \\
C w-2 \\
C w-3\end{array}$ & $\begin{array}{l}\text { Limestone, argillaceous } \\
\text { Limestone and sandstone } \\
\text { Limestone breccia, cherty }\end{array}$ & $\begin{array}{l}\text { 958- RHT } \\
959-\text { RHT } \\
960-\text { RHT }\end{array}$ & $\begin{array}{l}1.0 \\
6.7 \\
1.6\end{array}$ & $\begin{array}{l}0.4 \\
1.4 \\
0.8\end{array}$ & $\begin{array}{l}-- \\
--\end{array}$ & $\begin{array}{l}-- \\
--\end{array}$ & $\begin{array}{l}-- \\
--\end{array}$ & $\begin{array}{l}37.3 \\
53.9 \\
68.3\end{array}$ & $\begin{array}{l}1.0 \\
7.7 \\
9.3\end{array}$ & $\begin{array}{r}0.40 \\
9.78 \\
11.06\end{array}$ \\
\hline
\end{tabular}

* Cumulative data incomplete due to missing information. Computations start from zero after interruption.

* Note incompleteness of cumulative data. 
